\title{
European Hernia Society guidelines on the treatment of inguinal hernia in adult patients
}

M. P. Simons · T. Aufenacker · M. Bay-Nielsen · J. L. Bouillot •

G. Campanelli $\cdot$ J. Conze $\cdot$ D. de Lange $\cdot$ R. Fortelny $\cdot$ T. Heikkinen $\cdot$

A. Kingsnorth $\cdot$ J. Kukleta $\cdot$ S. Morales-Conde $\cdot$ P. Nordin $\cdot$ V. Schumpelick $\cdot$

S. Smedberg $\cdot$ M. Smietanski $\cdot$ G. Weber $\cdot$ M. Miserez

Received: 17 June 2009/Accepted: 19 June 2009/Published online: 28 July 2009

(C) The Author(s) 2009. This article is published with open access at Springerlink.com

\author{
Summary \\ Flow diagram \\ General \\ Introduction \\ Motivation \\ Objective \\ Definition \\ Target population \\ Description of problem and initial questions \\ Inguinal hernia treatment for adults in Europe in 2007 \\ Transparency of the process and method \\ Steering and Working Group members \\ Owner and legal significance \\ Intended (target) users \\ Collection and assessment of literature \\ Description of implementation trajectory \\ Procedure for authorising guidelines within the European HerniaSociety \\ Applicability and costs \\ Expiry date \\ Validation \\ Guidelines \\ Indications for treatment \\ Diagnostics \\ Differential diagnosis \\ Classification \\ Risk factors and prevention \\ Treatment of inguinal hernia \\ Inguinal hernia in women \\ Lateral inguinal hernia in young men (18-30 years) \\ Biomaterials
}


Day surgery

Antibiotic prophylaxis

Training aspects

Anaesthesia

Postoperative recovery

Aftercare

Postoperative pain control

Complications

Costs

Questions for the future

Summary general practitioner

Appendix

Definitions and abbreviations

Registration form

Operation techniques

Protocol for local anesthesia

Patient information

AGREE result

Reference list

Abstract The European Hernia Society (EHS) is proud to present the EHS Guidelines for the Treatment of Inguinal Hernia in Adult Patients. The Guidelines contain recommendations for the treatment of inguinal hernia from diagnosis till aftercare. They have been developed by a

This publication can be ordered via http://www.herniaweb.org.

M. P. Simons $(\square)$

Department of Surgery, Onze Lieve Vrouwe Gasthuis Hospital,

Postbus 95500, 1090 HM Amsterdam, The Netherlands

e-mail: mpsimons@telfort.nl; m.p.simons@olvg.nl

T. Aufenacker

Rijnstate Hospital, Arnhem, The Netherlands

M. Bay-Nielsen

Hvidovre University Hospital, Copenhagen, Denmark

J. L. Bouillot

University of Descartes, Paris, France

G. Campanelli

University of Insubria, Milan, Italy

J. Conze · V. Schumpelick

Aachen University, Aachen, Germany

D. de Lange

Westfries Gasthuis, Hoorn, The Netherlands

R. Fortelny

Wilhelminenspital, Vienna, Austria

T. Heikkinen

Oulu University Hospital, Oulu, Finland
Working Group consisting of expert surgeons with representatives of 14 country members of the EHS. They are evidence-based and, when necessary, a consensus was reached among all members. The Guidelines have been reviewed by a Steering Committee. Before finalisation,

\author{
A. Kingsnorth \\ Derriford Hospital, Plymouth, England \\ J. Kukleta \\ Klinik Im Park, Zurich, Switzerland \\ S. Morales-Conde \\ University of Sevilla, Seville, Spain \\ P. Nordin \\ Östersund Hospital, Ostersund, Sweden \\ S. Smedberg \\ Helsingborg Hospital, Helsingborg, Sweden \\ M. Smietanski \\ Medical University of Gdansk, Gdansk, Poland \\ G. Weber \\ Medical Faculty, University of Pécs, Pecs, Hungary \\ M. Miserez \\ University Hospital Gasthuisberg, Leuven, Belgium
}


feedback from different national hernia societies was obtained. The Appraisal of Guidelines for REsearch and Evaluation (AGREE) instrument was used by the Cochrane Association to validate the Guidelines. The Guidelines can be used to adjust local protocols, for training purposes and quality control. They will be revised in 2012 in order to keep them updated. In between revisions, it is the intention of the Working Group to provide every year, during the EHS annual congress, a short update of new high-level evidence (randomised controlled trials [RCTs] and metaanalyses). Developing guidelines leads to questions that remain to be answered by specific research. Therefore, we provide recommendations for further research that can be performed to raise the level of evidence concerning certain aspects of inguinal hernia treatment. In addition, a short summary, specifically for the general practitioner, is given. In order to increase the practical use of the Guidelines by consultants and residents, more details on the most important surgical techniques, local infiltration anaesthesia and a patient information sheet is provided. The most important challenge now will be the implementation of the Guidelines in daily surgical practice. This remains an important task for the EHS. The establishment of an EHS school for teaching inguinal hernia repair surgical techniques, including tips and tricks from experts to overcome the learning curve (especially in endoscopic repair), will be the next step. Working together on this project was a great learning experience, and it was worthwhile and fun. Cultural differences between members were easily overcome by educating each other, respecting different views and always coming back to the principles of evidence-based medicine. The members of the Working Group would like to thank the EHS board for their support and especially Ethicon for sponsoring the many meetings that were needed to finalise such an ambitious project.

\section{Guidelines for the treatment of inguinal hernia in adult patients committees}

\section{Steering Committee}

$\begin{array}{ll}\text { Maarten Simons } & \text { Coordinator } \\ \text { Marc Miserez } & \text { EHS contact } \\ \text { Giampiero Campanelli } & \\ \text { Henrik Kehlet } & \end{array}$

Andrew Kingsnorth

Par Nordin

Volker Schumpelick

\section{Working Group}

$\begin{array}{ll}\text { Austria: } & \text { Rene Fortelny } \\ \text { Belgium: } & \text { Marc Miserez } \\ \text { Denmark: } & \text { Morten Bay Nielsen } \\ \text { Finland: } & \text { Timo Heikkinen } \\ \text { France: } & \text { Jean-Luc Bouillot } \\ \text { Germany: } & \text { Joachim Conze } \\ \text { Hungary: } & \text { Georg Weber } \\ \text { Italy: } & \text { Giampiero Campanelli } \\ \text { Netherlands: } & \text { Theo Aufenacker/Maarten Simons } \\ \text { Poland: } & \text { Maciej Smietanski } \\ \text { Spain: } & \text { Salvador Morales-Conde } \\ \text { Sweden: } & \text { Sam Smedberg/Par Nordin } \\ \text { Switzerland: } & \text { Jan Kukleta } \\ \text { United Kingdom: } & \text { Andrew Kingsnorth }\end{array}$

\section{Reference Manager}

Diederik de Lange (NL)

\section{Summary of guidelines on inguinal hernia in adult patients ( $>18$ years)}

Anamnesis Groin swelling, right/left, nature of complaints (pain), duration of complaints, contralateral groin swelling, signs and symptoms of incarceration, reducibility, previous hernia operations.

Predisposing factors: smoking, chronic obstructive pulmonary disease (COPD), abdominal aortic aneurysm, long-term heavy lifting work, positive family history, appendicectomy, prostatectomy, peritoneal dialysis.

Physical examination (Reducible) swelling groin (above the inguinal ligament), differentiation lateral/medial unreliable, operation scar inguinal region, contralateral groin, symptoms of incarceration, reducible, testes, ascites, rectal examination.

Differential diagnosis Swelling: Femoral hernia, incisional hernia, lymph gland enlargement, aneurysm, 
saphena varix, soft-tissue tumour, abscess, genital anomalies (ectopic testis).

Pain: adductor tendinitis, pubic osteitis, hip artrosis, bursitis ileopectinea, irradiating low back pain.

Women: consider femoral hernia, endometriosis.

Diagnostics Clinical investigation. If any (rarely necessary): ultrasound, magnetic resonance imaging (MRI) (with and without Valsalva manoeuvre), herniography.

Treatment Men with asymptomatic or minimally symptomatic inguinal hernia (without or only minimal complaints): consider conservative management.

Incarcerated hernia (no strangulation symptoms): try reduction.

Strangulated hernia: emergency surgery.

Symptomatic inguinal hernia: elective surgery.

Women: consider femoral hernia, consider preperitoneal (endoscopic) approach.

Operation technique (male adults)

Primary Mesh repair: Lichtenstein or unilateral: endoscopic repair are recommended. Endoscopic repair only if expertise is available.

Primary Mesh repair: Committee's

bilateral: recommendation: Lichtenstein or endoscopic.

Recurrent Mesh repair: Committee's

inguinal hernia: recommendation: modify technique in relation to previous technique.

If previously Consider open preperitoneal mesh or anterior: endoscopic approach (if expertise is present).

If previously Consider anterior mesh (Lichtenstein).

- Note 1: The Committee is of the opinion that a totally extraperitoneal (TEP) repair is preferred to a transabdominal preperitoneal (TAPP) approach in the case of endoscopic surgery.

- Note 2: The Committee is of the opinion that, except for the Lichtenstein and endoscopic techniques, none of the alternative mesh techniques have received sufficient scientific evaluation to be given a place in these guidelines.

Prophylactic antibiotics

Anaesthesia

Day surgery
In open surgery, not recommended in low-risk patients. Not recommended in endoscopic surgery.

Most open (anterior) inguinal hernia techniques are eligible for local anaesthesia.

Exclusion considerations: young anxious patients, morbid obesity, incarcerated hernia.

Anterior: all forms of anaesthesia, consider local anaesthesia.

Avoid spinal anaesthesia with high doses of long-acting anaesthetics.

All patients should have long-acting local anaesthetic infiltration preoperatively for postoperative pain control.

ASA I and II: always consider day surgery.

ASA III/IV: consider local anaesthesia, consider day surgery. 
Flow diagram for the treatment of inguinal hernia in

\section{male adults}

Based on a consensus within the Committee.

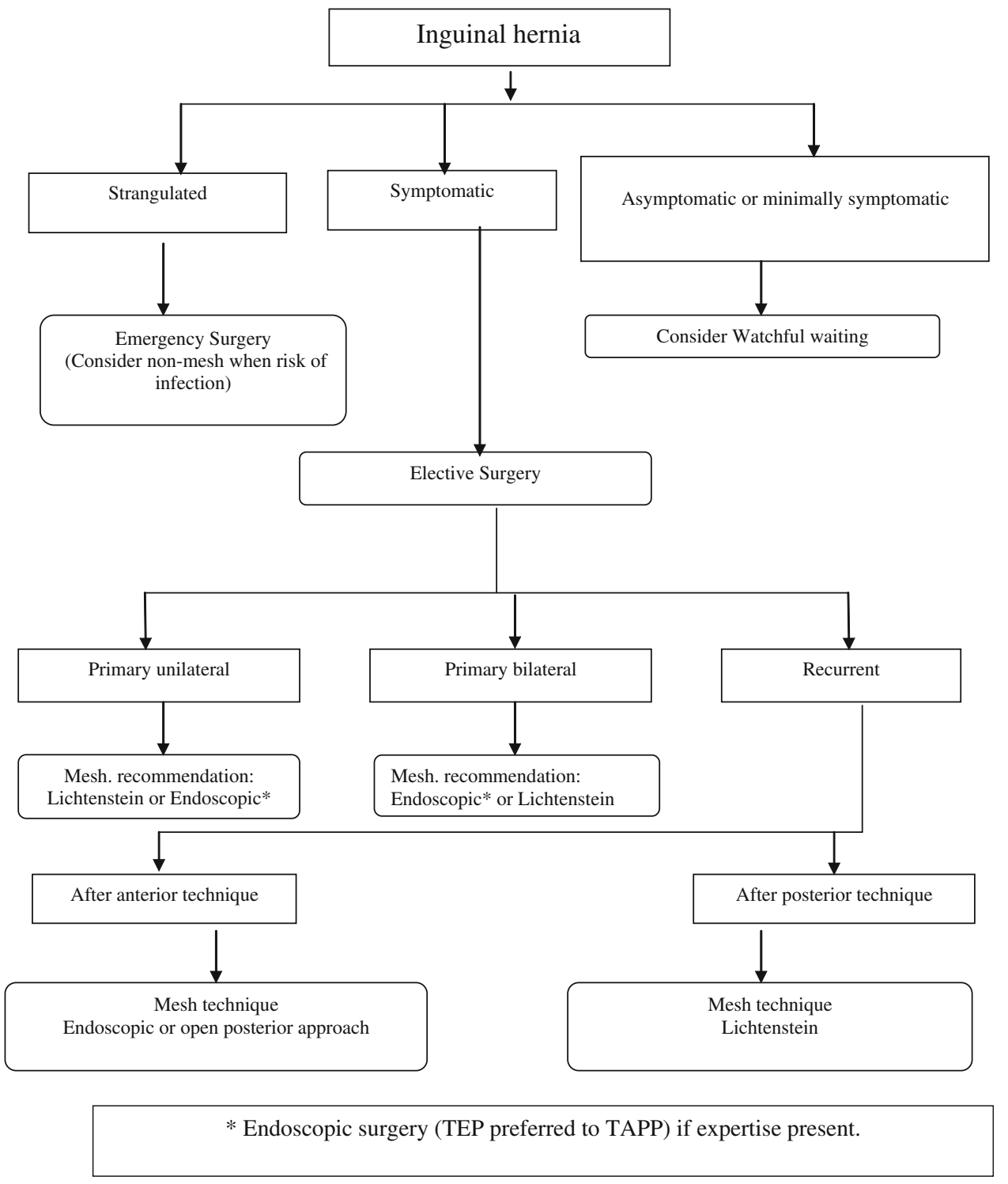

Fig. 1

(Oxford Centre for Evidence-Based Medicine)

Levels of evidence:

1A Systematic review of randomised controlled trials (RCTs) with consistent results from individual (homogenous) studies.

1B RCTs of good quality.

2A Systematic review of cohort or case-control studies with consistent results from individual (homogenous) studies.

2B RCT of poorer quality or cohort or case-control studies.

2C Outcome studies, descriptive studies.

3 Cohort or case-control studies of low quality.

4 Expert opinion, generally accepted treatments.
Grades of recommendation:

A Supported by systematic review and/or at least two RCTs of good quality.

Level of evidence 1A, 1B.

B Supported by good cohort studies and/or case-control studies.

Level of evidence 2A, 2 B.

C Supported by case series, cohort studies of low quality and/or 'outcomes' research.

Level of evidence $2 \mathrm{C}, 3$.

D Expert opinion, consensus committee.

Level of evidence 4. 


\section{All conclusions and recommendations:}

\section{Indications for treatment}

\section{Conclusions}

Level Watchful waiting is an acceptable option for 1B men with minimally symptomatic or asymptomatic inguinal hernias.

Level A strangulated inguinal hernia (with symptoms of 4 strangulation and/or ileus) should be operated on urgently.

\section{Recommendations}

Grade It is recommended in minimally symptomatic or A asymptomatic inguinal hernia in men to consider a watchful waiting strategy.

Grade It is recommended that strangulated hernias are D operated on urgently. It is recommended that symptomatic inguinal hernias are treated surgically.

\section{Non-surgical diagnostics}

\section{Conclusions}

Level In case of an evident hernia, clinical examination 2C suffices.

Differentiation between direct and indirect hernia is not useful. Only cases of obscure pain and/or doubtful swelling in the groin require further diagnostic investigation.

In everyday practice, the sensitivity and specificity of ultrasonography for diagnosing inguinal hernia is low.
A computed tomography (CT) scan has a limited place in the diagnosis of an inguinal hernia.

MRI has a sensitivity and specificity of more than $94 \%$ and is also useful to reveal other musculo-tendineal pathologies.

Herniography has high sensitivity and specificity in unclear diagnosis but has a low incidence of complications. It does not reveal lipomas of the cord.

\section{Recommendations}

Grade It is recommended that groin diagnostic C investigations are performed only in patients with obscure pain and/or swelling.

The flow chart recommended in these cases:

Ultrasound (if expertise is available)

If ultrasound negative $\rightarrow$ MRI (with Valsalva)

If MRI negative $\rightarrow$ consider herniography

\section{Classification}

\section{Recommendations}

Grade It is recommended that the EHS classification for D hernia in the groin is used.

Risk factors and prevention

\section{Conclusions}

Level Smokers, patients with positive family hernia 3 history, patent processus vaginalis, collagen disease, patients with an abdominal aortic aneurysm, after an appendicectomy and prostatectomy, with ascites, on peritoneal dialysis, after long-term heavy work or with COPD have an increased risk of inguinal hernia. This is not proven with respect to (occasional) lifting, constipation and prostatism. 


\section{Recommendations}

Grade Smoking cessation is the only sensible advice $\mathrm{C}$ that can be given with respect to preventing the development of an inguinal hernia.

\section{Treatment of inguinal hernia}

\section{Conclusions}

Level Operation techniques using mesh result in fewer 1A recurrences than techniques which do not use mesh.

The Shouldice hernia repair technique is the best non-mesh repair method.

Endoscopic inguinal hernia techniques result in a lower incidence of wound infection, haematoma formation and an earlier return to normal activities or work than the Lichtenstein technique.

Endoscopic inguinal hernia techniques result in a longer operation time and a higher incidence of seroma than the Lichtenstein technique.

Level Mesh repair appears to reduce the chance of 1B chronic pain rather than increase it. Endoscopic mesh techniques result in a lower chance of chronic pain/numbness than the Lichtenstein technique. In the long term (more than 3 to 4 years follow-up), these differences (nonmesh-endoscopic-Lichtenstein) seem to decrease for the aspect pain but not for numbness.

For recurrent hernias after conventional open repair, endoscopic inguinal hernia techniques result in less postoperative pain and faster reconvalescence than the Lichtenstein technique. Material-reduced meshes have some advantages with respect to long-term discomfort and foreign-body sensation in open hernia repair, but are possibly associated with an increased risk for hernia recurrence (possibly due to inadequate fixation and/or overlap) (Chap. 2.9).
From the perspective of the hospital, an open mesh procedure is the most cost-effective operation in primary unilateral hernias. From a socio-economic perspective, an endoscopic procedure is probably the most cost-effective approach for patients who participate in the labour market, especially for bilateral hernias. In cost-utility analyses including quality of life (QALYs), endoscopic techniques (TEP) may be preferable since they cause less numbness and chronic pain (Chap. 2.18).

Level For endoscopic inguinal hernia techniques, 2A TAPP seems to be associated with higher rates of port-site hernias and visceral injuries, whilst there appear to be more conversions with TEP.

Level There appears to be a higher rate of rare but 2B serious complications with endoscopic repair, especially during the learning curve period.

Other open mesh techniques: Prolene hernia system (PHS), Kugel patch, plug and patch (mesh plug) and Hertra mesh (Trabucco), in short-term follow-up, result in comparable outcome (recurrence) to the Lichtenstein technique. A young man (aged 18-30 years) with a lateral inguinal hernia has a risk of recurrence of at least $5 \%$ following a non-mesh operation and a long follow-up (>5 years) (Chap. 2.8).

Level Endoscopic inguinal hernia techniques with a 2C small mesh $(\leq 8 \times 12 \mathrm{~cm})$ result in a higher incidence of recurrence compared with the Lichtenstein technique.

Women have a higher risk of recurrence (inguinal or femoral) than men following an open inguinal hernia operation due to a higher occurrence of femoral hernias (Chap. 2.7).

The learning curve for performing endoscopic inguinal hernia repair (especially TEP) is longer than that for open Lichtenstein repair, and ranges between 50 and 100 procedures, with the first 3050 being most critical (Chap. 2.12). 
For endoscopic techniques, adequate patient selection and training might minimise the risks for infrequent but serious complications in the learning curve (Chap. 2.12).

There does not seem to be a negative effect on outcome when operated by a resident versus an attending surgeon (Chap. 2.12).

Specialist centres seem to perform better than general surgical units, especially for endoscopic repairs (Chap. 2.12).

Level All techniques (especially endoscopic 4 techniques) have a learning curve that is underestimated.

For large scrotal (irreducible) inguinal hernias, after major lower abdominal surgery, and when no general anaesthesia is possible, the Lichtenstein repair is the preferred surgical technique.

For recurrent hernias, after previous posterior approach, an open anterior approach seems to have clear advantages, since another plane of dissection and mesh implantation is used.

Stoppa repair is still the treatment of choice in case of complex hernias.

\section{Recommendations}

Grade All male adult ( $>30$ years) patients with a A symptomatic inguinal hernia should be operated on using a mesh technique.

When considering a non-mesh repair, the Shouldice technique should be used.

The open Lichtenstein and endoscopic inguinal hernia techniques are recommended as the best evidence-based options for the repair of a primary unilateral hernia, providing the surgeon is sufficiently experienced in the specific procedure.

For the repair of recurrent hernias after conventional open repair, endoscopic inguinal hernia techniques are recommended.

When only considering chronic pain, endoscopic surgery is superior to open mesh.

Grade In inguinal hernia tension-free repair, synthetic A non-absorbable flat meshes (or composite meshes with a non-absorbable component) should be used (Chap. 2.9).
The use of lightweight/material-reduced/largepore $(>1,000-\mu \mathrm{m})$ meshes can be considered in open inguinal hernia repair to decrease longterm discomfort but possibly at the cost of increased recurrence rate (possibly due to inadequate fixation and/or overlap) (Chap. 2.9). It is recommended that an endoscopic technique is considered if a quick postoperative recovery is particularly important (Chap. 2.14).

It is recommended that, from a hospital perspective, an open mesh procedure is used for the treatment of inguinal hernia (Chap. 2.18).

From a socio-economic perspective, an endoscopic procedure is proposed for the active working population, especially for bilateral hernias (Chap. 2.18).

Grade Other open-mesh techniques than Lichtenstein B (PHS, Kugel patch, plug and patch [mesh-plug] and Hertra mesh [Trabucco]) can be considered as an alternative treatment for open inguinal hernia repair, although only short-term results (recurrence) are available.

It is recommended that an extraperitoneal approach (TEP) is used for endoscopic inguinal hernia operations.

It is recommended that a mesh technique is used for inguinal hernia correction in young men (aged 18-30 years and irrespective of the type of inguinal hernia) (Chap. 2.8).

Grade (Endoscopic) hernia training with adequate C mentoring should be started with junior residents (Chap. 2.12).

Grade For large scrotal (irreducible) inguinal hernias, after D major lower abdominal surgery, and when no general anaesthesia is possible, the Lichtenstein repair is the preferred surgical technique. In endoscopic repair, a mesh of at least $10 \times 15 \mathrm{~cm}$ should be considered.

It is recommended that an anterior approach is used in the case of a recurrent inguinal hernia which was treated with a posterior approach. In female patients, the existence of a femoral hernia should be excluded in all cases of a hernia in the groin (Chap. 2.7). 
A preperitoneal (endoscopic) approach should be considered in female hernia repair (Chap. 2.7).

All surgeons graduating as general surgeons should have a profound knowledge of the anterior and posterior preperitoneal anatomy of the inguinal region (Chap. 2.12).

Complex inguinal hernia surgery (multiple recurrences, chronic pain, mesh infection) should be performed by a hernia specialist (Chap. 2.12).

\section{Inguinal hernia in women}

\section{Conclusions}

Level Women have a higher risk of recurrence 2C (inguinal or femoral) than men following an open inguinal hernia operation due to a higher occurrence of femoral hernias.

\section{Recommendations}

Grade In female patients, the existence of a femoral D hernia should be excluded in all cases of a hernia in the groin.

A preperitoneal (endoscopic) approach should be considered in female hernia repair.

Lateral inguinal hernia in young men (aged 18-30 years)

\section{Conclusions}

Level A young man (aged 18-30 years) with a lateral 2B inguinal hernia has a risk of recurrence of at least 5\% following a non-mesh operation and a long follow-up ( $>5$ years).

\section{Recommendations}

Grade It is recommended that a mesh technique is used B for inguinal hernia correction in young men (aged 18-30 years and irrespective of the type of inguinal hernia).

\section{Biomaterials}

\section{Conclusions}

Level Operation techniques using mesh result in 1A fewer recurrences thantechniques which do not use mesh.
Level Material-reduced meshes have some advantages

1B with respect to long-term discomfort and foreign-body sensation in open hernia repair, but are possibly associated with an increased risk for hernia recurrence (possibly due to inadequate fixation and/or overlap).

\section{Recommendations}

Grade In inguinal hernia tension-free repair, synthetic A non-absorbable flat meshes (or composite meshes with a non-absorbable component) should be used. The use of lightweight/material-reduced/largepore $(>1,000-\mu \mathrm{m})$ meshes in open inguinal hernia repair can be considered to decrease long-term discomfort, but possibly at the cost of increased recurrence rate (possibly due to inadequate fixation and/or overlap).

\section{Day surgery}

\section{Conclusions}

Level Inguinal hernia surgery as day surgery is as safe 2B and effective as that in an inpatient setting, and more cost-effective.

Level Inguinal hernia surgery can easily be performed 3 as day surgery, irrespective of the technique used. Selected older and ASA III/IV patients are also eligible for day surgery.

\section{Recommendations}

Grade An operation in day surgery should be considered B for every patient.

\section{Antibiotic prophylaxis}

\section{Conclusions}

Level In conventional hernia repair (non-mesh), 1A antibiotic prophylaxis does not significantly reduce the number of wound infections. NNT 68 .

Level In open mesh repair in low-risk patients, 1B antibiotic prophylaxis does not significantly reduce the number of wound infections. NNT 80 For deep infections, the NNT is 352 . 
Level In endoscopic repair, antibiotic prophylaxis 2B does not significantly reduce the number of wound infections. NNT $\infty$.

\section{Recommendations}

Grade In clinical settings with low rates $(<5 \%)$ of A wound infection, there is no indication for the routine use of antibiotic prophylaxis in elective open groin hernia repair in low-risk patients.

Grade In endoscopic hernia repair, antibiotic B prophylaxis is probably not indicated.

Grade In the presence of risk factors for wound C infection based on patient (recurrence, advanced age, immunosuppressive conditions) or surgical (expected long operating times, use of drains) factors, the use of antibiotic prophylaxis should be considered.

\section{Training}

\section{Conclusions}

Level The learning curve for performing endoscopic 2C inguinal hernia repair (especially TEP) is longer than for open Lichtenstein repair, and ranges between 50 and 100 procedures, with the first 30-50 being the most critical.

For endoscopic techniques, adequate patient selection and training might minimise the risks for infrequent but serious complications in the learning curve.

There does not seem to be a negative effect on outcome when operated by a resident versus an attending surgeon.

Specialist centres seem to perform better than general surgical units, especially for endoscopic repairs.

\section{Recommendations}

Grade (Endoscopic) hernia training with adequate C mentoring should be started with junior residents.
Grade All surgeons graduating as general surgeons D should have a profound knowledge of the anterior and posterior preperitoneal anatomy of the inguinal region.

Complex inguinal hernia surgery (multiple recurrences, chronic pain, mesh infection) should be performed by a hernia specialist.

\section{Anaesthesia}

\section{Conclusions}

Level Open anterior inguinal hernia techniques can 1B be satisfactorily performed under local anaesthetic.

Regional anaesthesia, especially when using high-dose and/or long-acting agents, has no documented benefits in open inguinal hernia repair and increases the risk of urinary retention.

\section{Recommendations}

Grade It is recommended that, in the case of an open A repair, local anaesthetic is considered for all adult patients with a primary reducible unilateral inguinal hernia.

Grade Use of spinal anaesthesia, especially using highB dose and/or long-acting anaesthetic agents, should be avoided.

General anaesthesia with short-acting agents and combined with local infiltration anaesthesia may be a valid alternative to local anaesthesia.

\section{Postoperative recovery}

\section{Conclusions}

Level Endoscopic inguinal hernia techniques result in 1A an earlier return to normal activities or work than the Lichtenstein technique.

\section{Recommendations}

Grade It is recommended that an endoscopic technique A is considered if a quick postoperative recovery is particularly important. 


\section{Aftercare}

\section{Conclusions}

Level The imposition of a temporary ban on lifting, 3 participating in sports or working after inguinal hernia surgery is not necessary. Probably a limitation on heavy weight lifting for 2-3 weeks is enough.

\section{Recommendations}

Grade It is recommended that limitations are not placed $\mathrm{C}$ on patients following an inguinal hernia operation and patients are, therefore, free to resume activities. "Do what you feel you can do." Probably a limitation on heavy weight lifting for $2-3$ weeks is enough.

\section{Postoperative pain control}

\section{Conclusions}

Level Wound infiltration with a local anaesthetic 1B results in less postoperative pain following inguinal hernia surgery.

\section{Recommendations}

Grade Local infiltration of the wound after hernia repair A provides extra pain control and limits the use of analgesics.

\section{Complications}

\section{Recommendations}

Grade It is recommended in the case of open surgery to B operatively evacuate a haematoma which results in tension on the skin.

It is recommended that wound drains are only used where indicated (much blood loss, coagulopathies).

Grade It is recommended that seromas are not C aspirated.

Grade It is recommended that the patient empties his/ D her bladder prior to endoscopic and open operations.
It is recommended that the fascia transversalis/ peritoneum is opened with restrictivity in open surgery of direct hernias. Take care that the bladder might be herniated.

Grade It is recommended that, in the case of large D hernia sacs, transection of the hernia sac is performed and the distal hernia sac is left undisturbed, so as to prevent ischaemic orchitis. Damage to the spermatic cord structures should be avoided.

Grade It is recommended that patients with previous D major lower (open) abdominal intervention or previous radiotherapy of pelvic organs do not undergo endoscopic inguinal hernia surgery.

Grade It is recommended that, due to the risk of D intestinal adhesion and the risk of bowel obstruction, the extraperitoneal approach (TEP) is used for endoscopic inguinal hernia operations. It is recommended that trocar openings of $10 \mathrm{~mm}$ or larger are closed.

Grade It is recommended that the first trocar at D endoscopic hernia surgery (TAPP) is introduced by the open technique.

\section{Chronic pain}

\section{Conclusions; causes and risk factors}

Level The risk of chronic pain after hernia repair with 1B mesh is less than after non-mesh repair. The risk of chronic pain after endoscopic hernia repair is lower than after open hernia repair.

Level The overall incidence of moderate to severe chronic

$2 \mathrm{~A}$ pain after hernia surgery is around $10-12 \%$. The risk of chronic pain after hernia surgery decreases with age.

Level Preoperative pain may increase the risk of 2B developing chronic pain after hernia surgery. Preoperative chronic pain conditions correlate with the development of chronic pain after hernia surgery. 
Severe early postoperative pain after hernia surgery is correlated to the development of chronic pain.

Females have an increased risk of developing chronic pain after hernia surgery.

\section{Conclusions; prevention of chronic pain}

Level Material-reduced meshes have some advantages 1B with respect to long-term discomfort and foreign-body sensation in open hernia repair (when only considering chronic pain).

Level Prophylactic resection of the ilioinguinal nerve 2A does not reduce the risk of chronic pain after hernia surgery.

Level Identification of all inguinal nerves during open 2B hernia surgery may reduce the risk of nerve damage and postoperative chronic groin pain.

\section{Conclusions; treatment of chronic pain}

Level A multidisciplinary approach at a pain clinic is an 3 option for the treatment of chronic postherniorrhaphy pain.

Surgical treatment of specific causes of chronic post-herniorrhaphy pain can be beneficial for the patient, such as the resection of entrapped nerves, mesh removal in mesh-related pain, removal of endoscopic staples or fixating sutures.

\section{Recommendations}

Grade The use of lightweight/material-reduced/largeA pore $(>1,000-\mu \mathrm{m})$ meshes in open inguinal hernia repair can be considered to decrease long-term discomfort (when only considering chronic pain).

Endoscopic surgery is superior to open mesh (when only considering chronic pain), if a dedicated team is available.

Grade It is recommended that risks of development of B chronic postoperative pain are taken into account when the method of hernia repair is decided upon.
It is recommended that inguinal nerves at risk (three nerves) are identified at open hernia surgery.

Grade It is recommended that a multidisciplinary C approach is considered for the treatment of chronic pain after hernia repair.

It is recommended that the surgical treatment of chronic post-herniorrhaphy pain as a routine is restricted in the lack of scientific studies evaluating the outcome of different treatment modalities.

\section{Mortality}

\section{Recommendations}

Grade It is recommended to offer patients with femoral B hernia early planned surgery, even if the symptoms are vague or absent.

Grade It is recommended to intensify efforts to improve D the early diagnosis and treatment of patients with incarcerated and or strangulated hernia.

\section{Costs}

\section{Conclusions}

Level From the perspective of the hospital, an open 1B mesh procedure is the most cost-effective operation in primary unilateral hernias. From a socio-economic perspective, an endoscopic procedure is probably the most cost-effective approach for patients who participate in the labour market, especially for bilateral hernias. In cost-utility analyses including quality of life (QALYs), endoscopic techniques (TEP) may be preferable, since they cause less numbness and chronic pain.

\section{Recommendations}

Grade It is recommended that, from a hospital A perspective, an open mesh procedure is used for the treatment of inguinal hernia.

From a socio-economic perspective, an endoscopic procedure is proposed for the active working population, especially for bilateral hernias. 


\section{General}

\section{Introduction}

One of the aims of the European Hernia Society (EHS) is the development and implementation of specialised medical guidelines for hernia management. Guidelines are not only important for clinical practice, but also for (postgraduate) training, the registration of complications and the development of indicators. The process of developing guidelines can also direct scientific research, as it indicates the areas in which there is a lack of evidence for clinical practice.

Guidelines are:

An agreed line of conduct for appropriate care within the professional group, which is based as much as possible on scientific insights from systematic and current clinical research into the efficacy and effectiveness of the available alternatives, taking the patient situation into account.

Guidelines are developed to:

- Improve medical quality and effectiveness (management)

- Reduce the variation between physicians: the practice must be based more on evidence than on experiences or opinions (professionalism versus intuition)

- Make practice more transparent (accountability: who can expect what from whom?)

Improving the results of inguinal hernia treatment will have major medical and economic consequences. For the patient, a successful inguinal hernia repair means a lower risk of complications, a quick postoperative recovery and a minimal risk of persistent pain symptoms or recurrent hernias. Of course, the individual patients' situation and the general costs of the treatment continue to be major considerations.

These guidelines concerning the treatment of inguinal hernia have been developed and are owned by the European Hernia Society (EHS). Development of the Guidelines was financed through a grant by Ethicon.

\section{Motivation}

In 2003, the Dutch Society of Surgeons published evidence-based guidelines for the treatment of inguinal hernias. The Dutch Society of Hernia proposed in 2005 to have the Guidelines translated and have some international experts in the field judge whether the Guidelines could be suitable for use by the EHS. A Steering Committee was installed and, after reading and commenting on the contents, it was agreed that they would be used as the base for the EHS Guidelines. A Working Group was formed.
Ethicon agreed to sponsor the development. Ethicon would not become the owner and would not interfere in the methods and contents, thus, avoiding bias.

All member countries of the EHS were asked to name a representative to join the Working Group.

\section{Objective}

These guidelines form a document with recommendations to support the daily practice of the treatment of inguinal hernias by surgeons. These guidelines are based on the results of scientific research and the formation of opinions arising from this which are aimed at emphasising good clinical practice. These guidelines are intended as a reference manual for daily practice. These guidelines provide starting points for the drawing up of local protocols, which promote their implementation and serve as a base or tool for education and training in groin hernia surgery. The potential health benefit is an improvement of the level of care for patients with inguinal hernia by reducing complications like recurrence and chronic pain.

\section{Definition}

An inguinal hernia or hernia inguinalis is a protrusion of the contents of the abdominal cavity or preperitoneal fat through a hernia defect in the inguinal area, irrespective of whether this is preformed (congenital). This situation can give rise to complaints such as discomfort and pain. Sometimes, it is not possible to reduce the contents of the hernia sac (non-reducible hernia). In the case of a narrow hernia defect, there is a risk of the hernia sac contents becoming incarcerated, resulting in an obstruction of the intestine (ileus) and/or a circulatory disorder of the incarcerated content (strangulation), which can lead to necrosis and possible perforation of the intestine.

A recurrent inguinal hernia is a swelling due to a defect in the inguinal region where an inguinal hernia operation was previously performed.

\section{Target population}

The target population was all adult ( $>18$ years of age) patients with a primary or recurrent inguinal hernia (asymptomatic or symptomatic, acute or elective). The Guidelines concern male patients unless stated otherwise.

Description of problem and initial questions

The committee which prepared these guidelines wished to gain answers to the following (deemed as the most important) questions that are known to give rise to discussion: 
a. What are the indications for inguinal hernia treatment? Is operative treatment necessary?

b. What is the best technique for the treatment of an inguinal hernia (considering factors such as recurrence, complications, postoperative recovery, pain, costs)? What mesh is best?

c. What are the complications of the various techniques, and how can these be treated? What causes pain complications and how to treat these?

d. What is the best form of anaesthetic? Should local anaesthesia be recommended as the first choice?

e. Can an inguinal hernia be operated in ambulatory surgery? Thus, decreasing cost, possibly improving quality?

f. Is the routine use of antibiotics necessary?

Inguinal hernia treatment for adults in Europe in 2007

A number of studies provide insight into the treatment techniques which surgeons have used since 1992. Endoscopic surgery entered the scene in 1991 and the Lichtenstein technique around 1993. After 1993, other mesh techniques followed, such as plug and patch, PHS etc.

In many European countries, studies were performed to evaluate the different techniques used [32, 121, 134].

Table 1

\begin{tabular}{|c|c|c|c|c|c|}
\hline \multirow[t]{2}{*}{ Country } & \multirow[t]{2}{*}{ Year } & \multicolumn{4}{|c|}{ Types of inguinal hernia repair } \\
\hline & & $\begin{array}{l}\text { Conventional } \\
(\%)\end{array}$ & $\begin{array}{l}\text { Open } \\
\text { mesh }(\%)\end{array}$ & $\begin{array}{l}\text { Endoscopic } \\
(\%)\end{array}$ & $\begin{array}{l}\text { Other } \\
(\%)\end{array}$ \\
\hline Netherlands & 2006 & 4 & 77 & 19 & \\
\hline Denmark & 2006 & 2.5 & 82.5 & 15 & \\
\hline Finland & 2006 & 7 & 81 & 8 & 3 \\
\hline France & 2006 & 14.9 & 46 & 34 & 4.6 \\
\hline Poland & 2006 & 38 & 60 & 1 & \\
\hline Austria & 2006 & 76 & & 24 & \\
\hline Hungary & 2007 & 60 & 34 & 6 & \\
\hline Sweden & 2006 & 8.5 & 82 & 9 & \\
\hline
\end{tabular}

Provided by the Working Group

Many different techniques and strategies are used, reflecting different cultures, insights and economics.

Transparency of the process and method

The Steering Committee first met in Torino in December 2005. A Working Group was installed. The Working Group participated in a two-day workshop in Amsterdam in April
2007. A short course in evidence-based guideline development (EBGD) and clinical appraisal was followed, after which all relevant literature was searched in the Cochrane Database, Medline and Embase.

In September 2007, the Working Group had a one-day meeting in Amsterdam. Participation in the course "Evidence-Based Guideline Development" by Dr. Anco Vahl was desirable.

All chapters were divided among participants and two were assigned to each. According to evidence-based medicine guidelines, the quality was assessed.

The concept chapters were discussed and, where necessary, consensus was found, after which recommendations were agreed upon. From December 2007 till March 2008, comments from all participants were gathered via email by Maarten Simons and Prof. Marc Miserez. Prof. Andrew Kingsnorth edited and commented on the concept guidelines in March 2008. The March concept was sent to all participating countries for the national commentary phase. A third meeting was organised at the EHS meeting in Sevilla, May 2008. During a session, all chapters were presented by the respective authors. In the summer of 2008, minor comments were used to finalise the Guidelines. The Steering Committee agreed to the Guidelines, after which the results were published in Hernia and on the Internet (http://www.herniaweb.org).

Working Group members

When the Working Group was appointed, members with the following characteristics were sought:

- Clinical and scientific expertise in the area of inguinal hernia surgery

- Members drawn from as many European countries as possible

- Members drawn from university and non-university hospitals and teaching and non-teaching hospitals

- Supporters of as many different operation techniques as possible

- Epidemiological expertise

- No conflicts of interest concerning the contents of the guidelines

Members of the Steering Committee and Working Group.

\section{Steering Committee}

Dr. Maarten Simons, MD, PhD, general surgeon, Onze Lieve Vrouwe Gasthuis (OLVG) Hospital, Amsterdam; District training hospital, thesis "Shouldice in Amsterdam," Chairman of the Dutch Guidelines Committee on 
Inguinal Hernia Treatment, 30 publications. Expert in Lichtenstein and TEP.

Prof. Marc Miserez, general surgeon, Associate Professor of surgery, University Hospital Gasthuisberg, Leuven Belgium; secretary scientific research EHS board, 10 publications. Expert in Lichtenstein and endoscopic (TEP).

Prof. Giampiero Campanelli, Full Professor of Surgery, University of Insubria-Varese Chief Department of General Surgery II Day and Week-Surgery Multimedica Santa Maria Hospital in Castellanza General Secretary of European Hernia Society.

General Secretary of the Italian Society of Ambulatory Surgery and Day-Surgery.

President of Fondazione Day-Surgery Onlus, 100 hernia publications, two books on hernia.

Prof. Andrew Kingsnorth, general, gastrointestinal (GI) and abdominal wall surgeon, Derriford Hospital, Plymouth. University Hospital. President of the EHS. More than 60 publications and a hernia textbook. Open hernia surgeon. Special interest in RCTs.

Dr. Pär Nordin, MD, PhD, general surgeon, Östersund Hospital, Östersund, Sweden. Head of the Swedish Hernia Register. Thesis on "Anaesthesia and surgical techniques in groin hernia surgery." Nineteen publications. Special interest in register-based studies in groin hernia surgery.

Prof. Volker Schumpelick, general surgeon, head of Aachen University surgical department, more than 500 publications, multiple books, editor in chief of Hernia.

\section{Working Group}

Dr. Theo Aufenacker, general surgeon, Rijnstate Hospital Arnhem, thesis "The Lichtenstein Inguinal Hernia Repair," 10 publications.

Prof. Jean Luc Bouillot, Professor of general surgery, University Descartes, Paris. President of the French chapter of the EHS, expert in abdominal wall surgery (conventional and endoscopic). More than 50 contributions at conferences.

Dr. Joachim Conze, general surgeon, Aachen University, publications, expert in open and endoscopic surgery. Thirty-two publications, several chapters in different hernia textbooks, general secretary of the German Hernia Society, special interest in open incisional hernia, biomaterials and RCTs.

Dr. Rene Fortelny, general and visceral surgeon, Chief of the Hernia Center at Wilhelminenspital, Vienna; board member of the Austrian Hernia Society and $\mathrm{Zu}$ erser Hernienforum, team leader of the Experimental Hernia Group at the Ludwig Boltzmann Institute for Experimental and Clinical Traumatology, Austrian
Center of Tissue Regeneration/Vienna. Fifteen publications. Expert in TAPP and Lichtenstein.

Dr. Timo Heikkinen, associate Professor of Oulu University Hospital. Fourteen hernia publications. Expert in Lichtenstein, TEP and TAPP.

Dr. Jan Kukleta, general, visceral, abdominal wall surgeon, Klinik Im Park, Zurich, Switzerland. Member of the European-, American-, AsiaPacific Hernia Society. President of the Swiss Association for Hernia Surgery, Lecturer at the European Surgical Institute Hamburg and Elancourt Paris. Specialist in advanced endoscopic procedures, expert in endoscopic groin and abdominal wall repair. Director of the Endoscopic Training Center in Zurich. More than 50 hernia-specific contributions at international congresses on four continents.

Dr. Morten Bay Nielsen, General Surgeon. Hvidovre University Hospital Copenhagen, 36 hernia publications, secretary of the Danish Database.

Dr. Salvador Morales-Conde, MD, PhD, Associate Professor of Surgery of the University of Sevilla. Chief of the Advanced Endoscopic Unit of the University Hospital Virgen del Rocío. President of the Spanish Chapter of Abdominal Wall Surgery of the Spanish Association of Surgery. General secretary of the Spanish Chapter of Endoscopic Surgery of the Spanish Association of Surgery.

Dr. Sam Smedberg, MD, PhD, general surgeon, Helsingborg Hospital, Helsingborg, Sweden. County Hospital. Thesis 1986 on "Herniography and Hernia Surgery." Thirty-five publications. Expert in Lichtenstein, open preperitoneal repair, Shouldice. Special interest in groin pain problems.

Dr. Maciej Smietanski, MD, PhD, general surgeon, at the Department of General, Endocrine Surgery and Transplantation of the Medical University of Gdansk, Poland. Leader of the Polis Hernia Study Group. Thesis "Lichtenstein versus mesh-plug inguinal hernia repairRCT of 1 year follow-up." Seventeen publications on hernia surgery and author of the Polish Standard for Groin Hernia Repair.

Dr. György Weber, MD, PhD, Professor of Surgery, Department of Surgery, Director, Department of Surgical Research and Techniques, Medical Faculty University of Pécs general and vascular surgeon, expert in TAPP, Lichtenstein and endoscopic incisional hernia, 22 publications in hernia surgery.

\section{Reference Manager}

Dr. Diederik de Lange, resident of general surgery, researcher of guidelines on inguinal hernia, four publications on inguinal hernia. 
Owner and legal significance

\section{Owner}

These guidelines are the property of the European Hernia Society.

\section{Legal significance}

Guidelines are not legal requirements, but are evidence-based insights and recommendations in order to provide qualitatively good care. In this, it is important to realise that there are different "levels of evidence," varying from the highest level (1A), which has been consistently demonstrated by systematic review, and the lowest level (4), which is only based on the opinion of experts. This results in different classes of recommendation. As these recommendations are based on the "average patient," care providers can, where necessary, deviate from the guidelines in accordance with their professional opinion. Indeed, this can sometimes be necessary if the patient's situation requires that.

When the guidelines are not followed, this should be justified and documented.

Intended (target) users

These guidelines are primarily intended for surgeons and trainee surgeons.

Some chapters are also intended for other providers, such as general practitioners, who wish to provide information to patients with an inguinal hernia.

\section{Collection and assessment of the literature}

All relevant literature until April 2007 (Medline, Embase and Cochrane) was prepared by small groups and assessed by all Working Group members. The literature of all level 1A and/or 1B studies was searched during the development of the Guidelines until May 2008. The Oxford Centre for Evidence-Based Medicine was used. After this, a consensus, where necessary, was reached and the conclusions and recommendations were formulated. For all articles, in accordance with evidence-based guidelines criteria, two surgeons always determined whether or not an article was relevant (according to possible bias). Each time, a unanimous final opinion was sought and this was always realised. The Working Group met on three occasions. For chapters in which only level $2 \mathrm{C}$ or 3 articles were available, it was difficult to choose the best evidence from, at times, hundreds of articles. Search bias in these cases cannot be excluded.

Levels of evidence:

1A Systematic review of RCTs with consistent results from individual (homogenous) studies.

1B RCTs of good quality.

2A Systematic review of cohort or case-control studies with consistent results from individual (homogenous) studies.

2B RCT of poorer quality or cohort or case-control studies.

2C Outcome studies, descriptive studies.

3 Cohort or case-control studies of low quality.

4 Expert opinion, generally accepted treatments.

Grades of recommendation:

A Supported by systematic review and/or at least 2 RCTs of good quality.

Level of evidence 1A, 1B.

B Supported by good cohort studies and or case-control studies.

Level of evidence 2A, 2B.

C Supported by case series, cohort studies of low quality and/or 'outcomes' research.

Level of evidence $2 \mathrm{C}, 3$.

D Expert opinion, consensus committee.

Level of evidence 4.

Description of the implementation trajectory

For the Dutch Guidelines that were published in 2003, an implementation study and a pilot study among targetted users were performed.

A national inventory of all inguinal hernia operations carried out in two periods was performed. The first period was a "baseline measurement" in the period prior to the publication of the Guidelines (January to March of 2001) and the second period was quite some time after the publication of the Guidelines (January to March of 2005). By means of the registration forms, the number of inguinal hernia operations carried out in all of the hospitals in these periods were counted (see registration form). The same system will be implemented on a European basis. A prospective database will be necessary for this. Plans for such a registration system are under development. In the Guidelines, operative methods and a registration form are proposed (Appendix 2). The EHS is developing a skills and 
teaching institute to facilitate and train surgeons and residents to be able to work according to the guidelines.

Procedure for authorising guidelines with the European Hernia Society

Guidelines should be developed on the basis of results from scientific research and opinions related to this which are aimed at making good medical practice more explicit. In addition to this, there should be a broad level of support within the EHS.

Applicability and costs

A pilot study among targetted users was performed in two large district hospitals in the Netherlands in 2002 [24]. There were no barriers to implementation either in costs or logistical possibilities. There are, possibly, European countries where certain hospitals cannot afford endoscopic hernia surgery.
Expiry date

The Guidelines are valid until 1st January 2012. Updating of the Guidelines (RCT literature) will be performed continuously by the two authors of each chapter, with a yearly meeting at the EHS at which the publication of relevant updates will be decided upon.

\section{Validation}

The Appraisal of Guidelines for REsearch and Evaluation (AGREE) instrument was used to validate the Guidelines.

Almost all criteria were fulfilled. Review was performed by four external experts in surgery and epidemiology. Two members of the Dutch Cochrane Institute performed a rigorous analysis, which led to many adjustments (Appendix 6). 


\section{Guidelines for the treatment of inguinal hernia in adults}

The groin is a naturally weak point in the abdominal wall. This weakness in the inguinal region is referred to anatomically as the myopectineal orifice of Fruchaud. Cranially and medially, this is bordered by the conjoined tendon and the rectus abdominis muscle, laterally by the iliopsoas muscle and caudally by the superior ramus of the os pubis [104]. This area is covered by the fascia transversalis, split into two by the inguinal ligament and penetrated by the spermatic cord (in men)/round ligament (in women) and femoral vessels. The integrity of the area is, therefore, determined solely by the fascia transversalis. Penetration of a peritoneal hernia sac (or preperitoneal lipoma) through the orifice is referred to as a hernia. The failure of the fascia transversalis to retain the peritoneum/preperitoneal fat is, therefore, the fundamental cause of an inguinal hernia. This fascia is weakened by congenital or acquired factors on the one hand and pressure increasing events on the other.

Inguinal hernias are corrected by repairing the fascial defect in the myopectineal orifice of Fruchaud or by reinforcing the weakened fascia transversalis and bridging the defect by inserting a prosthesis (mesh).

\section{Indications for treatment}

Authors: Jean Luc Bouillot and Maarten Simons

What are the indications for a surgical treatment of inguinal hernia?

Can a non-surgical (conservative) treatment be considered?

Search terms: inguinal hernia, treatment.

\section{Conclusions}

Level Watchful waiting is an acceptable option for 1B men with minimally symptomatic or asymptomatic inguinal hernias.

Level A strangulated inguinal hernia (with symptoms of 4 strangulation and/or ileus) should be operated on urgently.

\section{Recommendations}

Grade It is recommended in minimally symptomatic or A asymptomatic inguinal hernia in men to consider a watchful waiting strategy.

Grade It is recommended that strangulated hernias are D operated on urgently.

It is recommended that symptomatic inguinal hernias are treated surgically.
The incidence and prevalence of inguinal hernia are not precisely known [263]. The chance of a person having to undergo an inguinal hernia operation during his/her life is quite high, $27 \%$ in the case of men and $3 \%$ in the case of women [248]. As almost all diagnosed inguinal hernias are operated on, the natural course of an untreated inguinal hernia is scarcely known. Spontaneous recovery has never been described in adults.

An inguinal hernia is operated in order to reduce the symptoms, when acute complications occur or to prevent complications.

\section{Table 1 Definitions}

\begin{tabular}{|c|c|}
\hline $\begin{array}{l}\text { Asymptomatic } \\
\text { inguinal } \\
\text { hernia }\end{array}$ & $\begin{array}{l}\text { Inguinal hernia without pain } \\
\text { or discomfort for the patient }\end{array}$ \\
\hline $\begin{array}{l}\text { Minimally } \\
\text { symptomatic } \\
\text { hernia }\end{array}$ & $\begin{array}{l}\text { Inguinal hernia with complaints that do not } \\
\text { interfere with daily normal activities }\end{array}$ \\
\hline $\begin{array}{l}\text { Symptomatic } \\
\text { inguinal } \\
\text { hernia }\end{array}$ & Inguinal hernia which causes symptoms \\
\hline $\begin{array}{l}\text { Non-reducible } \\
\quad \text { inguinal } \\
\text { hernia }\end{array}$ & $\begin{array}{l}\text { Inguinal hernia in which the contents of } \\
\text { the } \\
\text { sac cannot be reduced into the } \\
\text { abdominal } \\
\text { cavity; this can be in chronic cases } \\
\text { (accreta) or acute cases (incarceration) }\end{array}$ \\
\hline $\begin{array}{l}\text { Strangulated } \\
\text { inguinal } \\
\text { hernia }\end{array}$ & $\begin{array}{l}\text { Inguinal hernia which is non-reducible } \\
\text { (incarcerated) and shows symptoms of } \\
\text { strangulation (vascular disorders of the } \\
\text { hernia content) and/or ileus }\end{array}$ \\
\hline
\end{tabular}

\section{Asymptomatic inguinal hernia}

An asymptomatic inguinal hernia is operated on to prevent strangulation. An emergency operation due to a strangulated inguinal hernia has a higher associated mortality than an elective operation ( $>5$ vs. $<0.5 \%$ ) [32, $219]$, yet, it is not clear whether the elective operation of all inguinal hernias would have a significant impact on the life expectancy of patients with an inguinal hernia [243].

The literature reveals that the majority of patients with strangulation either did not know they had an inguinal hernia or had not sought medical attention for their condition [107, 200, 250, 251]. Furthermore, the chance of incarceration is sufficiently low (estimated to be $0.3-3 \%$ per year) that the policy of operating on every inguinal hernia, particularly in the case of elderly patients, could, in fact, lead to a higher morbidity and mortality [107, 243]. 
Incarceration occurs at least ten times more often in the case of indirect hernias than direct hernias. However, it is difficult to clinically distinguish a indirect hernia from a direct hernia [148, 202, 252, 278].

Two level 1B RCTs have been published, comparing operation versus watchful waiting.

In the Fitzgibbons trial, in which 356 men (over 18 years of age) were assigned to operation and 366 men were assigned to watchful waiting (WW), the main conclusions after 2 years of follow-up were: $23 \%$ crossover from WW to operation, one acute incarceration without strangulation within 2 years and one incarceration with bowel obstruction within 4 years [100]. There were no differences in pain.

In the O'Dwyer trial, in which 80 men (over 55 years of age) were randomised to operation and 80 to $\mathrm{WW}$, the main conclusions after 1 year of follow-up were: 23/80 (29\%) patients crossed over from observation to operation and three serious hernia-related adverse events occurred in the WW group [226]. One crossover patient had a postoperative myocardial infarction and died, one patient had a postoperative stroke and one patient had an acute hernia. Both patients that had a serious postoperative event had comorbid cardiovascular disease which had deteriorated significantly in the period under observation. Had they been operated on at presentation, such an event may have been avoided.

The results of both trials are not conclusive and differ slightly; however, watchful waiting is an acceptable option for men with asymptomatic or minimally symptomatic inguinal hernias. Incarcerations occur rarely. In one trial, it was concluded that (elderly) men with significant comorbidity could benefit from an operation electively in order to reduce the risks of increase in this morbidity and a higher (operative) mortality when operated in an emergency setting.

\section{Symptomatic/non-reducible inguinal hernia}

Symptomatic inguinal hernias give rise to symptoms of discomfort and/or pain. Large hernias can give rise to cosmetic complaints. Symptomatic inguinal hernias are operated on electively to reduce complaints and/or to prevent complications. Non-reducible hernias without complaints of incarceration have a theoretically higher chance of strangulation.

\section{Strangulated inguinal hernia}

Depending on the definition used, the rate of incarceration/ strangulation is estimated to be $0.3-3 \%$ per year $[107,122$, $214,251]$. There is possibly some increased risk accumulation during the first year after the hernia development
[107, 251]. It is not possible to adequately assess the vitality of the strangulated hernia content by means of physical examination. Strangulated hernia is an indication for emergency surgical treatment.

\section{Non-surgical diagnostics}

Authors: Giampiero Campanelli and György Weber

Which diagnostic modality is the most suitable for diagnosing inguinal hernia in patients with groin complaints (without clear swelling in the groin region)?

Search terms: inguinal hernia, diagnosis, herniography, MRI, ultrasound, CT scan, laparoscopy, combinations.

\section{Conclusions}

Level In case of an evident hernia, clinical examination $2 \mathrm{C}$

Differentiation between direct and indirect hernia is not useful; only cases of obscure pain and/or doubtful swelling in the groin require further diagnostic investigation.

In everyday practice, the sensitivity and specificity of ultrasonography for diagnosing inguinal hernia is low.

A CT scan has a limited place in the diagnosis of an inguinal hernia.

MRI has a sensitivity and specificity of more than $94 \%$ and is also useful to reveal other musculo-tendineal pathologies.

Herniography has high sensitivity and specificity in unclear diagnosis, but has a low incidence of complications. It does not reveal lipomas of the cord.

\section{Recommendations}

Grade It is recommended that groin diagnostic C investigations are performed only in patients with obscure pain and/or swelling.

The flow chart recommended in these cases:

Ultrasound (if expertise is available)

If ultrasound negative $\rightarrow$ MRI (with Valsalva)

If MRI negative $\rightarrow$ consider herniography

\section{Diagnosis}

The diagnosis of inguinal hernia can be established by means of physical examination with a sensitivity of 74.5$92 \%$ and a specificity of $93 \%$ [166, 306].

Doubts about the diagnosis can exist in the case of a vague groin swelling, vague localisation of the swelling, 
intermittent swelling which is not palpable during examination and obscure groin complaints without swelling.

A hernia with clear clinical features does not require any further investigation.

Differentiating the type of hernia (direct-indirect-femoral) using well-described anatomical landmarks is necessary only to diagnose femoral hernia, as this is important to prioritise an operation. Differentiating medial from lateral hernia is unreliable [148, 202, 252, 278]. Almost all of these patients will proceed to surgical exploration and repair. There are almost no studies with a good diagnostic gold standard because only patients with a positive finding undergo surgery.

\section{Ultrasonography}

Ultrasonography is a useful non-invasive adjunct to physical examination. In clinical occult groin hernia, ultrasound specificity in relation to surgical exploration is $81-100 \%$, its sensitivity is $33 \%$ and up to $100 \%$ in clinical diagnosis of a groin hernia [10, 45, 189, 260, 299, 306].

\section{CT scan}

CT scan does not have a significant role in the diagnosis of inguinal hernia, even though it has a sensitivity of $83 \%$ and a specificity of $67-83 \%$ [136].

It is useful in the rare case of involvement of the urinary bladder [9, 18, 63, 307].

\section{MRI}

The advantage of MRI is that other pathologies can also be diagnosed (inflammation, tumour) [179].

MRI can show an accurate and early diagnosis of the different sport-related pathologies [28].

MRI imaging can be used to perform imaging in any plane and dynamic examinations during straining. Its sensitivity is $94.5 \%$ and specificity is $96.3 \%$ [306].

\section{Herniography}

Herniography is safe, sensitive (100\%) and specific (98$100 \%)$ in occult hernia [55, 108, 119, 123, 133, 191].

Herniography does not identify a potential lipoma of the cord which can cause groin pain and/or obscure swelling.

In many articles, a good reference standard (operation) is lacking. For $12-54 \%$ of the herniographies which are carried out in patients without swelling, a hernia is diagnosed [127].

An occult hernia can be found with herniography in $25 \%$ athletes with long-standing undefined groin pain [152].
The risk of complications is $0-4.3 \%$, and these include contrast allergy, puncture of the intestine, abdominal wall haematoma and short-lasting pain [127, 146, 212]. In cases of obscure pain in the groin with an uncertain diagnosis of inguinal hernia, an initial time of 4 months (in the absence of clinical deterioration) is worthwhile before proceeding to herniography [55].

\section{Differential diagnosis}

The differential diagnosis of the swelling in the groin:

- Inguinal (recurrent) hernia

- Femoral hernia

- Incisional hernia

- Lymph gland enlargement

- Aneurysm

- Varix (vena saphena magna)

- Soft-tissue tumour

- Abscess

- Genital anomalies (ectopic testis)

- Endometriosis

The differential diagnosis in pain without typical swelling:

- Adductor tendinitis

- Pubic osteitis

- Hip artrosis

- Bursitis Ileopectinea

- Irradiating low back pain

- Endometriosis

\section{Classification}

Author: Giampiero Campanelli

Is it necessary to classify inguinal hernias and which classification is the most suitable?

Search terms: inguinal hernia, classification.

\section{Recommendations}

Grade It is recommended that the EHS classification for D hernia in the groin is used.

An unequivocal classification of inguinal hernias is important for the rational choice of treatments (in case of tailored surgery) and for the analysis of scientific data. It is also possible to compare the various treatments in clinical trials when hernias can be classified in an unequivocal manner.

Current inguinal hernia classifications are numerous: traditional (medial/lateral/recurrent), Nyhus, Gilbert, Rutkow/Robbins, Schumpelick, Harkins, Casten, Halverson 
and McVay, Lichtenstein, Bendavid, Stoppa, Alexandre, Zollinger Unified [60, 224, 324].

As it is important that a classification system is simple to use and remember, the guidelines committee advises the EHS classification [208]. This classification can be found on the website of the EHS

\section{European Hernia Society Classification}

Table 2

\begin{tabular}{llllll}
\hline $\begin{array}{l}\text { EHS Groin Hernia } \\
\text { Classification }\end{array}$ & \multicolumn{5}{c}{ Primary/recurrent } \\
\hline & 0 & 1 & 2 & 3 & $\mathrm{X}$ \\
Lateral (L) & & & & & \\
Medial (M) & & & & & \\
Femoral (F) & & & & & \\
\hline
\end{tabular}

One classification system for recurrent hernia has been described by Campanelli et al. [60].

\section{Risk factors and prevention}

Authors: Maciej Smietanski and Jean Luc Bouillot

What are the risk factors for developing an inguinal hernia and are there preventive measures?

Search terms: inguinal hernia, risk factors.

\section{Conclusions}

Level Smokers, patients with positive family hernia 3 history, patent processus vaginalis, collagen disease, patients with an abdominal aortic aneurysm, after an appendicectomy and prostatectomy, with ascites, on peritoneal dialysis, after long-term heavy work or with COPD have an increased risk of inguinal hernia. This is not proven with respect to (occasional) lifting, constipation and prostatism.

\section{Recommendations}

Grade Smoking cessation is the only sensible advice C that can be given with respect to preventing the development of an inguinal hernia.

Textbooks mention many risk factors for the development of an inguinal hernia or a recurrence. Smoking is almost certainly a risk factor [239, 283]. People with abnormal collagen metabolism (also known among smokers) have an increased risk, which is also revealed in a higher incidence of inguinal hernias in patients with aortic aneurysm. Also, patent processus vaginalis is a risk factor [181, 239, 308]. Abnormal collagen metabolism possibly clarifies the fact that there are families with an abnormally large number of hernias of all types [157]. In one casecontrol study, the family history of hernia seems to be the only independent risk factor of hernia [177]. Chronic coughing (COPD) seems to be a risk factor [62].

Additional risk has not been demonstrated for prostatism and constipation. Although the majority of studies reveal that physical work is not a risk factor, two retrospective case-control studies revealed that long-term and heavy work does increase the risk of hernias $[62,101]$. A case-control study among women did not demonstrate this, and this was also the case for smoking, whereas many sports and obesity were protecting factors. In this study, constipation and a positive family history were positive risk factors [188]. A low (cosmetic) incision for appendectomy can disrupt the shutter mechanism and increase the risk of an inguinal hernia on the right-hand side [297].

Ascites and peritoneal dialysis can increase the risk of inguinal hernia or a recurrence thereof $[61,90,280]$.

The only pragmatic prevention for an inguinal hernia is smoking cessation and possibly not undertaking long-term and heavy physical work.

Known factors for the development of a recurrent inguinal hernia are: technique (see the next chapter), type of hernia (direct higher risk than indirect) and recurrent inguinal hernia (the more frequently a recurrence occurs, the higher the risk of a new recurrence).

Inguinal hernia is a known complication after radical retropubic prostatectomy, open procedure as well as endoscopic, and has been reported to occur in $7-21 \%$ of patients [6, 190, 287-289]. Even other types of lower midline incision surgery could promote the development of postoperative inguinal hernia [5, 289]. Urologists should be aware of this important postoperative complication and prophylactic surgical procedures must be evaluated to address the problem.

\section{Treatment of inguinal hernia}

Authors: Marc Miserez, Maarten Simons and Theo Aufenacker

What is the best technique for treating an inguinal hernia taking into account the type of hernia and the patient?

Search terms: RCT, hernia and specific names of the surgical techniques (46 combinations in total) in Medline, Cochrane library, references, correspondence and unpublished results. 


\section{Conclusions}

Level Operation techniques using mesh result in fewer 1A recurrences than techniques which do not use mesh.

Shouldice hernia repair technique is the best non-mesh repair method.

Endoscopic inguinal hernia techniques result in a lower incidence of wound infection, haematoma formation and an earlier return to normal activities or work than the Lichtenstein technique.

Endoscopic inguinal hernia techniques result in a longer operation time and a higher incidence of seroma than the Lichtenstein technique.

Level Mesh repair appears to reduce the chance of $1 \mathrm{~B}$ chronic pain rather than increase it. Endoscopic mesh techniques result in a lower chance of chronic pain/numbness than the Lichtenstein technique. In the long term (more than 3 to 4 years follow-up), these differences (non-meshendoscopic-Lichtenstein) seem to decrease for the aspect pain but not for numbness.

For recurrent hernias after conventional open repair, endoscopic inguinal hernia techniques result in less postoperative pain and faster reconvalescence than the Lichtenstein technique. Material-reduced meshes have some advantages with respect to long-term discomfort and foreign-body sensation in open hernia repair, but are possibly associated with an increased risk for hernia recurrence (possibly due to inadequate fixation and/or overlap) (Chap. 2.9).

From the perspective of the hospital, an open mesh procedure is the most cost-effective operation in primary unilateral hernias. From a socio-economic perspective, an endoscopic procedure is probably the most cost-effective approach for patients who participate in the labour market, especially for bilateral hernias. In cost-utility analyses including quality of life (QALYs), endoscopic techniques (TEP) may be preferable, since they cause less numbness and chronic pain (Chap. 2.18).

Level For endoscopic inguinal hernia techniques, 2A TAPP seems to be associated with higher rates of port-site hernias and visceral injuries, whilst there appear to be more conversions with TEP.
Level There appears to be a higher rate of rare but 2B serious complications with endoscopic repair, especially during the learning curve period.

Other open-mesh techniques: PHS, Kugel patch, plug and patch (mesh plug) and Hertra mesh (Trabucco), in short-term follow-up, result in comparable outcome (recurrence) to the Lichtenstein technique.

A young man (aged 18-30 years) with a lateral inguinal hernia has a risk of recurrence of at least $5 \%$ following a non-mesh operation and a long follow-up ( $>5$ years) (Chap. 2.8).

Level Endoscopic inguinal hernia techniques with a 2C small mesh $(\leq 8 \times 12 \mathrm{~cm})$ result in a higher incidence of recurrence compared with the Lichtenstein technique.

Women have a higher risk of recurrence (inguinal or femoral) than men following an open inguinal hernia operation due to a higher occurrence of femoral hernias (Chap. 2.7).

The learning curve for performing endoscopic inguinal hernia repair (especially TEP) is longer than for open Lichtenstein repair, and ranges between 50 and 100 procedures, with the first 30-50 being the most critical (Chap. 2.12).

For endoscopic techniques, adequate patient selection and training might minimise the risks for infrequent but serious complications in the learning curve (Chap. 2.12).

Level There does not seem to be a negative effect on

2C outcome when operated by a resident versus an attending surgeon (Chap. 2.12).

Specialist centres seem to perform better than general surgical units, especially for endoscopic repairs (Chap. 2.12).

Level All techniques (especially endoscopic techniques) 4 have a learning curve that is underestimated. For large scrotal (irreducible) inguinal hernias, after major lower abdominal surgery, and when no general anaesthesia is possible, the Lichtenstein repair is the preferred surgical technique.

For recurrent hernias, after previous posterior approach, an open anterior approach seems to have clear advantages, since another plane of dissection and mesh implantation is used. 
Stoppa repair is still the treatment of choice in case of complex hernias.

\section{Recommendations}

Grade All male adult ( $>30$ years) patients with a A symptomatic inguinal hernia should be operated on using a mesh technique.

When considering a non-mesh repair, the Shouldice technique should be used.

The open Lichtenstein and endoscopic inguinal hernia techniques are recommended as the best evidence-based options for the repair of a primary unilateral hernia, providing the surgeon is sufficiently experienced in the specific procedure.

For the repair of recurrent hernias after conventional open repair, endoscopic inguinal hernia techniques are recommended.

When only considering chronic pain, endoscopic surgery is superior to open mesh.

In inguinal hernia tension-free repair, synthetic non-absorbable flat meshes (or composite meshes with a non-absorbable component) should be used (Chap. 2.9).

The use of lightweight/material-reduced/largepore $(>1,000-\mu \mathrm{m})$ meshes can be considered in open inguinal hernia repair to decrease long-term discomfort, but possibly at the cost of increased recurrence rate (possibly due to inadequate fixation and/or overlap) (Chap. 2.9).

It is recommended that an endoscopic technique is considered if a quick postoperative recovery is particularly important (Chap. 2.14).

It is recommended that, from a hospital perspective, an open mesh procedure is used for the treatment of inguinal hernia (Chap. 2.18).

From a socio-economic perspective, an endoscopic procedure is proposed for the active working population, especially for bilateral hernias (Chap. 2.18).

Grade Other open-mesh techniques than Lichtenstein B (PHS, Kugel patch, plug and patch [mesh-plug] and Hertra mesh [Trabucco]) can be considered as an alternative treatment for open inguinal hernia repair, although only short-term results (recurrence) are available.

It is recommended that an extraperitoneal approach (TEP) is used for endoscopic inguinal hernia operations.
It is recommended that a mesh technique is used for inguinal hernia correction in young men (aged 18-30 years and irrespective of the type of inguinal hernia) (Chap. 2.8).

Grade (Endoscopic) hernia training with adequate C mentoring should be started with junior residents (Chap. 2.12).

Grade For large scrotal (irreducible) inguinal hernias, D after major lower abdominal surgery, and when no general anaesthesia is possible, the Lichtenstein repair is the preferred surgical technique.

In endoscopic repair, a mesh of at least $1 \times 15 \mathrm{~cm}$ should be considered.

It is recommended that an anterior approach is used in the case of a recurrent inguinal hernia which was treated with a posterior approach.

In female patients, the existence of a femoral hernia should be excluded in all cases of a hernia in the groin (Chap. 2.7).

A preperitoneal (endoscopic) approach should be considered in female hernia repair (Chap. 2.7).

All surgeons graduating as general surgeons should have a profound knowledge of the anterior and posterior preperitoneal anatomy of the inguinal region (Chap. 2.12).

Complex inguinal hernia surgery (multiple recurrences, chronic pain, mesh infection) should be performed by a hernia specialist (Chap. 2.12).

\section{Background}

Treatment of inguinal hernias An inguinal hernia is treated when acute complications occur (such as incarceration, strangulation and ileus), to reduce the symptoms and to prevent complications. The aim of treating an inguinal hernia is to reduce the symptoms by repairing the inguinal hernia with minimum discomfort for the patient and in the most cost-effective manner. Hernias can only be cured by surgical repair.

Conservative treatment Conservative management of inguinal hernias is discussed in Chap. 2.1. This chapter describes surgical treatment.

Surgical treatment The open surgical treatment of the inguinal hernia in adults consists of three elements: 
1. Dissection of the hernia sac from the spermatic cord structures.

2. Reduction of the hernia sac contents and resection or reduction of the hernia sac.

3. Repair and/or reinforcing of the fascial defect in the posterior wall of the inguinal canal.

An accurate dissection of the inguinal canal provides insight into the anatomy of the hernia. During the reduction, the content of the hernia sac is placed back into the peritoneal cavity. The peritoneal hernia sac is resected or reduced into the preperitoneal space.

The inguinal canal is restored by repairing the defect in the posterior wall by means of a so-called tissue suture technique or by covering the defect with synthetic material. Polypropylene mesh is usually the synthetic material of choice.

All of the tissue surgery techniques bear the name of the surgeon who promoted the method concerned (Marcy, Bassini, Halsted, McVay, Shouldice), as is also the case for the majority of prosthetic techniques with mesh (Lichtenstein, Stoppa, Wantz, Rutkow/Robbins), whereas, currently, often only the type of operation is stated (plug and patch, PHS, TEP, TAPP).

Techniques Conventional suturing technique (nonmesh) Bassini described the first rational hernia operation in 1884 but, unfortunately, his original operation was modified and corrupted. Not until 1950 was the modern version of the original Bassini procedure described by Shouldice, in which the posterior wall of the inguinal canal and the internal ring were repaired by means of sutures in several layers with a continuous non-soluble monofilament suture. Recent randomised research has shown that the Shouldice technique is considerably better than the nonoriginal Bassini technique and the Marcy technique (simple narrowing of the internal ring) with recurrence percentages in the long term of 15,33 and $34 \%$, respectively [36]. The Bassini technique and Marcy's technique are, therefore, obsolete.

The Shouldice technique is the best conventional treatment for primary inguinal hernia [279]. In experienced hands and specialised clinics, the results are very good (recurrence rates $0.7-1.7 \%$ ). In general practice, the results are less satisfying, with recurrence rates in the long term of $1.7-15 \%[36,279]$.

Mesh technique The approximation of tissues which do not normally lie against each other results in abnormal tension between these tissues. All classical sutured inguinal hernia operations share this factor-tension on the repair. This may result in ischaemia, which gives rise to pain, necrosis, tearing of sutures and a recurrent hernia. Fur- thermore, there are indications that some patients with inguinal hernias have an abnormal collagen metabolism, particularly in the elderly. The reinforcement of these tissues by synthetic material has become the established method. The concept of a tension-free repair of the defect had already emerged at the end of the 19th century, but a suitable biomaterial in the form of polypropylene mesh only became available in 1960 . The mesh material now most commonly used is a flat sheet of monopropylene.

The prosthetic repair of a defect in the posterior wall of the inguinal canal can be carried out in two fundamentally different manners. The defect is blocked with a plug or a larger, flat mesh prosthesis is placed over the fascia transversalis. Prostheses can be inserted into the groin anteriorly via an inguinal incision or posteriorly in the preperitoneal space via a classic open approach or along the endoscopic route.

Mesh: anterior open approach Tension-free repair of inguinal hernia has been strongly promoted since 1984 by Lichtenstein [183]. Via an inguinal incision, preferably under local anaesthetic, the polypropylene mesh is sutured to the posterior wall of the inguinal canal with considerable overlap. The mesh is positioned between the internal oblique muscle and the aponeurosis of the external oblique and is sutured to the inguinal ligament. Crucial is the adequate overlap of the posterior wall of the inguinal canal, especially $2 \mathrm{~cm}$ medial to the pubic tubercle, although a very low risk routine exploration of the femoral canal is advised, especially in the absence of an inguinal hernia and women. Different meshes or other devices were developed: mesh-plug (plug placed deep into the inguinal ring/medial defect, mesh placed on the posterior wall of the inguinal canal), PHS (device covering three spaces: preperitoneal space, deep inguinal ring/medial defect, posterior wall of the inguinal canal), Hertra sutureless mesh (Trabucco). Rives used a transinguinal approach to place the mesh preperitonealy.

Mesh: posterior open approach The posterior approach to the entire myopectineal orifice of Fruchaud via an abdominal incision with the insertion of a large prosthesis completely overlapping all orifices has been popularised by Stoppa since 1980 [286]. Goss and Mahorner (1962) were the first to come up with the idea, and Stoppa (for bilateral recurrent inguinal hernias) and Wantz developed it for unilateral inguinal hernia [256]. The Stoppa technique is still the treatment of choice in the case of complex hernias (bilateral and several recurrences) [35]. Another technique was developed using a specific mesh type (Kugel). Kugel preperitoneal open mesh placement in the short term provides results comparable to the Lichtenstein technique [83, 167]. 
Mesh: posterior endoscopic approach Since 1990, the Stoppa technique has been performed endoscopically, by means of both the transperitoneal (TAPP) and preperitoneal (TEP) approaches [186].

Just as 100 years ago, many of these new techniques have been modified and corrupted. In 2007, there are countless variants concerning the approach, technique and prosthetic material, with comparable short-term results.

Theoretical considerations Theoretically, Lichtenstein mesh is on the wrong side of the hernia defect. The preperitoneal insertion of a large mesh which seals off the entire myopectineal orifice of Fruchaud from the inside would, therefore, in theory, seem to be the best treatment for inguinal hernia. The tensions which have caused the hernia keep the mesh in place, in accordance with Pascal's law. Furthermore, if the operation can take place by means of a minimally invasive (endoscopic) method, the ideal operation would seem to be a reality.

In the case of recurrent hernias, a new, previously unused approach is preferable to the previous route. In order to place a prosthesis well, an ample dissection is required. Reoperation via an inguinal incision increases the risk of haemorrhage and wound infection, damage to cutaneous nerves or damage to the spermatic cord. When a recurrence occurs after an operation via an inguinal incision, reoperation via the posterior preperitoneal approach is preferable. The opposite is true for recurrent hernias after abdominal or endoscopic preperitoneal operations. Then, an inguinal approach is safer and easier. For bilateral hernias, and certainly if a (bilateral) recurrence is involved, a posterior (endoscopic) preperitoneal approach is preferred.

The evolution in the treatment of inguinal hernia from the Bassini technique to the open mesh and endoscopic techniques has led to more than 100 randomised studies in which an attempt has been made to establish the most efficient and effective treatment technique.

\section{Literature study}

Search terms: RCT, hernia and specific names of the surgical techniques (46 combinations in total) in Medline, Cochrane library, references, correspondence and unpublished results. The results were published in the British Journal of Surgery, the Annals of Surgery, the Cochrane Library, Surgical Endoscopy, Hernia etc.

Systematic reviews and a meta-analysis were carried out by the EU Hernia Trialists Collaboration concerning the risk of recurrences, complications, postoperative recovery, grade of difficulty (learning curve) and costs [70-72, 115, 116, 197, 275, 304, 305].
All of the following factors need to be considered when choosing a treatment [171]:

- Risk of recurrence

- Safety (risk of complications)

- Postoperative recovery and quality of life (resumption of work)

- Grade of difficulty and reproducibility (learning curve)

- Costs (hospital and societal costs)

Results from the literature concerning techniques for inguinal hernia repair The Shouldice technique is the best non-mesh repair for primary inguinal hernia [279]. The Lichtenstein technique, introduced in 1984, is currently the best evaluated and most popular of the different open-mesh techniques: it is reproducible with minimal perioperative morbidity, it can be performed in day care (under local anaesthesia) and has low recurrence rates $(\leq 4 \%)$ in the long term $[17,183]$.

Mesh or non-mesh? A systematic review of RCTs by the Cochrane Collaboration/EU Hernia Trialists Collaboration in 2002 and 2003 showed strong evidence that fewer hernias recur after mesh repair than following nonmesh repair, with a separate analysis for the Shouldice repair. Mesh appears to reduce the chance of chronic pain rather than increase it $[41,197,275]$. Bittner stated that there was no difference in the recurrence rate for the Shouldice repair versus endoscopic techniques, in contrast to other suture repairs that were clearly inferior to endoscopic techniques with respect to the recurrence rate [41]. The incidence of chronic groin pain was clearly lower in the endoscopic techniques versus Shouldice (2.2 vs. $5.4 \% ; P<0.00007)$ and other non-mesh repairs $(3.9$ vs. $9.0 \% ; P<0.00001)$.

Since then, three RCTs comparing the Shouldice and Lichtenstein techniques have been published [54, 205, 220]. One additional trial compared open non-mesh and Lichtenstein techniques and reported the results on recurrence with a more than 10-year follow-up period [309]. Recurrence rates were clearly higher after the Shouldice procedure in those four trials, except for the trial by Miedema et al. In this latter trial, where the surgery was performed by first- and second-year residents under the supervision of an experienced general surgeon, the rate of severe chronic pain was also clearly increased in the mesh group.

After conventional repair, recurrences can be expected to occur several years postoperatively and increase with a prolonged follow-up. With various mesh techniques, a recurrence is frequently demonstrated early in the follow- 
up due to technical failure. It is not known whether the incidence of chronic pain might decrease with longer follow-up. To determine the results in the long term, we performed an additional meta-analysis comparing the Shouldice repair with different mesh techniques in all trials with a follow-up of more than 3 years (Table 3 ).

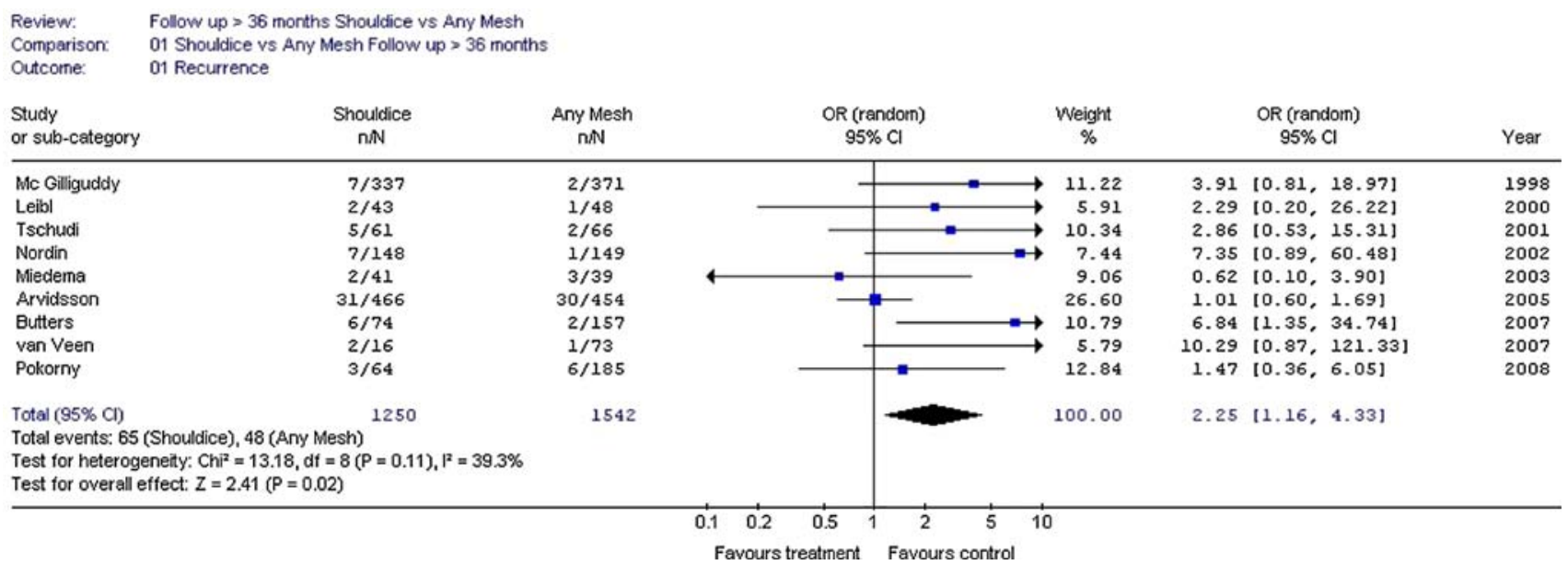

Fig. 1

Table 3 Long-term follow-up (>36 months) of RCTs comparing Shouldice with different mesh techniques

\begin{tabular}{|c|c|c|c|c|c|c|c|}
\hline Year & First author & Groups & $\begin{array}{l}\text { Number of } \\
\text { patients }\end{array}$ & $\begin{array}{l}\text { Follow-up duration } \\
\text { (months, mean) }\end{array}$ & $\begin{array}{l}\text { Follow-up number } \\
\text { (percentage with physical } \\
\text { examination) }\end{array}$ & $\begin{array}{l}\text { Recurrence } \\
(\%)\end{array}$ & $\begin{array}{l}\text { Chronic } \\
\text { pain }(\%)^{\mathrm{a}}\end{array}$ \\
\hline 1998 & $\begin{array}{l}\text { McGilliguddy } \\
\text { [201] }\end{array}$ & $\begin{array}{l}\text { Lichtenstein vs. } \\
\text { Shouldice }\end{array}$ & $708^{\mathrm{b}}$ & 60 & $476^{\mathrm{b}}(67 \%)$ & 0.5 vs. 2.1 & 1.1 vs. 0.3 \\
\hline 2000 & $\begin{array}{l}\text { Leibl et al. } \\
\text { [182] }\end{array}$ & TAPP vs. Shouldice & 102 & 70 & Probably $91(89.2 \%)$ & 2.1 vs. 4.7 & 0 vs. 0 \\
\hline 2001 & $\begin{array}{l}\text { Tschudi et al. } \\
\text { [300] }\end{array}$ & TAPP vs. Shouldice & $127^{\mathrm{b}}$ & 60 & $107^{\mathrm{b}}(84 \%)$ & 3.0 vs. 8.2 & 1.5 vs. 14.8 \\
\hline 2002 & $\begin{array}{l}\text { Nordin et al. } \\
\text { [220] }\end{array}$ & $\begin{array}{l}\text { Lichtenstein vs. } \\
\text { Shouldice }\end{array}$ & 297 & 36 & $284(96 \%)$ & 0.7 vs. 4.7 & 5.6 vs. 4.2 \\
\hline 2004 & $\begin{array}{l}\text { Miedema et al. } \\
\text { [205] }\end{array}$ & $\begin{array}{l}\text { Lichtenstein vs. } \\
\text { Shouldice }\end{array}$ & 101 & 85 & $50(50 \%)$ & 7.7 vs. 4.9 & 37.9 vs. 7.1 \\
\hline 2004 & $\begin{array}{l}\text { Köninger et al. } \\
\text { [165] }\end{array}$ & $\begin{array}{l}\text { TAPP-Lichtenstein vs. } \\
\text { Shouldice }\end{array}$ & 280 & 52 & $231(83 \%)$ & - & $\begin{array}{c}24.2 \text { vs. } \\
37.8\end{array}$ \\
\hline 2005 & $\begin{array}{l}\text { Arvidsson } \\
\text { et al. [21] }\end{array}$ & TAPP vs. Shouldice & 1,068 & 61 & $920(86 \%)$ & 6.6 vs. 6.7 & - \\
\hline 2007 & $\begin{array}{l}\text { Butters et al. } \\
\text { [54] }\end{array}$ & $\begin{array}{l}\text { TAPP-Lichtenstein vs. } \\
\text { Shouldice }\end{array}$ & 280 & 52 & $231(83 \%)$ & 1.3 vs. 8.1 & - \\
\hline 2007 & $\begin{array}{l}\text { Berndsen et al. } \\
\text { [38] }\end{array}$ & TAPP vs. Shouldice & 1,068 & 60 & $867(81 \%)$ & - & 8.5 vs. 11.4 \\
\hline 2007 & $\begin{array}{l}\text { van Veen et al. } \\
\text { [309] }\end{array}$ & $\begin{array}{l}\text { Lichtenstein vs. } \\
\text { Shouldice }\end{array}$ & 182 & 128 & $80(44 \%)$ & 1.4 vs. 12.5 & - \\
\hline 2008 & $\begin{array}{l}\text { Pokorny et al. } \\
\text { [240] }\end{array}$ & $\begin{array}{l}\text { TEP/TAPP/Lichtenstein } \\
\text { vs. Shouldice }\end{array}$ & 272 & 36 & $249(92 \%)$ & 3.3 vs. 4.7 & 5.4 vs. 6.3 \\
\hline
\end{tabular}

${ }^{a}$ Variety of definitions, including any pain

b No. of hernias 
When performing a meta-analysis on the data (see figures) with $>3$ years follow-up, a random analysis is used because of the clinical and methodological diversity. The Shouldice technique performs significantly worse regarding the recurrence odds ratio (OR) of 1.99 (95\% confidence interval [CI]: 1.05-3.79), but it does not significantly differ compared to mesh techniques regarding moderate and severe pain OR 1.16 (95\% CI: 0.44-3.02).
Significant advantages for Lichtenstein included shorter operation time (by 8-13 $\mathrm{min}$ ), lower incidence of seroma and recurrences. The latter was strongly influenced by the Veterans Affairs (VA) Multicenter Trial, where the minimum mesh size in endoscopic surgery was $7.6 \times 15 \mathrm{~cm}$ (see below) [215]. When this study is excluded, there is no difference in the recurrence rates between open and endoscopic surgery.

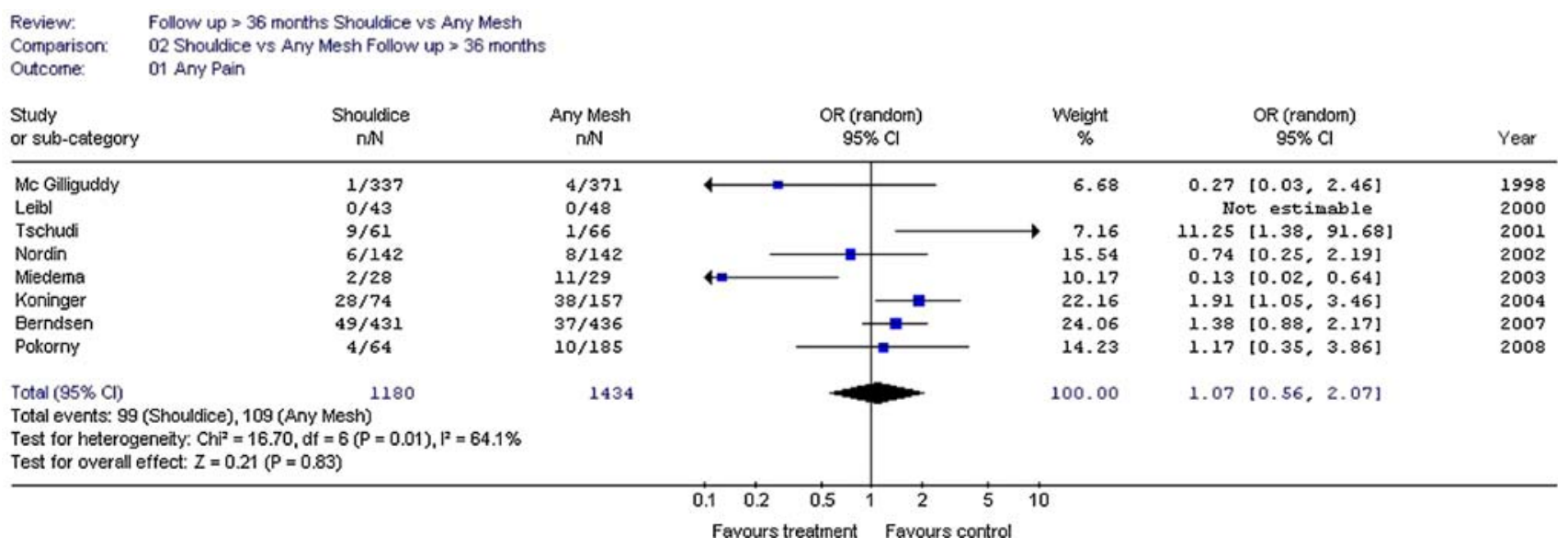

Fig. 2

Above data demonstrates that a mesh technique is superior regarding recurrence but not at the expense of more pain.

Open mesh versus endoscopic mesh Two recent metaanalyses of RCTs were published in 2005 and compare open and endoscopic mesh techniques and include all relevant papers up to April 2004, including the large Veterans Affairs Multicenter Trial by Neumayer et al. [198, 272]. Schmedt made a specific comparison between endoscopic procedures (TAPP and TEP) and only Lichtenstein as the open mesh technique.

Significant advantages for endoscopy include lower incidence of wound infection, haematoma and chronic pain/numbness, with earlier return to normal activities or work (6 days). The McCormack review found heterogeneity among RCTs in the length of hospital stay. There were greater differences in the mean length of stay between different hospitals than between different operative techniques, possibly reflecting differences in health care systems versus differences due to types of endoscopic repair. An earlier meta-analysis (possibly outdated) had shown a small $(3.4 \mathrm{~h})$ decrease in hospital stay in favour of endoscopic repair [203]. A very recent systematic review comparing open mesh and suture repair versus endoscopic TEP also showed a shorter hospital stay in 6/11 trials [168].
There also appears to be a higher rate of rare but serious complications with respect to major vascular and visceral (especially bladder) with the endoscopic approach. Most of these lesions were seen with TAPP (0.65 vs. $0-0.17 \%$ for TEP and open mesh repair). The transabdominal route of TAPP might also cause more adhesions, leading to intestinal obstruction in a small number of cases [199]. In a separate evaluation of potentially lethal complications, the investigators conclude that no significant differences were found, but a definitive statistical evaluation was not possible due to the low incidence of these complications. A specific meta-analysis comparing TAPP versus TEP (including eight non-randomised studies) states that there is insufficient data to allow conclusions to be drawn, but suggests that, indeed, TAPP is associated with higher rates of port-site hernias and visceral injuries, whilst there appear to be more conversions with TEP [272]. Additional recent publications of RCTs comparing TEP versus Lichtenstein confirm the data from the two meta-analyses, except for the shorter operation time with Lichtenstein [87, 176].

The best investigated anterior approach is the Lichtenstein repair and the best posterior is the endoscopic repair. For same reason as mentioned above, we performed an additional meta-analysis of long-term follow-up concerning pain and recurrence. Since many trials publish 
Table 4 Long-term follow-up (>48 months) of RCTs comparing endoscopic mesh techniques (TEP/TAPP) with Lichtenstein mesh repair

\begin{tabular}{|c|c|c|c|c|c|c|c|}
\hline Year & First author & Groups & $\begin{array}{l}\text { Number } \\
\text { of } \\
\text { patients }\end{array}$ & $\begin{array}{l}\text { Follow-up } \\
\text { duration } \\
\text { (months, } \\
\text { mean) }\end{array}$ & $\begin{array}{l}\text { Follow-up number } \\
\text { (percentage with } \\
\text { physical examination) }\end{array}$ & $\begin{array}{l}\text { Recurrence } \\
(\%)\end{array}$ & $\begin{array}{l}\text { Chronic } \\
\text { pain }(\%)^{\text {a }}\end{array}$ \\
\hline 2002 & Wright et al. [321] & TEP vs. Lichtenstein & 256 & 60 & $256(48 \%)$ & 2.0 vs. 0 & $\begin{array}{l}\text { Impossible to } \\
\text { extract the } \\
\text { data }\end{array}$ \\
\hline 2003 & Douek et al. [84] & TAPP vs. Lichtenstein & 403 & 69 & $242(100 \%)$ & 1.6 vs. 2.5 & 0 vs. 5.0 \\
\hline 2004 & Heikinnen et al. [130] & $\begin{array}{l}\text { TAPP/TEP }{ }^{\mathrm{b}} \text { vs. } \\
\text { Lichtenstein }^{\mathrm{b}}\end{array}$ & 123 & 70 & $121(75 \%)$ & 8.1 vs. 3.4 & 0 vs. 6.8 \\
\hline 2004 & Grant et al. [117] & TEP vs. Lichtenstein & 928 & 60 & $558(0 \%)^{\mathrm{c}}$ & Data not available & 2.1 vs. 1.5 \\
\hline 2004 & Köninger et al. [165] & TAPP vs. Lichtenstein & 187 & 52 & $157(100 \%)$ & Data not available & 0 vs. 3.9 \\
\hline 2007 & Butters et al. [54] & TAPP vs. Lichtenstein & 187 & 52 & $157(100 \%)$ & 1.2 vs. 1.3 & $\begin{array}{l}\text { Impossible to } \\
\text { extract the } \\
\text { data }\end{array}$ \\
\hline 2008 & Hallén et al. [124] & TEP vs. Lichtenstein & 168 & 88 & $147(100 \%)$ & 4.3 vs. 5.1 & 5.5 vs. 2.5 \\
\hline 2007 & Eklund et al. [88] & TAPP vs. Lichtenstein ${ }^{\mathrm{d}}$ & 147 & 61 & $132(100 \%)$ & 19 vs. 18 & 0 vs. 0 \\
\hline
\end{tabular}

a Variety of definitions, but only severe pain prevalence was scored

b Three separate trials combined

c Only questionnaire

${ }^{d}$ Only recurrent hernia

short-term results about pain and because the prevalence of pain diminishes after a longer time period, the best comparison between the two techniques mentioned is with long-term follow-up. Therefore, Table 4 demonstrates the data of all trials with a follow-up of over 48 months.

When performing a meta-analysis (see figures) on the data with a minimum of 4 years follow-up, a random analysis is used because of the clinical and methodological diversity. The Lichtenstein technique performs slightly but not significantly better concerning the recurrence OR of 1.16 (95\% CI: 0.63-2.16), but does have a non-significant trend towards more severe pain OR of 0.48 (95\% CI: $0.11-$ 2.06).

The difficulty in the pain area is, of course, the large variation in definitions and, therefore, any firm statement regarding this topic remains difficult.

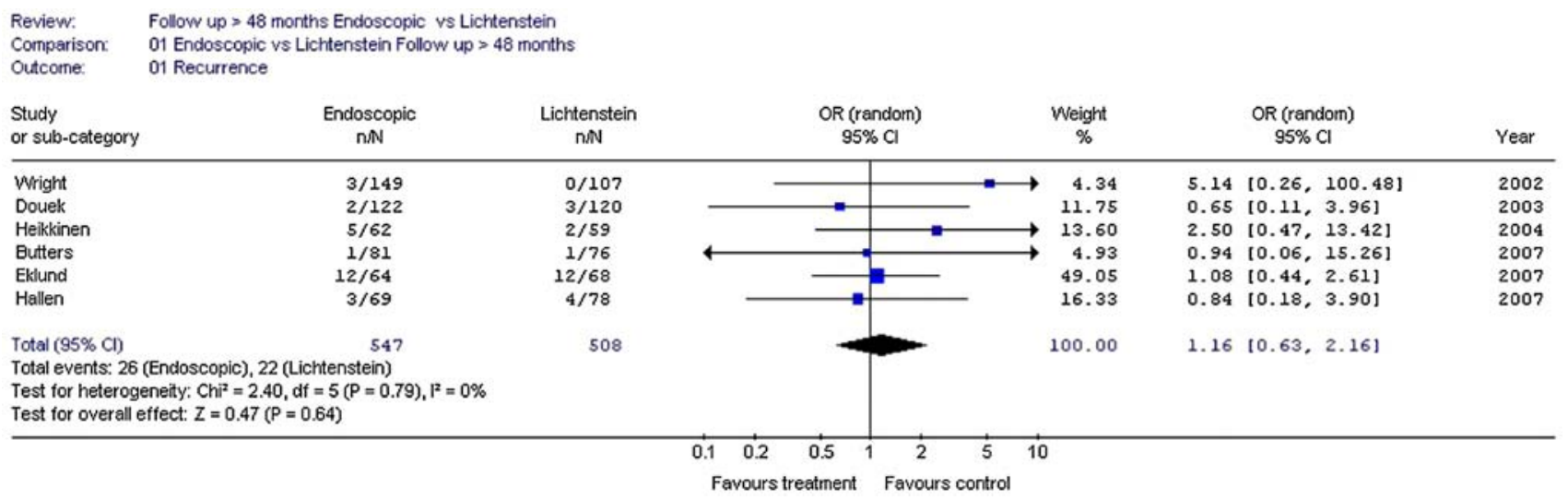

Fig. 3 


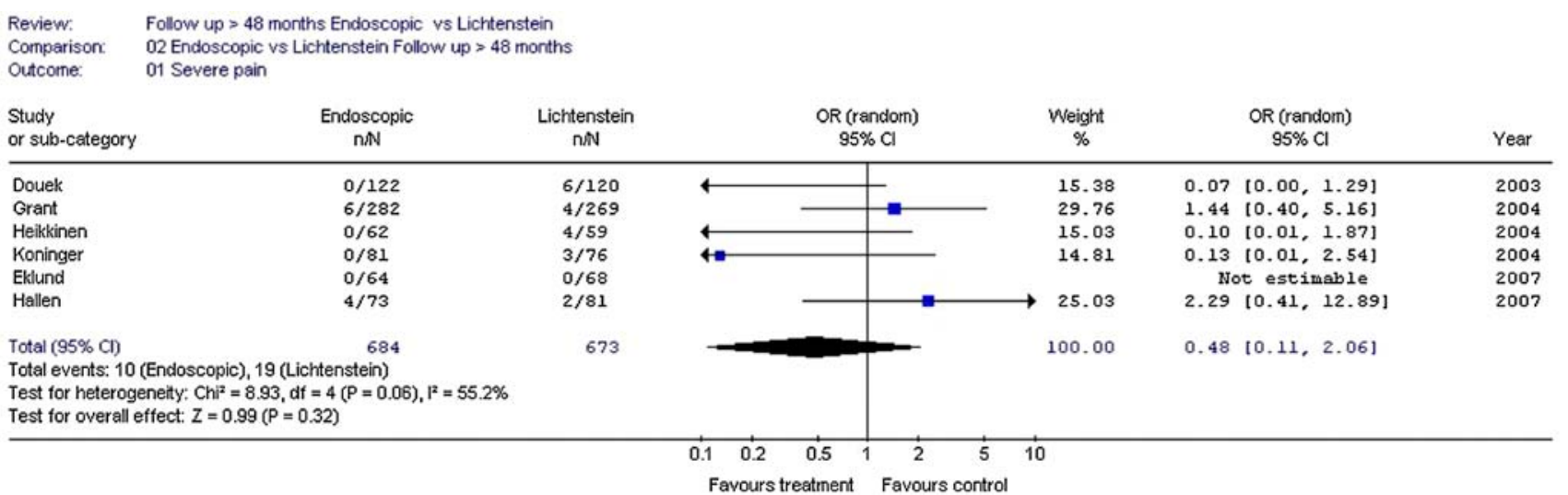

Fig. 4

These data seem to confirm the comparable recurrence rates in the long term for both open and endoscopic mesh repairs. In addition, the incidence of (severe) chronic pain between both groups seems to equalise with time. Only numbness seems to persist $[54,115]$.

Long-term follow-up (>48 months) of RCTs comparing endoscopic mesh techniques (TEP/TAPP) with Lichtenstein repair:

Table 5 Outcome parameter: numbness (\%)

\begin{tabular}{llll}
\hline 2003 & Douek et al. & TAPP vs. Lichtenstein & 0 vs. 14.5 \\
2004 & Grant et al. & TEP vs. Lichtenstein & 12.7 vs. 24.7 \\
2007 & Butters et al. & TAPP vs. Lichtenstein & 0 vs. 10 \\
2008 & Hallén et al. & TEP vs. Lichtenstein & 12.3 vs. 32.1 \\
\hline
\end{tabular}

When a mesh-based repair is chosen, the best approach to the groin is under debate. This is mainly caused by discussion about recurrence on one hand and chronic pain on the other.

With adequate surgical technique and training, the recurrence rate (after endoscopic operations) can be reduced significantly. The higher recurrence rate for the endoscopic repair in some papers (compared with the other publications) might be related to the size of the mesh used, which is currently considered to be too small: the $8-\mathrm{cm}$ minimum height of the mesh in the VA Multicenter Trial or a mesh size of $7 \times 12 \mathrm{~cm} \mathrm{[20,215].} \mathrm{A} \mathrm{recent} \mathrm{publication}$ of a multicentric trial in France with more than 300 patients and a follow-up period of more than 2 years also showed higher recurrence rates with endoscopic repair (especially for direct hernias: 27.3 vs. $6.5 \%$ for Shouldice repair performed in $90 \%$ of cases); in $69 \%$ of the patients treated endoscopically, a mesh of dimensions $\leq 8 \times 12 \mathrm{~cm}$ was used [206].

Results of non-Lichtenstein open-mesh techniques The small studies (short follow-up) describing the use of these methods provided comparable results for recurrence to the Lichtenstein technique [7, 42, 103, 154, 155, 217, 270]. Longer follow-up data on recurrence/chronic pain are missing at the present time.

RCTs concerning non-Lichtenstein mesh repairs:

Table 6

\begin{tabular}{|c|c|c|c|c|c|c|c|}
\hline $\begin{array}{l}\text { Year of } \\
\text { publication }\end{array}$ & First author & Groups & $\begin{array}{l}\text { Number of } \\
\text { patients }\end{array}$ & $\begin{array}{l}\text { Follow-up } \\
\text { duration (months) }\end{array}$ & $\begin{array}{l}\text { Follow-up number } \\
\text { (percentage with } \\
\text { physical examination) }\end{array}$ & $\begin{array}{l}\text { Recurrence } \\
(\%)\end{array}$ & $\begin{array}{l}\text { Chronic } \\
\text { pain }(\%)\end{array}$ \\
\hline 2000 & Kingsnorth et al. [154] & $\begin{array}{l}\text { Mesh-plug } \\
\text { vs. } \\
\text { Lichtenstein }\end{array}$ & $\begin{array}{l}141 \\
68 / 73\end{array}$ & 14 days & $100 \%$ & No data & No data \\
\hline 2002 & Kingsnorth et al. [155] & $\begin{array}{l}\text { PHS } \\
\text { vs. } \\
\text { Lichtenstein }\end{array}$ & $\begin{array}{l}206 \\
103 / 103\end{array}$ & 12 & $98 \%$ & $\begin{array}{l}0 \% \text { PHS } \\
2 \% \text { Lichtenstein }\end{array}$ & No data \\
\hline 2005 & Nienhuijs et al. [218] & $\begin{array}{l}\text { PHS } \\
\text { vs. } \\
\text { Lichtenstein } \\
\text { vs. } \\
\text { Mesh-plug }\end{array}$ & $\begin{array}{l}334 \\
111 / 110 / 143\end{array}$ & 15 & $95.8 \%$ & $\begin{array}{l}2.5 \% \\
\text { No differences }\end{array}$ & $\begin{array}{l}43.3 \% \\
\text { No differences }\end{array}$ \\
\hline
\end{tabular}


Table 6 continued

\begin{tabular}{|c|c|c|c|c|c|c|c|}
\hline $\begin{array}{l}\text { Year of } \\
\text { publication }\end{array}$ & First author & Groups & $\begin{array}{l}\text { Number of } \\
\text { patients }\end{array}$ & $\begin{array}{l}\text { Follow-up } \\
\text { duration (months) }\end{array}$ & $\begin{array}{l}\text { Follow-up number } \\
\text { (percentage with } \\
\text { physical examination) }\end{array}$ & $\begin{array}{l}\text { Recurrence } \\
(\%)\end{array}$ & $\begin{array}{l}\text { Chronic } \\
\text { pain }(\%)\end{array}$ \\
\hline 2006 & Dogru et al. [83] & $\begin{array}{l}\text { Kugel } \\
\text { vs. } \\
\text { Lichtenstein }\end{array}$ & $\begin{array}{l}140 \\
70 / 70\end{array}$ & 24 & $99 \%$ & $\begin{array}{l}0 \% \text { Kugel } \\
1.4 \% \text { Lich }\end{array}$ & No data \\
\hline 2006 & Sanjay et al. [270] & $\begin{array}{l}\text { PHS } \\
\text { vs. } \\
\text { Lichtenstein }\end{array}$ & $\begin{array}{l}64 \\
31 / 33\end{array}$ & 6 weeks & $94 \%$ & $\begin{array}{l}3 \% \text { PHS } \\
0 \% \text { lich }\end{array}$ & No data \\
\hline 2007 & Adamonis et al. [7] & $\begin{array}{l}\text { Trabucco } \\
\text { vs. } \\
\text { Mesh-plug }\end{array}$ & $\begin{array}{l}100 \\
50 / 50\end{array}$ & 21 & $57 \%$ & $\begin{array}{l}4 \% \text { Trab } \\
4 \% \text { M-P }\end{array}$ & $\begin{array}{l}30 \% \text { Trab } \\
19 \% \text { M-P }\end{array}$ \\
\hline 2007 & Frey et al. [103] & $\begin{array}{l}\text { Mesh-plug } \\
\text { vs. } \\
\text { Lichtenstein }\end{array}$ & $\begin{array}{l}597 \\
297 / 298\end{array}$ & 12 & $85.3 \%$ & $\begin{array}{l}0.3 \% \mathrm{M}-\mathrm{P} \\
0 \% \text { Lich }\end{array}$ & $\begin{array}{l}14.2 \% \text { Lich } \\
7 \% \text { M-P }\end{array}$ \\
\hline
\end{tabular}

For bilateral hernias, the meta-analyses comparing endoscopic versus open surgery are based on few data; there is limited evidence showing no significant difference in persisting pain (TEP vs. open mesh) or recurrence (TEP and TAPP vs. open mesh); there is limited evidence to suggest that TAPP reduces the time taken to return to normal activities compared with open-mesh repair. In an RCT comparing TAPP versus Lichtenstein for bilateral and recurrent hernias, three quarters of the patients with a recurrence after endoscopic repair had bilateral hernias treated with one large mesh $(30 \times 8 \mathrm{~cm})$ [195]. Thus, in bilateral hernias, a sufficiently large mesh should be used or two different meshes (e.g. $15 \times 13 \mathrm{~cm}$ on both sides).

For recurrent hernias, the endoscopic approach after previous open repair (and vice versa) seems to have clear advantages, since another plane of dissection and mesh implantation is used. In an RCT comparing TEP versus TAPP versus Lichtenstein after previous conventional open repair, the endoscopic approach significantly increased the operative time (only TEP) but reduced perioperative complications, postoperative pain, analgesic requirement and time to return to normal activities [78]. Another study comparing TAPP and Lichtenstein showed less postoperative pain and shorter sick leave for the endoscopic group [88]. The recurrence rate in both groups after 5 years was $18-19 \%$ (94\% FU) and also the incidence of chronic pain was comparable (although a lack of congruent definitions was reported and the size of the mesh in endoscopic repair of $7 \times 12 \mathrm{~cm}$ is currently considered to be too small).

For large scrotal (irreducible) inguinal hernias, after major lower abdominal surgery, previous radiotherapy of pelvic organs and when no general anaesthesia is possible, the Lichtenstein repair is the generally accepted treatment.
For any male patient treated with a large preperitoneal mesh, future prostatic surgery might be more problematic. Therefore, it is suggested that a rectal examination and PSA screening should be considered in all male patients between 40 and 70 years of age before proceeding to a preperitoneal mesh placement [138].

In the future, more detailed long-term evaluation with further well-structured and adequately powered RCTs with improved standardisation of hernia type, operative technique and surgeons' experience and the definition of major endpoints is necessary.

\section{Inguinal hernia in women}

Authors: Joachim Conze and Morten Bay Nielsen

Following a non-mesh inguinal hernia operation, is the risk of recurrence lower for women than for men? Should women be treated with a different strategy?

Search terms: inguinal hernia, treatment, women, female.

\section{Conclusions}

Level Women have a higher risk of recurrence 2C (inguinal or femoral) than men following an open inguinal hernia operation due to a higher occurrence of femoral hernias.

\section{Recommendations}

Grade In female patients, the existence of a femoral D hernia should be excluded in all cases of a hernia in the groin. 
A preperitoneal (endoscopic) approach should be considered in female hernia repair.

Women account for $8-9 \%$ of all inguinal and femoral hernia operations performed. In subgroup analyses from many studies, the recurrence rate of a non-mesh inguinal hernia operation in women seems to be comparable to that after type I and type II (EHS) inguinal hernia operations in men $(2-13 \%)$, dependent on the follow-up duration [85, $106,134,259]$. In epidemiological studies from national databases, reoperation rates after female herniorrhaphy are higher, compared to males, without any difference between anterior mesh and non-mesh repairs [30, 162]. In approximately $40 \%$ of reoperations, a femoral recurrence is found. It is not known whether these femoral 'recurrences' represent hernias overlooked at the primary operation or de novo hernias. The high frequency of femoral recurrence after inguinal herniorrhaphy in women argues for the use of endoscopic repair, covering both the inguinal and femoral orifices simultaneously.

\section{Lateral inguinal hernia in young men (aged 18-30 years)}

Authors: Morten Bay Nielsen and Joachim Conze

Does a young man have a very low risk of recurrence following a non-mesh inguinal hernia operation due to an indirect hernia? Is mesh treatment indicated for this category of patients?

Search terms: inguinal hernia, treatment.

\section{Conclusions}

Level A young man (aged 18-30 years) with a lateral 2B inguinal hernia has a risk of recurrence of at least 5\% following a non-mesh operation and a long follow-up ( $>5$ years).

\section{Recommendations}

Grade It is recommended that a mesh technique is used B for inguinal hernia correction in young men (1830 years of age and irrespective of the type of inguinal hernia).

In view of the discussion concerning the risk of recurrence in young men following a non-mesh inguinal hernia operation due to a lateral inguinal hernia and the concern for fertility issues, this category deserves to be considered in a separate chapter. About $5 \%$ of all inguinal hernia operations are performed on men between the ages of 18 and 30 years. Indirect inguinal hernias account for the majority of these operations. From studies (level 3 and 4), it is known that after 2 to 5 years of follow-up, the risk of recurrence after Shouldice repair is 1-3\% lower than for an operation due to a direct inguinal hernia. For this category of patients, Friis and Lindahl compared a Lichtenstein and an annuloraphy and saw recurrence rates of 0 and $2.2 \%$, respectively, with 2 years of follow-up after the repair of a primary hernia [105]. In a randomised study after a followup of more than 10 years, Beets et al. described recurrence rates of more than $30 \%$ for an indirect inguinal hernia after both an annuloraphy as well as a modified Bassini technique [36]. In a retrospective series of more than 1,000 annuloraphies for a lateral inguinal hernia, the recurrence rate rose to $18 \%$ after a follow-up of 10 years [145]. Analysis of data from the Danish Hernia Database shows a reoperation rate almost twice as high after non-mesh repairs compared to Lichtenstein and other open mesh repairs in males $<30$ years of age, operated for a primary indirect hernia (unpublished data).

In a questionnaire study, in patients below 55 years of age operated for an indirect hernia, no relevant difference in chronic pain was found between patients operated with mesh and non-mesh techniques and no study has shown specific mesh-related problems in this subgroup of patients [33]. In summary, there is no evidence to support a nonmesh approach in this subgroup of patients.

\section{Biomaterials}

Authors: Jan Kukleta and Joachim Conze

What mesh type is the most suitable in inguinal hernia repair, and what mesh-related complications can occur?

Search terms: mesh, biomaterial, inguinal hernia, mesh complications.

\section{Conclusions}

Level Operation techniques using mesh result in 1A fewer recurrences than techniques which do not use mesh.

Level Material-reduced meshes have some advantages 1B with respect to long-term discomfort and foreign-body sensation in open hernia repair, but are possibly associated with an increased risk for hernia recurrence (possibly due to inadequate fixation and/or overlap).

\section{Recommendations}

Grade In inguinal hernia tension-free repair, synthetic A non-absorbable flat meshes (or composite meshes with a non-absorbable component) should be used. 
The use of lightweight/material-reduced/largepore $(>1,000-\mu \mathrm{m})$ meshes in open inguinal hernia repair can be considered to decrease long-term discomfort, but possibly at the cost of increased recurrence rate (possibly due to inadequate fixation and/or overlap).

The use of synthetic mesh substantially reduces the risk of hernia recurrence irrespective of the placement method. Mesh repair appears to reduce the chance of persisting pain rather than increase it [72].

Only non-absorbable meshes or composite meshes with a non-absorbable component should be used in inguinal hernia repair in adults.

There is a great variety of meshes available differing in textile parameters (polymer, filament, construction, pore size, elasticity, tensile strength, weight, surface). We do not know the parameters of the ideal mesh. The use of mesh can be related to some non-specific complications (pain, infection, recurrence) and some specific complications (shrinkage, dislocation, migration, erosion). In open inguinal hernia repair, the use of a monofilament polypropylene mesh is advised to reduce the chance of incurable chronic sinus formation or fistula which can occur in patients with a deep infection. The chance of complete wound healing after adequate drainage is virtually impossible when a multifilament mesh is used as bacteria $(\varnothing 1 \mu \mathrm{m})$ can hide from the leucocytes $(\varnothing$ $>10 \mu \mathrm{m})$ because the mesh has a closer weave structure with a smaller pore diameter $(\varnothing 10 \mu \mathrm{m})$ and the mesh cannot be 'sterilised.' [293].

Weight-reduced mesh materials $(>1,000 \mu \mathrm{m})$, macroporous and oligofilament structures seem to shrink less, cause less inflammatory reaction and induce less extensive scar-tissue formation and are, therefore, more likely to be integrated with less long-term discomfort and foreign-body sensation when implanted in open hernia repair [47-50, $131,137,171,172]$ but, possibly, they are associated with an increased risk for hernia recurrence [50, 137, 153, 228, 244] in high-risk conditions (large direct hernia), if the mesh is not adequately fixed and/or overlapping.

There is not sufficient data on sexual dysfunction in relation to the variable properties of different prosthetic materials or different surgical techniques used.

\section{Day surgery}

Authors: Maciej Smietanski and Rene Fortelny

Can inguinal hernia surgery be performed in a day surgery setting? Is this safe and cost-effective?

Search terms: (groin or inguinal) hernia, ambulatory, day surgery, random* in PubMed; function: related articles in PubMed; literature lists of relevant articles.

\section{Conclusions}

Level Inguinal hernia surgery as day surgery is as safe 2B and effective as that in an inpatient setting, and more cost-effective.

Level Inguinal hernia surgery can easily be performed 3 as day surgery, irrespective of the technique used. Selected older and ASA III/IV patients are also eligible for day surgery.

\section{Recommendations}

Grade An operation in day surgery should be considered B for every patient.

Day surgery is an admission to a unit for a diagnostic or therapeutic treatment by a medical specialist, in which discharge takes place on the same day after a period of recovery under (para)medical supervision [111]. An inguinal hernia repair performedintheoutpatientdepartmentunderlocalanaesthetic, in which the patient goes home shortly after the intervention, is considered to be day surgery.

As early as 1955, the advantages of inguinal hernia repair as day surgery were already described in the literature: quicker mobilisation, patient-friendly and lower costs [94]. Some time later, from the end of the 1970s onwards, several retrospective series were published [114, 204], as well as two small randomised studies in which day surgery was compared with inpatient treatment [237, 247, 262]. A recent randomised study compared how much patients valued different treatments [255]. These studies showed that day surgery is just as safe and effective, and, in addition, cheaper. In two of the three studies, patients were at least as content with day surgery $[255,262]$. In a large American cohort study, the costs of an inguinal hernia repair in a clinical setting were found to be $56 \%$ higher than those for day surgery [209]. Also in Germany, this procedure is generating less costs [317]. In addition to the few randomised studies, there are a multitude of cohort studies concerning patients successfully operated on as day surgery, under general, regional and local anaesthetics, and with both classical operation techniques as well as open tension-free repairs and endoscopic techniques. A large study conducted in Denmark noted the hospital readmission rate of $0.8 \%$ [89, 317]. Although a tension-free repair under local anaesthetic seems to be the most suitable operation, the published series showed that other surgical and anaesthesiological techniques can also be effectively used as day surgery. Only the extensive open preperitoneal approach (Stoppa technique) has not been described in the context of day surgery. 
When day surgery was in its infancy, there was a strict selection of patients with a low risk of complications (ASA I-II, age limit, length of operation $<1$ h, no serious obesity etc.). Such a strict selection is becoming less common and, in principle, an inguinal hernia repair as day surgery can be considered for every patient who has satisfactory care at home [76, 142, 245]. In this consideration, the preoperative assessment of the anaesthetist is extremely important, because he/she carries the main responsibility for the perioperative and immediately postoperative phase [245]. A number of factors will either encourage or discourage day surgery. These include hospital, physician and patientrelated factors [111]. In a hospital with considerable experience in day surgery and a good infrastructure, such as the presence of a pre-assessment consultation and a day surgery department, a large percentage of inguinal hernia treatments will take place as day surgery. The same applies to surgical factors, such as quick operations with a low percentage of complications, and anaesthesia factors, such as pain alleviation and nausea control, which make a quick discharge possible.

On a worldwide basis, there is a clear increase in the percentage of inguinal hernia repairs that are being carried out as day surgery [77, 142]. There is considerable variation between different countries, which cannot be clarified solely by the degree of acceptability of day surgery among patients and surgeons but, to a significant extent, is also determined by the healthcare financing system. In the last years (2000-2004), 35\% of inguinal hernia operations carried out in the Netherlands and 33\% in Spain were done on a day surgery basis [197, 249]; there is room for this number to be increased. In the Swedish National Registry, $75 \%$ of inguinal hernia repairs are performed in day care.

\section{Antibiotic prophylaxis}

Authors: Theo Aufenacker and Maarten Simons

Is antibiotic prophylaxis routinely indicated for elective inguinal surgery for primary inguinal hernia?

Search terms: hernia, antibiotic prophylaxis, RCT, systematic review.

\section{Conclusions}

Level In conventional hernia repair (non-mesh), 1A antibiotic prophylaxis does not significantly reduce the number of wound infections. NNT 68.

Level In open mesh repair in low-risk patients, 1B antibiotic prophylaxis does not significantly reduce the number of wound infections. NNT 80 . For deep infections, the NNT is 352 .
Level In endoscopic repair, antibiotic prophylaxis 2B does not significantly reduce the number of wound infections. NNT $\infty$.

\section{Recommendations}

Grade In clinical settings with low rates $(<5 \%)$ of A wound infection, there is no indication for the routine use of antibiotic prophylaxis in elective open groin hernia repair in low-risk patients.

Grade In endoscopic hernia repair, antibiotic B prophylaxis is probably not indicated.

Grade In the presence of risk factors for wound $\mathrm{C}$ infection based on patient (recurrence, advanced age, immunosuppressive conditions) or surgical (expected long operating times, use of drains) factors, the use of antibiotic prophylaxis should be considered.

The risk of infection following an inguinal hernia operation, with or without mesh, is between 0 and $14.4 \%$. In RCTs, the average incidence of wound infections is $4.3 \%$ in conventional repair and $2.4 \%$ in open mesh repair $[19,22,64,92,178,210,230,235,238,268,274,294$, 303, 322].

Since the use of antibiotics is not likely to increase the percentage of wound infection, the net effect of randomised controlled studies will almost always be in favour of the patients receiving prophylaxis.

In a meta-analysis on the use of antibiotic prophylaxis in 1,867 patients with non-mesh repairs, the overall infection rates were $2.88 \%$ in the prophylaxis group and $4.30 \%$ in the control group (OR 0.65, 95\% CI: 0.35-1.21) [268]. This is a non-significant reduction with a number needed to treat of 68 .

In two meta-analyses on the use of antibiotic prophylaxis in open mesh-based groin repairs, conflicting conclusions are drawn [23, 267]. In these two analyses, the same six studies are included but in one, the analysis is fixed and in the other, it was random [22, 64, 210, 230, 235, 322]. The choice of the correct method should be based on the prevalence of statistical heterogeneity (data), together with the clinical diversity and methodological diversity of the studies.

In the six studies, there is no statistical heterogeneity but clinical and methodological diversity is present and, therefore, the random method should be used. When the 
Table 7 Results of individual studies on the use of antibiotic prophylaxis in the prevention of wound infection after mesh inguinal hernia repair (RCTs)

\begin{tabular}{|c|c|c|c|c|c|c|c|c|c|c|}
\hline Reference & $n$ & \multirow[t]{2}{*}{$\begin{array}{l}\text { Mean age } \\
\text { (years) }\end{array}$} & \multirow[t]{2}{*}{$\begin{array}{l}\text { Sex, male } \\
(\%)\end{array}$} & \multirow[t]{2}{*}{ Type of antibiotic } & \multicolumn{2}{|c|}{$\begin{array}{l}\text { Infection placebo } \\
\text { group (patients, \%) }\end{array}$} & \multicolumn{2}{|c|}{$\begin{array}{l}\text { Infection } \\
\text { intervention } \\
\text { group } \\
\text { (patients, \%) }\end{array}$} & \multirow[t]{2}{*}{$P$-value } & \multirow[t]{2}{*}{ NNT } \\
\hline Endoscopic inguinal hernia mesh & repair & & & & & & & & & \\
\hline Schwetling and Bärlehner [274] & 80 & 55 & 86 & Cefuroxime $1.5 \mathrm{~g}$ & $0 / 40$ & $0 \%$ & $0 / 40$ & $0 \%$ & 1.0 & $\infty$ \\
\hline \multicolumn{11}{|c|}{ Open inguinal and femoral hernia mesh repair } \\
\hline Morales et al. [210] & 524 & 54 & 90 & Cefazolin $2 \mathrm{~g}$ & $6 / 287$ & $2.1 \%$ & $4 / 237$ & $1.7 \%$ & 0.737 & 248 \\
\hline Yerdel et al. [322] & 269 & 56 & 93 & $\begin{array}{l}\text { Ampicillin + Sulbactam } \\
1.5 \mathrm{~g}\end{array}$ & $12 / 133$ & $9.0 \%$ & $1 / 136$ & $0.7 \%$ & 0.002 & 13 \\
\hline Celdrán et al. [64] & 91 & 58 & 90 & Cefazolin $1 \mathrm{~g}$ & $4 / 49^{a}$ & $8.2 \%$ & $0 / 50^{\mathrm{a}}$ & $0.0 \%$ & 0.059 & 13 \\
\hline Oteiza et al. [230] & 247 & 57 & 85 & $\begin{array}{l}\text { Amoxicillin }+ \text { Clavulanic } \\
\text { acid } 2 \mathrm{~g}\end{array}$ & $0 / 123$ & $0.0 \%$ & $1 / 124$ & $0.8 \%$ & 0.318 & $\begin{array}{l}\mathrm{NNH} \\
124\end{array}$ \\
\hline Aufenacker et al. [23] & 1,008 & 58 & 96 & Cefuroxime $1.5 \mathrm{~g}$ & $9 / 505$ & $1.8 \%$ & $8 / 503$ & $1.6 \%$ & 0.813 & 520 \\
\hline Perez et al. [235] & 360 & 61 & 98 & Cefazolin $1 \mathrm{~g}$ & $7 / 180$ & $3.9 \%$ & $4 / 180$ & $2.2 \%$ & 0.540 & 59 \\
\hline Tzovaras et al. [303] & 379 & 63 & 94 & $\begin{array}{l}\text { Amoxicillin }+ \text { Clavulanic } \\
\text { acid } 1.2 \mathrm{~g}\end{array}$ & $9 / 189$ & $4.7 \%$ & $5 / 190$ & $2.6 \%$ & 0.4 & 48 \\
\hline Jain et al. [141] & 120 & 41 & 100 & $\begin{array}{l}\text { Amoxicillin }+ \text { Clavulanic } \\
\text { acid } 1.2 \mathrm{~g}\end{array}$ & $1 / 60$ & $1.7 \%$ & $1 / 60$ & $1.7 \%$ & 0.500 & $\infty$ \\
\hline
\end{tabular}

TAPP transabdominal preperitoneal, NNT number needed to treat

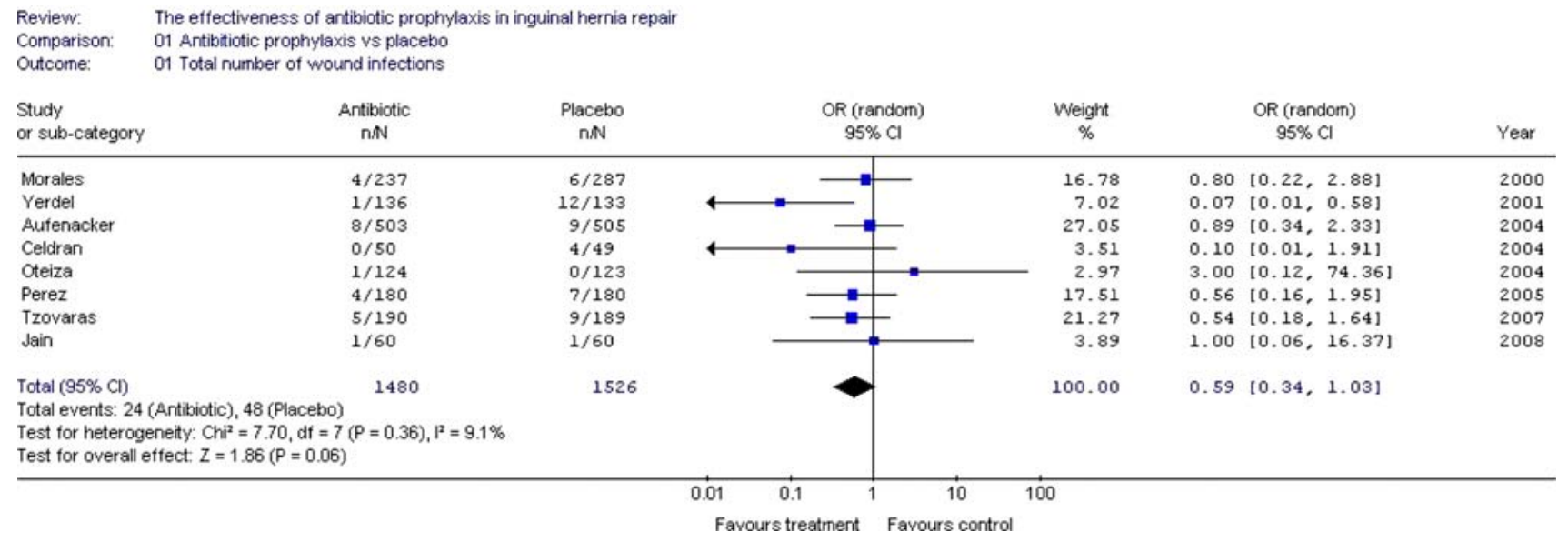

Fig. 5 Pooled data of eight studies on the use of antibiotic prophylaxis in the prevention of wound infection after mesh inguinal hernia repair

results of fixed and random analysis conflict, the choice of meta-analysis method should be preferably conservative and, in these situations, the random method should be used.

Currently, eight studies regarding open mesh repair are available; the results are displayed in Table 7 [22, 64, 141, $210,230,235,303,322]$.

In summary, one study found a significant decrease in infections. This study revealed no difference between deep infections and reported a very high percentage of superficial infections, possibly caused by long duration of operation, more usage of drains and repeated aspiration of seromas.
The results of the meta-analysis (random effect) of 3,006 patients with mesh-based groin repair are an overall infection rate of $1.6 \%$ in the prophylaxis group and $3.1 \%$ in the control group (OR 0.59, 95\% CI: 0.34-1.03). This is not a significant reduction, with a number needed to treat of 80 (Fig. 5).

For the prevention of a deep infection, data is available on 2,103 patients. The deep infection rate is $0.3 \%$ in the prophylaxis group and $0.6 \%$ in the placebo group (OR $0.50,95 \%$ CI: $0.12-2.09$ ). The reduction is not significant and the number needed to treat is 352 to prevent one deep infection. 


\section{Training}

Authors: Marc Miserez and Maarten Simons

What is the learning curve and training in inguinal hernia repair?

Search terms: hernia, training, learning curve.

\section{Conclusions}

Level The learning curve for performing endoscopic 2C inguinal hernia repair (especially TEP) is longer than that for open Lichtenstein repair, and ranges between 50 and 100 procedures, with the first $30-50$ being the most critical.

For endoscopic techniques, adequate patient selection and training might minimise the risks for infrequent but serious complications in the learning curve.

There does not seem to be a negative effect on outcome when operated by a resident versus an attending surgeon.

Specialist centres seem to perform better than general surgical units, especially for endoscopic repairs.

\section{Recommendations}

Grade (Endoscopic) hernia training with adequate C mentoring should be started with junior residents.

Grade All surgeons graduating as general surgeons D should have a profound knowledge of the anterior and posterior preperitoneal anatomy of the inguinal region.

Complex inguinal hernia surgery (multiple recurrences, chronic pain, mesh infection) should be performed by a hernia specialist.

Surgeons recognise technical issues, experiences in deciding to act and manual skills as major predictors of outcome [285]. A learning curve for a specific procedure can be evaluated by means of operative times, but mainly by the rate of conversions (for endoscopic surgery) and complications. It is generally believed that the learning curve for performing endoscopic inguinal hernia repair is longer than for open Lichtenstein repair, although the Lichtenstein technique also has a learning curve with respect to the prevention of recurrence and the prevention of chronic groin pain. However, this learning curve seems to be more favourable than that for the endoscopic techniques [298]. This is especially the case for TEP, due to a limited working space and different appreciation of the usual anatomical landmarks seen from inside the peritoneal cavity or through an anterior approach. The risk of serious complications may be higher during the learning curve period, and adequate patient selection and training might minimise the risks for rare but serious complications.

Very limited data are available on the learning curves for endoscopic repair, but it is suggested that operators become experienced after between 50 and 100 procedures, with the first $30-50$ being the most critical [40, 79, 86, 95, 174, 184, 312, 313]. Lamb et al. showed for TEP that recurrence rates (median follow-up time 7 years) are $<2 \%$ if more than 80 procedures had been performed and, recently, the learning curve specifically for TAPP was suggested to be $>75$ [192]. Of course, this number is clearly dependent on the structure of the training (programme), such as the type of supervision and the expertise of the trainer [184]. The number of 250, especially in surgeons aged 45 years and above as reported in the VA trial, has been criticised because of several reasons (learning curve effect, size of the mesh) [207, 215, 216].

There is a substantial variation in the amount of training and supervision given for inguinal hernia repair and, currently, an increased number of complications during the learning curve period due to nonoptimal training conditions is no longer acceptable [75]. Although there are no RCTs concerning hernia surgery that compare the outcome of trainee versus surgeon or specialist, most surgical data, even on pancreatic surgery, show no negative effect on outcome when operated by a resident versus an attending surgeon [69, 139, 246, 276]. Of course, the quality and extent of supervision is an important factor in outcome [93]. Registration in a prospective database in Scotland showed that junior residents, when supervised by a senior resident or a consultant surgeon (for open surgery) and senior residents, whether supervised or unsupervised (for open and endoscopic surgery), obtained comparable recurrence rates as the consultant surgeons [261]. More or less comparable data on morbidity and recurrence rate (although associated with a higher operation time for residents) were found for the Lichtenstein procedure [74]. In a retrospective analysis of 264 TEP interventions, mainly performed by surgical residents under the guidance of a single staff surgeon, the mean operation time was $85 \mathrm{~min}$ (unilateral hernias) and the recurrence rate was $2 \%$, with a mean follow-up of 3.5 years [120]. In a TAPP inguinal hernia repair, the learning curve seemed to be shorter for junior trainees than for the senior surgeons (who were training them now) years before [40]. Other conflicting data state that surgical training for endoscopic treatment of inguinal hernias is associated with a longer 
operation time and hospital stay, and with higher morbidity and costs [29].

For the Lichtenstein repair, a post-hoc analysis of the VA trial data (where surgical residents assisted during the whole operation by an attending surgeon who performed most of the repairs) showed higher recurrence rates (but not other complications) for junior residents operating versus senior residents. For the endoscopic repair, the attending effect was so important that no resident effects on the recurrence and complication rates could be detected [319]. (Endoscopic) hernia training with adequate mentoring should, therefore, probably be started with junior residents.

Specialist centres seem to perform better than general surgical units, especially for endoscopic repairs and complex inguinal hernia surgery (multiple recurrences, chronic pain, mesh infection etc.) should, thus, be best performed by a hernia specialist $[40,225,291]$. It is unclear whether subspeciality training, centre volume and/or surgeon volume are equally important to determine the outcome [125], but for many procedures, the observed associations between hospital volume and operative mortality are largely mediated by surgeon volume [39]. For open paediatric inguinal hernia repair (excluding premature babies), hernia recurrence was higher in the general surgery group compared with paediatric surgeons; in addition, only among paediatrics surgeons-having a higher surgical volumewas the estimated risk of hernia recurrence independent of surgical volume [43]. On the other hand, the results of nonexpert surgeons and even supervised residents using the Lichtenstein repair for primary inguinal hernias showed comparable excellent results to those of the experts [74, 277].

All surgeons graduating as general surgeons should have a profound knowledge of the anterior and posterior preperitoneal anatomy of the inguinal region. As long as they also treat recurrent hernias, it is logical to be adequately trained in both the anterior and posterior approach to the groin by means of adequately tested (pre)clinical training models and curricula [126]. Most authors agree that the two major techniques to be taught early during surgical residency are the Lichtenstein technique for the anterior approach and the endoscopic techniques for the posterior approach. All new procedures should be compared to these techniques. In order to decrease patient exposure to learning curve errors during inguinal hernia repair and due to the current lack of realistic hands-on simulation models, at least $30-50$ of each procedure should be performed during residency, with adequate mentoring by a motivated expert surgeon (and instructor!), provided the resident already has experience with endoscopic cholecystectomy ( endoscopic experience) [79, 86, 184, 312].

Every surgical resident graduating in the USA in 1999 performed during the whole residency period seven endoscopic/endoscopic and 50 open procedures, on average [79]. Currently, a resident should ideally have performed at least ten complete endoscopic/endoscopic and 50 open inguinal hernia repairs independently [254]. In addition, a specific effort for postgraduate training in endoscopic inguinal hernia repair should be made for inexperienced consultant surgeons.

\section{Anaesthesia}

Authors: Par Nordin and Sam Smedberg

Can an open inguinal hernia operation under local anaesthesia be performed with the same patient satisfaction? Is this safer and more cost-effective than other anaesthesia techniques? Should regional anaesthesia be avoided?

Search terms: herniorrhaphy, groin hernia, local anaesthesia.

\section{Conclusions}

Level Open anterior inguinal hernia techniques can be 1B satisfactorily performed under local anaesthetic. Regional anaesthesia, especially when using high-dose and/or long-acting agents, has no documented benefits in open inguinal hernia repair and increases the risk of urinary retention.

\section{Recommendations}

Grade It is recommended that, in the case of an open A repair, local anaesthetic is considered for all adult patients with a primary reducible unilateral inguinal hernia.

Grade Use of spinal anaesthesia, especially using highB dose and/or long-acting anaesthetic agents, should be avoided.

General anaesthesia with short-acting agents and combined with local infiltration anaesthesia may be a valid alternative to local anaesthesia.

Ideally, inguinal hernia repair should be performed using a simple and safe anaesthetic technique that is acceptable for the patient and easily mastered in general surgical practice. The technique should carry a low morbidity risk and also be cost-effective. Postoperative side effects and prolonged hospital stay after groin hernia surgery are often related to the effects of anaesthesia.

General anaesthesia can provide the surgeon with optimal operating conditions in terms of patient immobility and muscular relaxation. Modern general anaesthesia with short-acting agents and combined with local infiltration 
anaesthesia is safe and fully compatible with day-case surgery [282]. Disadvantages are risk for airway complications, cardiovascular instability, nausea and vomiting. Furthermore, urinary complications and recovery from central hypnotic effects may prolong the hospital stay.

Regional anaesthesia for groin hernia repair can be provided by either subarachnoid (spinal), epidural techniques or, more uncommonly, paravertebral techniques [156]. Spinal anaesthesia regularly results in urine retention, which results in prolonged postoperative recovery [98, 221, 264, 290].

In recent years, improvements of the regional anaesthetic techniques have been made with the use of more short-acting local anaesthetic agents. Also, the use of additional spinal opioids combined with a reduction in the amount of spinal doses may reduce the postoperative side effects.

The open treatment of primary reducible inguinal hernias in adults is nearly always possible under local anaesthesia $[59,149,150]$ and can be provided by a local infiltration technique $[16,17]$ or by a specific blockade of the ilioinguinal and iliohypogastric nerves or a combination of the two methods (see Appendix 4) [80]. The administration is technically quite easy but it requires training and is only successful if the surgeon handles the tissues gently, has patience and is fully conversant with the technique $[80,241]$. Intraoperative pain seems to be the most common reason for dissatisfaction with local anaesthesia [222, 295]. Some patients may prove to be unsuitable for local anaesthesia, notably very young patients, anxious patients, the morbidly obese and patients with suspected incarceration or strangulation. Whether scrotal hernias and obese patients are suitable depends entirely upon the surgeon's familiarity with the technique [80, 241].

Fourteen randomised studies comparing local anaesthesia with general and/or regional anaesthesia $[11,37$, $104,112,113,118,158,221,227,231,273,282,295,310]$ and one comparing general with regional anaesthesia [52] was found. One study did not reveal any difference [227], while the others bear witness to the advantages for local anaesthetic, such as less postoperative pain, less anaesthesia-related complaints, less micturition difficulties, faster discharge and faster short-term recovery.

Cost comparisons for the anaesthetic alternatives have given similar results [31, 57, 151, 223, 282]. Local anaesthesia provides cost advantages over both regional and general anaesthesia, regarding both total intraoperative as well as postoperative costs. Of three RCTs [223, $227,282]$, two found local to be cheaper than both general and spinal anaesthesia [223, 282], while one observed no major difference between local and general anaesthesia [227].
Local anaesthesia carries a lower mortality risk in both elective and emergency operations [219].

\section{Postoperative recovery}

Authors: Rene Fortelny and Maciej Smietanski

Which technique gives the fastest postoperative recovery?

Search terms: inguinal hernia, treatment, recovery, pain, outcome.

\section{Conclusions}

Level Endoscopic inguinal hernia techniques result in 1A an earlier return to normal activities or work than the Lichtenstein technique.

\section{Recommendations}

Grade It is recommended that an endoscopic technique A is considered if a quick postoperative recovery is particularly important.

Postoperative recovery is defined as a return to normal activities of daily living and the resumption of paid work.

The most important economic short-term effect after inguinal hernia surgery is the recommended postoperative recovery time, which was formerly 6 weeks on average $[266,284]$. This figure originates from the period in which almost every surgeon treated inguinal hernia by means of an anterior approach without using mesh [25]. The duration of convalescence varies considerably, basically due to the variation in recommendations and on the level of the patients' preoperative activity [56]. The resumption of work relies on different factors and is not only dependant on the operation technique $[44,255,258]$. The main cause of prolonged recovery is predominantly pain [56]. In addition, co-morbidity and cultural background affect the time of recovery [197]. An early resumption of daily activities and work has been advocated in all published recommendations but has not generally been adopted [91]. Based on these widely accepted facts, the patients should be informed that they can immediately return to all of their usual activities of daily life if pain permits $[56,91]$.

All tension-free hernia operation techniques (open, e.g. Lichtenstein procedure and plug and patch repair, or endoscopic approach, e.g. TAPP and TEP) have been analysed in various trials and result in a quicker postoperative recovery $[41,46,56,68,129,147,168,176,178$, 197, 198, 272, 311].

The meta-analyses revealed that, after an open mesh procedure, patients recovered four days earlier on average than after a conventional repair, and recovered seven days earlier on average following an endoscopic operation than 
after an open technique with mesh $[41,46,68,115,129$, 168, 176, 197, 198, 272, 275].

The recovery was mostly measured using (non)-validated questionnaires or by enquiring about an end point (only the resumption of normal activities and/or paid work): so-called ADL questionnaires or a generic quality of life questionnaire (SF-36 or EuroQol) [185, 187, 296, $316]$.

The results of these quality of life studies demonstrate that the recovery of hernia patients is not only restricted to the dimension of pain. It seems obvious that favourable physical outcomes facilitate the return to normal social life and productivity [198]. Although the social and psychological impairment has already been observed in hernia patients, these aspects of recovery have, so far, been neglected by the surgical research in the field.

However, the growing body of literature underlines the importance of quality of life assessment in future studies on hernia repair.

In a number of studies, this quicker postoperative recovery was objectively confirmed by abdominal muscle exercises being carried out [82, 187, 233].

\section{Aftercare}

\section{Author: Maarten Simons}

Is a lifting, sports or work ban indicated following inguinal hernia surgery?

Search terms: groin hernia, herniorrhaphy, aftercare, postoperative regime, postoperative recommendations, guidelines.

\section{Conclusions}

Level The imposition of a temporary ban on lifting, 3 participating in sports or working after inguinal hernia surgery, is not necessary. Probably a limitation on heavy weight lifting for 2-3 weeks is enough.

\section{Recommendations}

Grade It is recommended that limitations are not placed $\mathrm{C}$ on patients following an inguinal hernia operation and patients are, therefore, free to resume activities. "Do what you feel you can do." Probably a limitation on heavy weight lifting for 2-3 weeks is enough.

In a single prospective trial, two postoperative regimes (after Bassini technique) were compared with each other using a recurrence within 1 year as an end point [292]. The resumption of heavy work after 3 weeks was compared with that after about 10 weeks and has no influence on the recurrence rate.

If (after Lichtenstein technique under local anaesthetic) postoperatively patients were allowed to do what they could, and only excessive sport and heavy work were limited during the first 3 weeks, it resulted in $>75 \%$ doing their own shopping without assistance within 6 days. Following light work, patients resumed work after an average of 6 days, and resumed heavy work 4 days after the proposed resting period was over [58].

Recommendations about driving after an inguinal hernia operation differ considerably. A study showed that, after seven days, the normal response time was achieved in $82 \%$ of cases following an endoscopic repair, in $64 \%$ of cases after Lichtenstein operation and in $33 \%$ of cases after Bassini technique [320]. In the Lichtenstein clinic, the opinion is that driving can be resumed straight away [13]. It is hardly surprising that every surgeon gives a different recommendation [140].

\section{Postoperative pain control}

Authors: Par Nordin and Sam Smedberg

What is the best method for realising an effective postoperative pain control?

Search terms: herniorrhaphy, local anaesthetic(s), groin hernia, local anaesthesia, local infiltration.

\section{Conclusions}

Level Wound infiltration with a local anaesthetic 1B results in less postoperative pain following inguinal hernia surgery.

\section{Recommendations}

Grade Local infiltration of the wound after hernia repair A provides extra pain control and limits the use of analgesics.

In addition to the medicinal postoperative analgesia, which is not discussed further, there are sufficient studies which demonstrate that wound infiltration with a local anaesthetic results in less postoperative pain than the administration of placebos [81, 302].

http://www.postoppain.org covers evidence-based recommendations.

\section{Complications}

Authors: Sam Smedberg and Par Nordin

How frequent are complications after inguinal hernia operations, and can the risk of complications be reduced? 
Which are the specific complications following inguinal hernia operation and how should they be treated?

Search terms: inguinal hernia, clinical trial, randomised controlled trial and the terms associated with the complication.

The overall risk of complications after inguinal hernia operations reported vary from 15 to $28 \%$ in systematic reviews [41, 272]. With active monitoring such as phone calls, questionnaires or clinical examination, the rates have been reported to be higher, ranging from 17 to $50 \%$ [87, 215, 222]. The most frequent early complications were haematomas and seromas $(8-22 \%)$, urinary retention and early pain, and late complications were mainly persistent pain and recurrences [41, 197, 272]. Lifethreatening complications were rarely reported [197]. Risks of complications are related to several factors, as described below. Hernia surgery is reconstructive surgery and it appears that meticulous technique pays off, e.g. regarding nerve damage and recurrences, irrespective of what method of repair has been used (level 2). Differences in the results between methods will be described in more detail.

Which are the specific complications following inguinal hernia operation and how should they be treated?

Search terms: inguinal hernia, clinical trial, randomised controlled trial and the terms associated with the complication.

In this chapter, the literature on chronic pain and related conditions will be evaluated. For other complications, only recommendations will be stated due to the low level of evidence and for the sake of readability.

Inguinal hernia surgery has a relatively low risk of perioperative and early postoperative complications of some significance. A study of the literature, however, reveals a number of issues:

- Results published by specialist centres (level 3-4) are much better than the results from everyday practice (level 1-3)

- Open inguinal hernia surgery and endoscopic inguinal hernia surgery have specific technique-related complications

- The definitions of complications vary between reports, which makes the evaluation of results difficult

- Postoperative chronic pain is more frequent than previously understood, and has recently become one of the most important primary endpoints in hernia surgery

- Serious peri- and postoperative complications in respect of visceral and vascular injuries are rare
- The risk for serious complications appears to be lower for open repair compared with endoscopic techniques [198]

\section{Haematoma}

Serious, transfusion-requiring haemorrhages rarely occur in the case of open and endoscopic inguinal hernia surgery. The incidence of inguinal haematomas is lower for the endoscopic techniques than with open repair.

In the case of open surgery, the risk of haematomas varies between 5.6 and 16\%. When endoscopic techniques were used, the risk varies between 4.2 and $13.1 \%$ [41, 197, 271]. A small haematoma can be treated conservatively. For larger haematomas, which also give rise to a lot of pain and/or tension on the skin, an evacuation of the haematoma under anaesthetic should be considered.

Results of the systematic review Haematoma after open mesh versus open non-mesh in 13 trials: 82/1,479 $(5.5 \%)$ versus 104/1,593 (6.5\%); OR 0.93 (0.68-1.26) n.s. [116].

Haematoma after endoscopic technique versus open technique in 33 trials: $238 / 2,747(8.6 \%)$ versus $317 / 3,007$ (10.5\%); OR 0.72 (0.60-0.87) $P=0.0006$ [197].

\section{Seroma}

The risk of seroma formation varies between 0.5 and $12.2 \%$. These incidences are significantly higher for the endoscopic techniques than for open repairs [41, 197, 271]. Most seromas disappear spontaneously within a period of 6-8 weeks. Should a seroma persist, it can be aspirated. Infection following the aspiration of seromas is regularly described. Studies concerning postoperative drainage to prevent seromas are contradictory. In two RCTs of patients following open intervention, no advantage was observed in a series of 100 patients, whereas in another series of 301 patients, clear advantages were revealed for a drainage period of $24 \mathrm{~h}$ [34, 234]. The risk of seroma is rarely big enough to necessitate leaving a drain, except in the case of excessive diffuse blood loss or patients with (iatrogenic) coagulopathies.

Results of the systematic review Seroma after open mesh versus open non-mesh in 13 trials: 38/1,548 (2.4\%) versus 24/1,497 (1.6\%); OR 1.52 (0.92-2.52) n.s. [116].

Seroma after endoscopic technique versus open technique in 28 trials: 139/2,408 (5.7\%) versus 101/2,679 (3.7\%); OR 1.58, 95\% CI: (1.20-2.08) $P=0.001$ [197]. 


\section{Recommendations}

Grade It is recommended in the case of open surgery to B operatively evacuate a haematoma which results in tension on the skin.

It is recommended that wound drains are only used where indicated (much blood loss, coagulopathies).

Grade It is recommended that seromas are not C aspirated.

\section{Wound infection}

Open and endoscopic surgery. The risk of a wound infection following an inguinal hernia operation with or without mesh should be below 5\%. The use of mesh in inguinal hernia repair is not associated with a higher risk of wound infection. Superficial infections are rare after endoscopic techniques. The risk is probably about $1-3 \%$ for open surgery and less than $1 \%$ after endoscopic surgery [23, 41, 197, 268, 271, 272, 293].

Results of the systematic review (mainly superficial wound infections) Wound infection in the case of open mesh versus open non-mesh techniques for inguinal hernias in 16 trials: 59/1,702 (3.4\%) versus 52/1,814 (2.8\%); OR 1.24 $(0.84-1.84)$ n.s. [116].

Wound infection in the case of endoscopic versus open techniques in 29 trials: $39 / 2,616(1.5 \%)$ versus $92 / 2,949$ (3.1\%); OR $0.45(0.32-0.65) P<0.0001$ [118].

Deep infections are rare and do not have to lead to the removal of the mesh when monofilament materials are used [293]. Drainage and antibiotics are usually sufficient. However, removal of the mesh has been described; this is virtually inevitable in the presence of a multifilament mesh.

\section{Urinary retention and bladder damage}

The urinary retention incidence varies with a multiplicity of operative and peri-operative factors. In a review of the literature in the period 1966-2001 on urinary retention in relation to anaesthetic technique, 70 non-randomised and two randomised studies were found [144]. The incidence of urinary retention with local anaesthesia was $0.37 \%$ (33 in 8,991 patients), with regional anaesthesia being $2.42 \%$ (150 in 6,191 patients) and with general anaesthesia being $3.00 \%$ (344 in 11,471 patients). The inhibitory effect of regional and general anaesthesia on bladder function would explain the results. In two metaanalyses of RCTs comparing endoscopic and open mesh or open non-mesh techniques, respectively, no significant differences in postoperative urinary retention were found [41, 272]. Preperitoneal placement of mesh with the TEP technique was found not to cause urinary retention by outflow obstruction or alteration of the bladder contractility [175]. The volume of intravenous postoperative fluid administered is a significant risk factor [164].

Bladder damage can occur after both endoscopic and open surgery. It is an uncommon complication that is somewhat more frequent in transabdominal endoscopic operations. In the endoscopic literature, it varies from $4.2 \%$ in smaller series to $0.2 \%(8 / 3,868), 0.1 \%(1 / 686)$ and $0.06 \%(1 / 3,229)[2,3,236,242,291]$.

Predisposing factors are a full bladder, exposure of the retropubic space (particularly after prostate interventions, irradiation or TAPP) and the opening of the transversalis fascia/peritoneum in direct hernias (level 3).

\section{Recommendations}

Grade It is recommended that the patient empties his/ D her bladder prior to endoscopic and open operations.

It is recommended that the fascia transversalis/ peritoneum is opened with restrictivity in open surgery of direct hernias. Take care that the bladder might be herniated.

\section{Ischaemic orchitis, testicular atrophy and damage to the ductus deferens}

Testicular complications occur after both open and endoscopic hernia surgery. No significant difference in incidence between open and endoscopic techniques were found in two meta-analyses of RCTs of high quality, the total number of cases being 51/7,622 (0.7\%) [41, 272].

Postoperative ischaemic orchitis usually develops within 24-72 h after the operation. It may result in testicular necrosis within days or have a slower course, resulting in testicular atrophy over a period of several months. Acute ischaemia can be prevented by leaving the cremasteric vessels intact [257]. There is an increased risk of ischaemic orchitis after recurrent open hernia surgery and after dissection below the level of the pubic tubercle, e.g. after complete excision of scrotal hernias [315]. Minimising cord dissection is recommended. Extensive dissection of the pampiniform plexus or tight closure of the internal ring may result in damage to the testicular vessels and/or ductus deferens [173]. Transection of the hernia sac leaving the distal part in situ is recommended to reduce the risk of ischaemic orchitis. Thrombosis of testicular veins following extensive dissection is considered to be the cause of ischaemic orchitis [315]. 


\section{Recommendations}

Grade It is recommended that, in the case of large D hernia sacs, transection of the hernia sac is performed and the distal hernia sac is left undisturbed, so as to prevent ischaemic orchitis. Damage to the spermatic cord structures should be avoided.

\section{Damage to the intestines}

Damage to the intestines rarely occurs in open hernia surgery and is, in general related, to an intervention for incarcerated hernia. In endoscopic hernia surgery, the risk is low; however, it occurs more frequently, from 0.0 to $0.21 \%$ [96, 99, 272, 291]. Risk factors are previous major lower (open) abdominal interventions, previous radiotherapy of pelvic organs and insufficient insulation of endoscopic instruments during coagulation.

\section{Recommendations}

Grade It is recommended that patients with previous D major lower (open) abdominal intervention or previous radiotherapy of pelvic organs do not undergo endoscopic inguinal hernia surgery.

\section{Bowel obstruction}

The incidence of intestinal obstruction after TAPP inguinal hernia operation varies from 0.07 to $0.4 \%$ [82, 99, 236, 301]. It may also develop after TEP operation; however, this occurs less frequently [291].

Bowel obstruction can develop due to adhesions between the mesh and the intestines [193, 194], e.g. by inadequate closure of a peritoneal lesion [291]. Rare cases of bowel obstruction in port-site hernias have also been described, especially after TAPP.

\section{Recommendations}

Grade It is recommended that, due to the risk of D intestinal adhesion and the risk of bowel obstruction, the extraperitoneal approach (TEP) is used for endoscopic inguinal hernia operations. It is recommended that trocar openings of $10 \mathrm{~mm}$ or larger are closed.

\section{Vessel damage}

Damage to the large vessels rarely occur in the case of an open inguinal hernia operation, and is mostly described in the McVay technique [27]. Damage to the epigastric vessels may occur more frequently, the significance of which, however, is obscure, since dividing the epigastric vessels is part of the original method in open preperitoneal techniques such as the Stoppa operation and its unilateral variety [286, 314].

In TAPP, blind introduction of the Veress needle and trocars may damage the aorta, vena cava and iliac vessels. The incidence is low and only occasional cases are reported in the hernia literature. In a large series, an incidence of $0.06-0.13 \%$ is reported $[199,236]$. Damage to the inferior epigastric vessels as a consequence of trocar introduction has an incidence of $0-0.07 \%$ [96, 159161].

\section{Recommendations}

Grade It is recommended that the first trocar at D endoscopic hernia surgery (TAPP) is introduced by the open technique.

\section{Mesh rejection and migration}

Mesh migration is described after all varieties of mesh repairs but for plug techniques in particular [180]. Migration to the intestines, urinary bladder, femoral vein, preperitoneal space and the scrotum have been reported $[8$, $66,67,128,180,229]$. Mesh rejection following different surgical techniques and mesh materials have also been reported [26, 102, 135, 213, 265, 269]. In a review of the literature on mesh plug migration, it was concluded that plug migration after open inguinal hernia surgery can be avoided if proper attention to detail is used at the time of initial repair [143].

\section{Specific endoscopic complications}

Pneumatic complications. Pneumomediastinum, pneumothorax and subcutaneous emphysema (pneumoscrotum) are rarely reported and are mostly related to a high insufflation pressure [51, 97, 253]. Subcutaneous $\mathrm{CO}_{2}$ emphysema can occur due to the incorrect placing of the Veress needle or leakage of $\mathrm{CO}_{2}$ along the trocars [232].

Carbon dioxide insufflation-related complications. $\mathrm{CO}_{2}$ insufflation can result in hypercapnia, acidosis and hemodynamic changes [65]. Hypercapnia was reported in $2 / 686$ patients [99].

Trocar complications. Trocar hernias vary from 0.06 to $0.4 \%$ for the TAPP to $0.7 \%$ for various endoscopic interventions $[96,99,236]$. 


\section{Chronic pain, nerve damage and neuralgia}

Definition: by the International Association for the Study of Pain (IASP), chronic pain is defined as pain lasting for 3 months or more [1].

What causes chronic pain after inguinal hernia surgery, can it be prevented and how can it be treated?

\section{Conclusions; causes and risk factors}

Level The risk of chronic pain after hernia repair with 1B mesh is less than after non-mesh repair. The risk of chronic pain after endoscopic hernia repair is lower than after open hernia repair.

Level The overall incidence of moderate to severe 2A chronic pain after hernia surgery is around 10$12 \%$.

The risk of chronic pain after hernia surgery decreases with age.

Level Preoperative pain may increase the risk of 2B developing chronic pain after hernia surgery. Preoperative chronic pain conditions correlate with the development of chronic pain after hernia surgery.

Severe early postoperative pain after hernia surgery is correlated to the development of chronic pain.

Females have an increased risk of developing chronic pain after hernia surgery.

\section{Conclusions; prevention of chronic pain}

Level Material-reduced meshes have some advantages

1B with respect to long-term discomfort and foreign-body sensation in open hernia repair (when only considering chronic pain).

Level Prophylactic resection of the ilioinguinal nerve 2A does not reduce the risk of chronic pain after hernia surgery.

Level Identification of all inguinal nerves during open 2B hernia surgery may reduce the risk of nerve damage and postoperative chronic groin pain.

\section{Conclusions; treatment of chronic pain}

Level A multidisciplinary approach at a pain clinic is an

3 option for the treatment of chronic postherniorrhaphy pain.

Surgical treatment of specific causes of chronic post-herniorrhaphy pain can be beneficial for the patient, such as the resection of entrapped nerves, mesh removal in mesh-related pain, removal of endoscopic staples or fixating sutures.

Grade The use of lightweight/material-reduced/largeA pore $(>1000-\mu \mathrm{m})$ meshes inopen inguinal hernia repair can be considered to decrease longtermdiscomfort (when only considering chronic pain).

Endoscopic surgery is superior to open mesh (when only consideringchronic pain), if a dedicated team is available.

Grade It is recommended that risks of the development B of chronic postoperative pain are taken into account when the method of hernia repair is decided upon.

It is recommended that inguinal nerves at risk (three nerves) are identified at open hernia surgery.

Grade It is recommended that a multidisciplinary C approach is considered for the treatment of chronic pain after hernia repair.

It is recommended that surgical treatment of chronic post-herniorrhaphy pain as a routine is restricted in the lack of scientific studies evaluating the outcome of different treatment modalities.

Chronic pain after hernia operation, causes and risk factors

In a systematic review of the literature in the period 19872000 , the frequency of chronic pain after hernia repair, reported in 40 studies, ranged from 0 to $53 \%$. In six studies where pain was the primary outcome of interest, the frequency was highest, in the range 15-53\% [242]. This observation was confirmed in a systematic review of the 
pain literature from 2000 through April 2004 [2]. Overall, moderate to severe pain was experienced by $10-12 \%$ of the patients $[2,242]$.

Intraoperative nerve damage in relation to the development of chronic pain has been discussed [2]. The risk of nerve damage is reduced at endoscopic surgery. The incidence of chronic pain is reported to be lower after TAPP and TEP compared to open surgery, with or without mesh $[2,3,72,165,215,272]$. Other manifestations of nerve lesions like numbness and paresthesia are also fewer following endoscopic surgery [41, 272]. A meta-analysis of 41 trials of endoscopic versus open groin hernia repair with 7,161 participants (individual patient data available for 4,165 cases) revealed less persisting pain and numbness after endoscopic repair [115].

Patients undergoing reoperative surgery for recurrent hernia were at risk of developing chronic neuralgia with a four-fold higher rate of moderate or severe chronic pain $[2$, 242].

Most studies comparing mesh with non-mesh repair report less chronic pain with mesh repair [2, 72, 242]. The EU Hernia Trialists Collaboration review concluded that significantly less pain followed mesh repairs in randomised studies of open flat mesh versus non-mesh, TAPP versus non-mesh and TEP versus non-mesh [72].

In an RCT comparing three open mesh techniques, long-term follow-up with a postal questionnaire including a VAS pain score was completed for 319 (95.8\%) patients. Chronic pain was found to be related to younger age [217], which has also documented by others [2], and related to stronger pain directly after the operation [2, 217]. At 5-year follow-up of 867 patients (81.2\%) in an RCT comparing TAPP and Shouldice inguinal hernia repair, no differences in late discomfort were found. However, severe pain during the first postoperative week was a risk factor for late discomfort in the Shouldice group (OR 2.25, $P=0.022$ ) but not in the TAPP group [38].

Preoperative pain may increase the risk of developing chronic pain according to some studies, and preoperative chronic pain conditions such as headache, back pain and irritable bowel syndrome have been found to be significantly correlated with the development of chronic pain [2].

Females have been found to have an increased risk of developing postoperative chronic pain [2].

\section{Prevention of chronic pain}

Attempting to reduce the risk of early postoperative pain and late chronic groin pain, the operative handling of inguinal nerves have been studied, methods of mesh fixation have been compared and meshes inducing less inflammatory reaction developed.
In a systematic review on nerve management during open hernia repair, three randomised studies were found reporting that chronic pain after identification and division of the ilioinguinal nerve was similar to that after identification and preservation of the nerve [318].

Two cohort studies suggested that the incidence of chronic pain was significantly lower after the identification of all inguinal nerves compared to without the identification of any nerves [318].

Fibrin glue and non-fixation techniques have been compared to mesh fixation with staples and tackers in endoscopic hernia operations. Reduced early postoperative pain with the non-stapling techniques were found, but there were no differences in the risks of late chronic pain in two studies [173, 281]. In one study, the risk of chronic pain at 1 year was lower with fibrin glue [173].

Lightweight mesh versus standard polypropylene mesh was studied in 590 patients operated with the Lichtenstein technique. At 3-year follow-up, there were no differences in neuralgic pain, hypoaesthesia or hyperaesthesia between the groups. There were no major differences in response to the pain questionnaire, except that fewer men with lightweight mesh had pain when rising from lying down to a sitting position. Significantly more men in the standard mesh group could feel the mesh in the groin, 22.6 versus $14.7 \% ; P=0.025\left(\chi^{2}\right.$ test $)[50]$.

Earlier randomised studies of 117 and 321 patients, respectively, indicated that the use of lightweight mesh was associated with significantly less pain during exercise after 6 months and less pain of any severity at 12 months in the lightweight group [228, 244].

\section{Treatment of chronic pain}

There are no randomised studies on the treatment of chronic pain after hernia surgery. All studies analysed in a systematic review of the surgical management of chronic groin pain after inguinal hernia repair were found to suffer from poor methodological quality in different aspects [3].

The recommendation that patients suffering from severe groin pain more than 3 months postoperatively should be referred to a pain clinic was based on observations in a nationwide follow-up study [73]. Patients with severe groin pain often had a history of a pain syndrome, and $71 \%$ of patients with severe groin pain 3 months postoperatively still had pain after 2.5 years [73].

Step by step diagnosis and treatment of chronic postoperative groin pain in a multidisciplinary treatment centre resulted in 16 cures and 22 improvements among 47 studied cases. Surgery was performed in selected cases not described in detail [109].

Resection of one or more inguinal nerves has been successful. Eighty percent of pain-free patients at 1 month 
in a series of 225 patients was reported [14]. Long-term follow-up and evaluation of neurectomy is, however, sparse.

In a series of 117 re-explorations because of pain after hernia surgery, 20 had a previous mesh repair. All 20 meshes were removed, 16 including neurectomy. There was a $60 \%$ success rate [132].

\section{Sexual complaints}

Ejaculatory pain and sexual dysfunction related to inguinal hernia are evaluated in only a few studies, and prophylactic measures or treatments have, till now, not been suggested. Preoperative hernia-related sexual dysfunction in 11 patients (15\% of a study group of 73 ) was successfully treated by the hernia operation in all cases [323]. In the same study group, postoperative sexual dysfunction appeared in ten patients and recovered spontaneously in six within 12 months.

In a Danish nationwide questionnaire study of pain related sexual dysfunction, executed in September 2004, all men aged 18-40 years undergoing inguinal herniorrhaphy, mainly open mesh repairs, between October 2002 and June 2003 ( $n=1,015$ ) were included, with a response rate of $63.4 \%$. Genital or ejaculatory pain was found in $12.3 \%$ and $2.8 \%$ reported a moderate to severe impairment of sexual activity [4]. These symptoms were assessed more in detail in ten patients and compared with 20 patients with postoperative chronic pain without sexual dysfunction [5]. The pain was specifically located at the external inguinal ring in ejaculatory pain patients, and psychosexual interview concluded that the pain was of somatic origin. The symptoms were related to deterioration in the overall quality of life and sexual function of the patients.

\section{Mortality}

Mortality risk following elective inguinal hernia repair is low, even at older age. It is, in all series, less than $1 \%$ and in a Swedish register study not raised above that of the background population [219]. In a Danish study among 26,304 patients, this was $0.02 \%$ under the age of 60 years and $0.48 \%$ above 60 years of age [32].

An emergency operation carries a substantial mortality risk [12, 32, 169, 170, 196, 219, 248]. In the Danish study, the mortality was $7 \%$, and in the Swedish database, it was increased seven-fold after emergency operations and 20fold if bowel resection was undertaken [32, 219].

Women have a higher mortality risk than men due to a greater risk for emergency procedure irrespective of hernia anatomy and a greater proportion of femoral hernia. After femoral hernia operation, the mortality risk was increased seven-fold for both men and women [219].

\section{Recommendations}

Grade It is recommended to offer patients with femoral B hernia early planned surgery, even if symptoms are vague or absent.

Grade It is recommended to intensify efforts to improve D the early diagnosis and treatment of patients with incarcerated and/or strangulated hernia.

\section{Costs}

Authors: Timo Heikkinen and Marc Miserez

What is the most cost-effective operation for the treatment of primary inguinal hernia?

Search terms: inguinal hernia, costs.

\section{Conclusions}

Level From the perspective of the hospital, an open mesh 1B procedure is the most cost-effective operation in primary unilateral hernias. From a socioeconomic perspective, an endoscopic procedure is probably the most cost-effective approach for patients who participate in the labour market, especially for bilateral hernias. In cost-utility analyses including quality of life (QALYs), endoscopic techniques (TEP) may be preferable since they cause less numbness and chronic pain.

Grade It is recommended that, from a hospital A perspective, an open mesh procedure is used for the treatment of inguinal hernia.

From a socio-economic perspective an endoscopic procedure is proposed for the active working population especially for bilateral hernias.

The economic aspects of inguinal hernia operations can be examined from different perspectives:

- From the perspective of the hospital accounting for the direct costs of the operation, the outpatient department visit, the stay in the hospital etc.

- From the perspective of the health insurer who funds this

- From the societal perspective in which the indirect costs of the restrictions in usual activities (e.g. time from absence from work, production losses) are also included

In 2005, McCormack et al. (Health Technology Assessment) performed a systematic review of the economic aspects of endoscopic surgery for inguinal hernia repair [198]. Fourteen studies on cost-effectiveness evaluation 
were reviewed until August 2003. TEP and TAPP were compared indirectly. A Markov model was used to perform the economic analysis. Hernia recurrence and return to work were the main outcome parameters. Also, numbness and persisting pain were included in the QALY analyses. It has been stated by others that a cost per QALY gained of $\$ 50,000$ $(=37,000 €)$ is generally viewed as a reasonable cutoff for the public funding of a medical procedure.

Endoscopic hernia was estimated to be $450-675 €$ more expensive to the health service per patient.

Unilateral hernia: in most cases, open mesh repair was the least costly option, but provided less QALYs compared to TEP or TAPP. TEP was likely to dominate TAPP.

Bilateral hernias: TEP was found to be the most costeffective in most cases, since the difference in operation times was not significant.

Recurrent hernias: the data was sparse and the results unreliable to make any conclusions on recurrent hernias. This might be a reflection of the current situation, where surgeons usually choose the endoscopic approach after open recurrence and vice versa. Thus, starting a study in this group might feel needless and ethically inappropriate.

Gholghesaei et al. [110] performed a qualitative review of 18 prospective (R)CT explicitly involving cost-effectiveness and outcome measurements associated with costs (Medline and Cochrane Central Controlled Trials Registry) in the period 1994-2004 with similar findings concluded in the Health Technology Assessment.

A very recent paper compared in 66 patients the level of postoperative pain, the use of analgetics and return to work in a RCT comparing TAPP, TEP and Lichtenstein [53]. No differences were found, except a higher operative cost for the endoscopic groups.

For the cost price of synthetic mesh, it is generally true that prefabricated products are more expensive than simple flat prostheses that can be cut to the desired shape.

Two RCTs have suggested that mesh fixation is not necessary in endoscopic surgery, provided a large prosthesis with wide overlap is used. The only exception might be large direct hernias (and femoral hernias?), although the first group used an expensive self-expandable, threedimensional prosthesis [163, 211].

Taken together, the paper by McCormack et al. gives the best overview of the current evidence, with an overall advantage for endoscopic surgery (TEP) when productivity costs and quality of life are included in the analyses (level 1B, recommendation A) [198]. Many estimations were done for primary unilateral hernia. However, data from individual trials and meta-analyses is mainly based on trials carried out in the 1990s, when the endoscopic technique was in the developing stages. For example, it has been concluded in all of the meta-analyses that endoscopic procedures take longer to perform. According to the Swedish Hernia Registry data from 2006, the mean operating times with Lichtenstein and TEP procedures were $56 \mathrm{~min}$ and $39 \mathrm{~min}$, respectively. Thus, the conclusions should be interpreted with care since local expertise, the used instrumentation and its cost can vary significantly compared to the available data. More data for bilateral and recurrent hernias are necessary.

Ideally, the total cost for Lichtenstein repair in day surgery under local anaesthesia should be compared with TEP (or TAPP) (general anaesthesia) also in day surgery, both for unilateral and bilateral/recurrent hernias. The type of employment is probably also an important determinant of indirect costs. Of course, many decisions are driven by the local health care and insurance reimbursement systems, which makes it difficult to compare studies in different European countries.

Other means to reduce direct costs are the use of reusable instruments (vs. sterilisation costs) and a shorter learning curve period with longer operation times. Therefore, a structured training programme both for open and endoscopic hernia repair is likely to be very useful. Longterm data should be investigated more closely, since, for example, chronic pain can have a significant impact on patients' quality of life and cost-effectiveness, accordingly.

Questions for the future

These guidelines provide an answer to many of the questions concerning the treatment of inguinal hernia. However, a large number of questions remain unanswered. A number of these questions can only be answered if clinical studies are performed:

- What are the late complications of mesh implantations?

- What is the best mesh?

- Does mesh cause infertility?

- Is mesh the cause of prolonged postoperative pain symptoms?

- Should inguinal hernia surgery be individualised?

- What is the precise indication area of endoscopic inguinal hernia surgery?

- How can postoperative pain be prevented?

- Is a conservative treatment for an inguinal hernia safe?

- Which diagnostic modality is the most sensitive and specific for excluding an inguinal hernia?

- What are the real risk factors for the development of an inguinal hernia?

- Are there non-operative options for treating an inguinal hernia? For example, influencing collagen synthesis? Growth factors?

- What is the best approach for teaching inguinal hernia surgery?

- Should inguinal surgery take place in specialised centres? 
- What is the relation between inguinal hernia surgery and prostatic disease?

Summary for the general practitioner

- In $95 \%$ of cases, an inguinal hernia can be diagnosed by means of a physical examination.

- Not all inguinal hernias require surgical treatment. Asymptomatic inguinal hernias (particularly in older male patients) can remain untreated.

- In female patients, the existence of a femoral hernia should be excluded in all cases of a hernia in the groin.

- It is recommended to offer patients with femoral hernia early planned surgery, even if symptoms are vague or absent.

- The risk of an inguinal hernia becoming incarcerated is less than $3 \%$ per year.

- An inguinal hernia operation can be performed adequately under local anaesthetic.

- An inguinal hernia operation can be performed on a day surgery basis, unless the comorbidity of the patient requires clinical observation.

- The use of a polypropylene prosthesis is the best technique for treating inguinal hernia. In total, $85 \%$ of operations are performed using an open approach and $15 \%$ are performed endoscopically. The surgeon should discuss the advantages and disadvantages of each technique with the patient.

- A period of rest or 'not lifting' is not necessary after an inguinal hernia operation. Patients can do what they feel capable of doing.

\section{Appendix 1: definitions and abbreviations}

Day surgery

Treatment takes place within an admission period of $10 \mathrm{~h}$. In the American literature, day surgery refers to a period of $23 \mathrm{~h}$.

Femoral hernia or hernia femoralis A protrusion of the contents of the abdominal cavity or preperitoneal adipose tissue through a hernia defect (preformed or non-preformed) in the inguinal area, below the inguinal ligament, in the lacuna vasorum, between the vena femoralis and the ligamentum lacunare (Gimbernat). This situation can lead to complaints of pain and discomfort, and can also result in incarceration.
Incarcerated inguinal hernia or hernia inguinalis incarcerata

Inguinal hernia or hernia inguinalis

Mesh prosthesis or mesh

Hernia inguinalis accreta

Recurrent inguinal hernia

Symptomatic inguinal hernia TAPP

An inguinal hernia in which the hernia sac contents have become constricted due to the narrowness of the hernia defect such that the contents can no longer be reduced and, as a result, there is a threat of intestinal obstruction and/or the blood supply to the hernia sac contents is compromised.

A protrusion of the content of the abdominal cavity through a defect (preformed or non-preformed) in the transversalis fascia above the inguinal ligament.

Literally mass or net/network; prosthesis consisting of a synthetic mesh of plastic (monofilament/ multifilament, woven/knitted, soluble/insoluble): a plastic implant used to realise a strengthening of the abdominal wall (often constructed from polypropylene, polyester or PTFE).

Inguinal hernia in which the hernia sac content can no longer be reduced without the risk of intestinal obstruction and/or causing the blood supply to the herniated part to be compromised.

A swelling (whether or not palpable during Valsalva's manoeuvre) or defect in the groin where an inguinal hernia operation has been carried out. An inguinal hernia associated with complaints and/or discomfort.

Transabdominal preperitoneal endoscopic inguinal hernia operation in which the approach to the inguinofemoral region is transabdominal, and the final placing of the prosthesis is extraperitoneal.

TEP Total extraperitoneal endoscopic inguinal hernia operation in which both the approach to the inguinofemoral region as well as the placing of the prosthesis is completely extraperitoneal. 


\section{Appendix 2: registration form}

Netherlands quality register for inguinal hernia General data

1. Hospital

2. Date form

3. Patient name

4. Date of birth

5. Hospital number

6. Gender

\section{Patient data}

1. Profession
a. Employed
b. Self-employed
c. None
d. Retired
e. Administrative
f. Manual

2. Risk factors
a. Family history
b. Long-term heavy weight lifting
c. Appendectomy
d. Smoking
e. Vascular disease
f. AAA
g. COPD
h. Prostatism
i. Constipation
j. Weight

3. How long the hernia has been present?

\section{Operation data}

1. Operation date

2. Acute

3. Antibiotics

4. Thrombosis prophylaxis

5. Anaesthesia
a. Local
b. Spinal
c. General

6. Day surgery

7. Recurrence
a. Recurrence number
b. Year last operated on
c. Technique last used

8. Bilateral

9. Contralateral inguinal hernia
10. Side

11. Non-reducible

12. Testis preoperative

13. Other intervention concurrently

14. Length of operation

15. Person performing operation
a. Staff
b. Staff + assistant
c. Assistant + staff
d. Assistant

\section{Hernia data}

1. EHS classification

2. Type
a. Direct
b. Indirect
c. Combined
d. Femoral
e. Recurrence
f. Other

3. Sliding hernia

4. Scrotal hernia

5. Exploratory pain

\section{Treatment}

1. Conservative
a. None
b. Hernia truss

2. Operative

\section{Operation technique}

1. Conventional
a. Shouldice
b. Hernia sac resection and annuloplasty
c. Bassini
d. McVay
e. Other

2. Prosthesis Anterior
a. Lichtenstein
b. Plug
c. Other

3. Prosthesis endoscopically
a. TEP
b. TAPP

\section{Postoperative complications}

1. Secondary bleeding

2. Reoperation 

3. Wound infection
4. Urine retention
5. Wound haematoma
6. Neuralgic pain
7. Reoperation due to pain
8. Vascular, intestinal or bladder damage
9. Ileus
10. Thrombosis
11. Pulmonary complication
12. Cardiac complication
13. Chronic pain
14. Death
15. Other

\section{Follow-up}

1. Months follow-up

2. Recurrence

3. Pain

4. Length of sick leave

\section{Appendix 3: operation techniques}

\section{Shouldice}

Ilio-inguinal incision. Ligation of superficial veins. Cleave external oblique (preserve ilio-inguinal nerve). Surround spermatic cord. Assess posterior wall. Cleave and ligate medial cremaster at the height of the internal ring. Cleave and ligate external spermatic vessels (not always necessary) and preserve genital branch of genito-femoral nerve. Dissect hernia sac until inside internal ring, transect, resect or reduce. Cleave fascia transversalis until in entirely healthy tissue or as far as is necessary to perform reconstruction. Reconstruction with continuous suturing using 2.0 or 3.0 polypropropylene; starting medially, not through periosteum of the pubic tubercle. Suture inferior edge of the fascia transversalis (Thomson's ligament) to a fold of the anterior side of the conjoined tendon ('white line') until the internal ring is constricted (allowing passage for the spermatic cord and point of tweezers). Return as a second layer after including cremaster stump with the same thread to the iliopubic tract (inferior edge of the inguinal ligament). Third layer begin laterally, closure of the conjoined tendon to inguinal ligament. Original Shouldice has a fourth layer in the same plane. Closure of the external oblique aponeurosis with soluble suture material without constriction of the external ring. Approximation of Scarpa's fascia. Closure of the skin.

\section{Lichtenstein}

Incision sufficiently medially for good exposure of the tubercle of pubic bone and rectus sheath. Ligation of superficial veins. Cleave external oblique (preserve ilioinguinal nerve). Surround spermatic cord. Assess posterior wall. Cremaster does not need to be excised unless hypertrophic, thus, leaving an unacceptably wide internal ring. Dissect hernia sac until inside the internal ring, transect, resect or preferably reduce. If necessary, suture a large direct hernia tension-free with continuous soluble sutures until a flat posterior wall has been created with a normal internal ring. Preserve all nerves in principle, but cut without hesitation if damaged or interference with the placement of mesh. Pay particular attention to the iliohypogastric nerve; this may lie under the mesh, but preferably not against a sharp edge (cut prosthesis to the size it needs to be; dividing a nerve is better than causing neuralgic pain). Apply polypropylene mesh $7 \times 14 \mathrm{~cm}$ (trimming is often necessary) with a $2-\mathrm{cm}$ overlap at the pubic tubercle. Suture continuously with polypropylene sutures 3.0 starting $2 \mathrm{~cm}$ mediocranially from the pubic tubercule on the lateral rectus edge and then on the inguinal ligament to the internal ring. Make an incision in the mesh on $1 / 3$ of the lower side until just medial to the spermatic cord. Suture both flaps of the prosthesis overlapping on the lateral side to the inguinal ligament with one polypropylene suture; upper flap over the lower flap. Fix cranial edge of the mesh with one or more sutures (may be soluble) to the aponeurosis of the internal oblique, avoiding muscle in order to avoid injury to the intramuscular segment of the iliohypogastric nerve. Take care not to entrap nerves by suturing! Mesh must lie tension-free (domed) after removal of the wound spreader. Close as in the Shouldice technique. In women, try to preserve the round ligament and the ilioinguinal nerve and handle in the same way as the spermatic cord. If both structures are cut, it is not necessary to create flaps in the mesh.

\section{Endoscopic (TEP)}

Anaesthetise. Bladder empty before the operation! Incision $(2 \mathrm{~cm})$ just under and next to the umbilicus until inside the anterior rectus sheath. Open preperitoneal space with the finger and, if needs be, insert balloon (optional) up to the pubic bone. Insufflation with gas under camera control. Replace balloon with blunt balloon or Hasson trocar, 10$15 \mathrm{~mm} \mathrm{Hg}$. Patient $20^{\circ}$ Trendelenburg. Identification of os pubis, Cooper's ligament, epigastric vessels and internal ring. Differentiate between direct hernia or indirect hernia. Dissect with second trocar (5 or $10 \mathrm{~mm}$ in medial line) lateral space until ASIS and insert third trocar $(5 \mathrm{~mm})$. Dissect lateral hernia sac from the spermatic cord and separate and put aside cord structures over 5-7 cm. (Via rendez-vous) insert $15 \times 15$ or $10 \times 15 \mathrm{~cm}$ polypropylene prosthesis and drape over abdominal wall with plenty of overlap for all potential hernia defects. Be aware that mesh 
edges can curl up. Carefully desufflate and remove instruments while holding the peritoneal sac 'inside' the mesh. Close the fascial defects $>10 \mathrm{~mm}$.

http://www.uzleuven.be/be/en/abdominal-surgery/ operative-procedures

\section{Appendix 4: protocol for local anaesthesia for inguinal hernias}

Amid et al. $[4,15]$.

All adult patients with an inguinal hernia (Lichtenstein, Shouldice) are eligible for an operation under local anaesthesia.

This requires a good understanding between the physician and the patient. Not every patient is suitable. Problems can arise in the case of young and very obese patients. In particular, high-risk patients are eligible. Bilateral hernias are not a contraindication.

\section{Operation:}

Low-dose benzodiazepine (usually not necessary).

Access for antibiotics, analgesics, sedatives and for calamities.

\section{Anaesthesia:}

Anaesthetist's assistant monitors the blood pressure, pulse, consciousness and circulation. He also plays an important role in supervising the patient. "Verbal anaesthesia" or Walkman.

Anaesthetist must be available for possible supportive medication and calamities.

Rarely needed.

\section{Technique:}

The surgeon is in continuous verbal contact with the patient.

Infiltration with $40-60 \mathrm{ml}$ of $50 \%$ bupivacaine $0.5 \%$, $50 \%$ lidocaine $1 \%$ if needs be with adrenaline (pay attention to blood pressure).

Maximum dosage of lidocaine $1 \%$ is $300 \mathrm{mg}$ and for bupivacaine $0.5 \% 175 \mathrm{mg}$.

No nerve block anaesthesia, but infiltration anaesthesia.

No preoperative anaesthetic. Block at anterior superior iliac spine.

\section{Local anaesthesia:}

1. Subcutaneous infiltration $5 \mathrm{ml}$.

2. Intradermal infiltration $3 \mathrm{ml}$.

3. Deep subcutaneous infiltration. Needle vertical up to the fascia in steps of $2 \mathrm{~cm}$.

4. Subcutaneous infiltration to the depth of the external oblique. Subfascial infiltration: insert needle and, in a single dose, inject $6-8 \mathrm{ml}$ in the inguinal canal. This saturates the nerves located in this canal.
5. Extra infiltration around the pubic tubercle pubicum and hernia sac.

6. Continue to anaesthetise where necessary.

\section{Appendix 5: patient information}

The text printed below has been taken from the public information texts, as compiled by the Public Information Committee of the Association of Surgeons of the Netherlands (see also http://www.heelkunde.nl).

Operation for an inguinal hernia (Hernia Inguinalis)

\section{Introduction}

This leaflet informs you about an inguinal hernia and the most usual treatment possibilities. It is worth noting that for each person the situation can be different from that described here.

\section{An inguinal hernia}

A hernia is a protrusion of the abdominal content through a weak point or opening in the abdominal wall. The hernia is recognisable as a local swelling. The hernia defect is the opening or weakening in the abdominal wall. This can arise due to congenital factors or due to stretching of the abdominal wall. Stretching can occur during the course of life, for example due to increasing body weight, straining, coughing a lot or doing a lot of heavy lifting. It is possible that the protrusion of the abdominal content-the so-called hernia sac-contains part of the abdominal contents. If the pressure on the abdomen increases (such as when standing up, straining or coughing), more of the abdominal contents can come into the protrusion (= the hernia sac). The hernia then becomes bigger.

In an inguinal hernia the protrusion is in the inguinal (or groin) area.

An inguinal hernia never disappears spontaneously and can become larger. This can lead to more complaints. Sometimes a hernia can become trapped. Then the contents of the hernia, which are mostly suddenly increased, are trapped in the hernia defect. This is very painful. An emergency operation is then necessary.

\section{Diagnosis and examination}

The surgeon establishes the diagnosis on the basis of the findings at the physical examination. Additional tests and examinations are not usually necessary. The surgeon can 
usually diagnose the hernia easily whilst you are standing up.

If you are diagnosed as having a hernia, the surgeon will discuss with you how the hernia can best be treated in your case. In general an operation will be advised. A hernia truss is only prescribed very rarely nowadays.

\section{The operation}

Depending on the circumstances, the operation can be carried out as day surgery or during a short hospital admission. The anaesthetist will discuss with you whether the operation will take place under a spinal, general or local anaesthetic.

There are various techniques to repair inguinal hernias. Two principle methods are used:

- Approaching the hernia from the front. In this the operation is performed via a cut close to the hernia. The protrusion of the abdominal wall is removed. If necessary the opening or weak point in the abdominal wall is repaired. During this the abdominal wall is strengthened, making use of the tissue from the abdominal wall itself (termed herniorrhaphy) or by stitching in a piece of synthetic material. This synthetic material is safe and is usually well-tolerated by the body.

- Approaching the hernia from behind. In this method the hernia is treated from the inside of the abdominal wall. The protrusion (hernia sac) is removed and the opening or weak point in the abdominal wall is strengthened by means of a piece of synthetic material. The synthetic material is safe and is usually well-tolerated by the body. The operative approach of the hernia from the inside can be carried out by means of conventional or keyhole surgery techniques. In keyhole surgery the instruments and a camera are inserted via small holes in the abdomen. The camera is linked with a TV monitor. Via the camera the surgeon can see what he is doing on the TV screen.

These new methods are not suitable for every patient. For example, if the hernia cannot be pushed back then this method cannot be used.

The surgeon will discuss with you which method seems best in your case. An inguinal hernia operation usually takes $45 \mathrm{~min}$ to one hour to perform.

\section{Possible complications}

No operation is free of risks. In these operations the normal risk of complications is also present, such as secondary bleeding, wound infections, thrombosis or pneumonia.
You can recognise a minor expression of a bleeding after several days in the form of a blue discolouration in the wound area, which can spread down into the base of the penis and scrotum in men or into the labium majora in women. This is not a reason for concern.

The result of the operation might seem to be good. Yet during the course of time a small number of patients who have been operated on can develop a hernia in the same place (a recurrent hernia). In such cases another operation is usually necessary.

As there are several nerves in the area operated on-in men also the spermatic cord-damage to these structures might occur. Fortunately, such complications rarely occur. A loss of feeling or sometimes a continuous pain around the operation area can occur as a result of damage to a nerve.

\section{After the operation}

After the operation, the operation area will be painful. You can use painkillers such as paracetamol for the pain. You can buy these from a pharmacist or chemist beforehand so that you already have these painkillers at home prior to the operation.

Shortly after the operation it is often advisable to support the wound area with your hand, especially when the pressure increases (coughing, straining).

Depending on the operation method, the size of the operation and individual factors, you may experience inconvenience in the operation area for a while after you have been discharged. Also the resumption of your daily activities and the possibility of lifting things again will depend on this. The surgeon will provide you with some advice concerning this.

\section{Discharge}

Upon discharge you will be given an appointment for an outpatients' check-up. The stitches can be removed after a week. This can be done by the general practitioner or during the outpatients' check-up. Sometimes use is made of soluble stitches, which do not need to be removed.

\section{Questions}

If you still have questions, please direct these to the treating surgeon or your general practitioner.

In the case of urgent questions or problems prior to your treatment you can best contact the department where the treatment will take place. If problems occur at home after the operation, please contact your general practitioner or the hospital. 


\section{Conclusion}

If you are of the opinion that certain information is lacking or unclear, please could you be so kind as to inform us.

\section{Appendix 6: result of AGREE}

\section{European Hernia Society Guidelines Treatment of inguinal hernia in adult patients Objective}

To support the daily practice of the treatment of inguinal hernia by surgeons. The guidelines are intended as a reference manual.

\section{Target population}

All patients with a primary or recurrent inguinal hernia (asymptomatic or symptomatic, acute or elective). The guidelines concern male patients unless stated otherwise.

\section{Intended (target) users}

Surgeons and trainee surgeons. Some chapters are also intended for other care providers, such as general practitioners.

\section{Initial questions}

a. What are the indications for inguinal hernia treatment? Is operative treatment necessary?

b. What is the best technique for the treatment of an inguinal hernia (considering factors like recurrence, complications, postoperative recovery, pain, costs)? What mesh is best?

c. What are the complications of the various techniques, and how can these be treated? What causes pain complications and how to treat these?

d. What is the best form of anaesthetic? Should local anaesthesia be recommended as the first choice?

e. Can an inguinal hernia be operated in ambulatory surgery? Thus, decreasing cost, possibly improving quality?

f. Is the routine use of antibiotics necessary?

\section{Specific questions}

1. What are the indications for a surgical treatment of inguinal hernia? Can a non-surgical (conservative) treatment be considered?

2. Which diagnostic modality is the most suitable for diagnosing inguinal hernia in patients with groin complaints (without clear swelling in the groin region)?

3. Is it necessary to classify inguinal hernias and which classification is the most suitable?

4. What are the risk factors for developing an inguinal hernia and are there preventive measures?
5. What is the best technique for treating an inguinal hernia, taking into account the type of hernia and the patient?

6. Following a non-mesh inguinal hernia operation, is the risk of recurrence lower for women than for men? Should women be treated with a different strategy?

7. Does a young man have a very low risk of recurrence following a non-mesh inguinal hernia operation due to an indirect hernia? Is mesh treatment indicated for this category of patients?

8. What mesh type is the most suitable in inguinal hernia repair, and what mesh-related complications can occur?

9. Can inguinal hernia surgery be performed in a day surgery setting? Is this safe and cost-effective?

10. Is antibiotic prophylaxis routinely indicated for elective inguinal surgery for primary inguinal hernia?

11. What is the learning curve and training in inguinal hernia repair?

12. Can an open inguinal hernia operation under local anaesthesia be performed with the same patient satisfaction? Is this safer and more cost-effective than other anaesthesia techniques? Should regional anaesthesia be avoided?

13. Which technique gives the fastest postoperative recovery?

14. Is a lifting, sports or work ban indicated following inguinal hernia surgery?

15. What is the best method for realising an effective postoperative pain control?

16. How frequent are complications after inguinal hernia operations, and can the risk of complications be reduced? Which are the specific complications following inguinal hernia operation and how should they be treated?

17. What is the most cost-effective operation for the treatment of primary inguinal hernia?

\section{Assessment of the guidelines}

We assessed the guidelines by using the Appraisal of Guidelines for REsearch and Evaluation (AGREE) instrument, version September 2001. A previous version of the guidelines (August 2008) was assessed independently by two appraisers. Differences in scores were discussed to reach consensus. For scores lower than 4 ('strongly agree'), we explained the reasons for our response. Apart from the AGREE assessment, we had some other comments and suggestions to improve the quality of the guidelines.

We discussed our assessment and comments with the guidelines' authors. Many adjustments were made. The next version of the guidelines (February 2009) was reassessed with AGREE by one appraiser and discussed with the second appraiser. 
We did not calculate standardised domain scores. Although the domain scores may be useful for comparing guidelines and will inform the decision as to whether or not to use or recommend a guideline, it is not possible to set thresholds to mark a 'good' or 'bad' guideline.

\section{AGREE assessment}

Score: $1=$ strongly disagree, $2=$ disagree, $3=$ agree, $4=$ strongly agree

Table 1

Item

Scope and purpose

1 The overall objective(s) of the guidelines is (are) specifically 4 described

2 The clinical question(s) covered by the guideline is (are) specifically described

3 The patients to whom the guideline is meant to apply are 4 specifically described

Stakeholder involvement

4 The guideline development group includes individuals from 3 all of the relevant professional groups

"These guidelines are primarily intended for surgeons and trainee surgeons

Some chapters are also intended for other care providers such as general practitioners, who wish to provide information to patients with an inguinal hernia"

General practitioners were not part of the steering or Working Group

5 The patients' views and preferences have been sought

Needs to be written

6 The target users of the guidelines are clearly defined

7 The guideline has been piloted among target users

"For the Dutch Guidelines that were published in 2003 an implementation study and a pilot study among targeted users were performed"

It is not clear if this pilot is still relevant

Rigour of development

8 Systematic methods were used to search for evidence

"All relevant literature until April 2007 (Medline, Embase and Cochrane) was prepared by small groups and assessed by all Working Group members. Literature of all level 1A and/or 1B studies was searched during the development of the Guidelines until May 2008"

The databases and time frames are described; the description of the search terms is limited

9 The criteria for selecting the evidence are clearly described 1

"For all articles, in accordance with evidence-based guidelines criteria, two surgeons always determined whether or not an article was relevant (according to possible bias). Each time a unanimous final opinion was sought and this was always realised. The Working Group met on 3 occasions. For chapters in which only level $2 \mathrm{c}$ or 3 articles were available it was difficult to choose best evidence from at times hundreds of articles. Search bias in these cases cannot be excluded"

Criteria and reasons for including and excluding studies are not mentioned (e.g. language or publication type restriction, exclusion of low quality studies)

"According to evidence based medicine guidelines quality was assessed"

The method of assessment of the quality of the studies is not clear (e.g. which methodological items were assessed)
Table 1 continued

10 The methods used for formulating the recommendations are 2 clearly described

"The concept chapters were discussed and (where necessary) consensus was found after which recommendations were agreed upon"

"After this a consensus (where necessary) was reached and the conclusions and recommendations were formulated"

The description of the methods used for formulating the recommendations is limited. For example, were other factors than evidence from the literature taken into consideration? If so, what were these other factors and how where they weighted against the scientific evidence?

The classification for diagnostic studies is not mentioned

Evidence tables are missing

11 The health benefits, side effects and risks have been considered in formulating the recommendations

The effects of the recommendations (health benefits, side effects, risks) are not mentioned; however, some questions address some of the effects (e.g. complications, Chap. 2.17)

12 There is an explicit link between the recommendation and the 2 supporting evidence

AGREE recommends that each recommendation should be linked with a list of references on which it is based

13 The guideline has been externally reviewed by experts prior 3 to publication

"The Appraisal of Guidelines for REsearch \& Evaluation (AGREE) instrument was used to validate the Guidelines

Almost all criteria were fulfilled. Review was performed by four external experts in surgery and epidemiology. Two members of the Dutch Cochrane Institute performed a rigorous analysis which led to many adjustments"

Did the reviewers use AGREE also? How was the review done?

Which criteria were not fulfilled?

14 A procedure for updating the guideline is provided

"The guidelines are valid until 1 January 2011. Update of guidelines (literature) will be performed continuously by the two authors of each chapter with a yearly meeting at the EHS at which publication of relevant updates will be decided upon"

This seems contradictory: the guidelines are valid until 2011, but are updated yearly?

Clarity and presentation

15 The recommendations are specific and unambiguous 3

16 The different options for management of the condition are 4 clearly presented

17 Key recommendations are easily identifiable

18 The guideline is supported with tools for application

"The EHS is developing a skills and teaching institute to facilitate and train surgeons and residents to be able to work according to the guidelines"

A summary of the conclusions and recommendations, a flow chart and patient information are available, educational tools are under development

Applicability

19 The potential organisational barriers in applying the recommendations have been discussed

"A pilot study among targeted users was performed in two large district hospitals in The Netherlands in 2002. There were no barriers to implementation either in costs or logistical possibilities. There are possibly European Countries where certain hospitals cannot afford endoscopic surgery"

Investigation of the barriers was based on the pilot in 2002 
Table 1 continued

\begin{tabular}{l}
\hline 20 The potential costs implications of applying the \\
recommendations have been considered \\
"A pilot study among targeted users was performed in two large \\
district hospitals in The Netherlands in 2002. There were no \\
barriers to implementation either in costs or logistical \\
possibilities. There are possibly European Countries where \\
certain hospitals cannot afford endoscopic surgery" \\
Investigation of the barriers was based on the pilot in 2002 \\
The guideline presents key review criteria for monitoring \\
and/or audit purposes \\
Registration is under development \\
Editorial comments \\
The guideline is editorially independent from the funding \\
body \\
Development of the guideline was financed through a grant by \\
Ethicon, a medical device company. Ethicon would not \\
become owner and would not interfere with the methods and \\
contents. We are not able to know if the recommendations are \\
completely independent from the sponsor \\
Conflicts of interest of guideline development members have 3 \\
been recorded \\
Members with no conflicts of interest were sought. However, \\
conflicts of interest were not recorded
\end{tabular}

Open Access This article is distributed under the terms of the Creative Commons Attribution Noncommercial License which permits any noncommercial use, distribution, and reproduction in any medium, provided the original author(s) and source are credited.

\section{References}

1. Aasvang E, Kehlet H (1986) Classification of chronic pain. Descriptions of chronic pain syndromes and definitions of pain terms. Prepared by the International Association for the Study of Pain, Subcommittee on Taxonomy. Pain Suppl 3:S1-S226

2. Aasvang E, Kehlet H (2005) Chronic postoperative pain: the case of inguinal herniorrhaphy. Br J Anaesth 95:69-76

3. Aasvang E, Kehlet H (2005) Surgical management of chronic pain after inguinal hernia repair. Br J Surg 92:795-801

4. Aasvang EK, Møhl B, Bay-Nielsen M, Kehlet H (2006) Pain related sexual dysfunction after inguinal herniorrhaphy. Pain 122:258-263

5. Aasvang EK, Møhl B, Kehlet H (2007) Ejaculatory pain: a specific postherniotomy pain syndrome? Anesthesiology 107:298-304

6. Abe T, Shinohara N, Harabayashi T, Sazawa A, Suzuki S, Kawarada Y, Nonomura K (2007) Postoperative inguinal hernia after radical prostatectomy for prostate cancer. Urology 69:326329

7. Adamonis W, Witkowski P, Smietański M, Bigda J, Sledziński Z (2006) Is there a need for a mesh plug in inguinal hernia repair? Randomized, prospective study of the use of Hertra 1 mesh compared to PerFix Plug. Hernia 10:223-228

8. Agrawal A, Avill R (2006) Mesh migration following repair of inguinal hernia: a case report and review of literature. Hernia 10:79-82

9. Akpinar E, Turkbey B, Ozcan O, Akdogan B, Karcaaltincaba M, Ozen H (2005) Bilateral scrotal extraperitoneal herniation of ureters: computed tomography urographic findings and review of the literature. J Comput Assist Tomogr 29:790-792
10. Alam A, Nice C, Uberoi R (2005) The accuracy of ultrasound in the diagnosis of clinically occult groin hernias in adults. Eur Radiol 15:2457-2461

11. Alsarrage SAM, Godbole CSM (1990) A randomised controlled trial to compare local with general anaesthesia for inguinal hernia repair. J Kuwait Med Assoc 24:31-34

12. Alvarez JA, Baldonedo RF, Bear IG, Solís JA, Alvarez P, Jorge JI (2004) Incarcerated groin hernias in adults: presentation and outcome. Hernia 8:121-126

13. Amid PK (2000) Driving after repair of groin hernia. BMJ 321:1033-1034

14. Amid PK (2004) Causes, prevention, and surgical treatment of postherniorrhaphy neuropathic inguinodynia: triple neurectomy with proximal end implantation. Hernia 8:343-349

15. Amid PK, Shulman AG, Lichtenstein IL (1994) Local anesthesia for inguinal hernia repair step-by-step procedure. Ann Surg 220:735-737

16. Amid PK, Shulman AG, Lichtenstein IL (1994) Local anesthesia for inguinal hernia repair step-by-step procedure. Ann Surg 220:735-737

17. Amid PK, Shulman AG, Lichtenstein IL (1996) Open "tensionfree" repair of inguinal hernias: the Lichtenstein technique. Eur J Surg 162:447-453

18. Andac N, Baltacioğlu F, Tüney D, Cimşit NC, Ekinci G, Biren T (2002) Inguinoscrotal bladder herniation: is CT a useful tool in diagnosis? Clin Imaging 26:347-348

19. Andersen JR, Burcharth F, Larsen HW, Røder O, Andersen B (1980) Polyglycolic acid, silk, and topical ampicillin. Their use in hernia repair and cholecystectomy. Arch Surg 115:293-295

20. Arvidsson D, Smedberg S (2000) Laparoscopic compared with open hernia surgery: complications, recurrences and current trends. Eur J Surg Suppl 585:40-47

21. Arvidsson D, Berndsen FH, Larsson LG, Leijonmarck CE, Rimbäck G, Rudberg C, Smedberg S, Spangen L, Montgomery A (2005) Randomized clinical trial comparing 5-year recurrence rate after laparoscopic versus Shouldice repair of primary inguinal hernia. Br J Surg 92:1085-1091

22. Aufenacker TJ, van Geldere D, van Mesdag T, Bossers AN, Dekker B, Scheijde E, van Nieuwenhuizen R, Hiemstra E, Maduro JH, Juttmann JW, Hofstede D, van Der Linden CT, Gouma DJ, Simons MP (2004) The role of antibiotic prophylaxis in prevention of wound infection after Lichtenstein open mesh repair of primary inguinal hernia: a multicenter doubleblind randomized controlled trial. Ann Surg 240:955-960

23. Aufenacker TJ, Koelemay MJ, Gouma DJ, Simons MP (2006) Systematic review and meta-analysis of the effectiveness of antibiotic prophylaxis in prevention of wound infection after mesh repair of abdominal wall hernia. Br J Surg 93:5-10

24. Aufenacker TJ, Schmits SP, Gouma DJ, Simons MP (2009) Do guidelines influence results in inguinal hernia treatment? A descriptive study of 2,535 hernia repairs in one teaching hospital from 1994 to 2004. Hernia 13:35-39

25. Baker DM, Rider MA, Fawcett AN (1994) When to return to work following a routine inguinal hernia repair: are doctors giving the correct advice? J R Coll Surg Edinb 39:31-33

26. Balducci D, Postiglione V, Pancaldi R, Lippa M, Tonni MP, Morandi O, Mazzetti S, Beschi M, Diana DR, Vergallo A, Bruni GC (1997) Prosthetic repair of inguinal and crural hernias. Minerva Chir 52:1069-1075

27. Barbier J, Carretier M, Richer JP (1989) Cooper ligament repair: an update. World J Surg 13:499-505

28. Barile A, Erriquez D, Cacchio A, De Paulis F, Di Cesare E, Masciocchi C (2000) Groin pain in athletes: role of magnetic resonance. Radiol Med (Torino) 100:216-222

29. Barrat C, Voreux JP, Occelli G, Catheline JM, Champault G (1999) Effects of surgical education and training on the results 
of laparoscopic treatment of inguinal hernias. Chirurgie 124:298-303

30. Bay-Nielsen M, Kehlet $H$ (2006) Inguinal herniorrhaphy in women. Hernia 10:30-33

31. Bay-Nielsen M, Knudsen MS, Christensen JK, Kehlet H (1999) Cost analysis of inguinal hernia surgery in Denmark. Ugeskr Laeger 161:5317-5321

32. Bay-Nielsen M, Kehlet H, Strand L, Malmstrøm J, Andersen FH, Wara P, Juul P, Callesen T; Danish Hernia Database Collaboration (2001) Quality assessment of 26,304 herniorrhaphies in Denmark: a prospective nationwide study. Lancet 358:11241128

33. Bay-Nielsen M, Nilsson E, Nordin P, Kehlet H; Swedish Hernia Data Base the Danish Hernia Data Base (2004) Chronic pain after open mesh and sutured repair of indirect inguinal hernia in young males. Br J Surg 91:1372-1376

34. Beacon J, Hoile RW, Ellis H (1980) A trial of suction drainage in inguinal hernia repair. Br J Surg 67:554-555

35. Beets GL, van Geldere D, Baeten CG, Go PM (1996) Long-term results of giant prosthetic reinforcement of the visceral sac for complex recurrent inguinal hernia. Br J Surg 83:203-206

36. Beets GL, Oosterhuis KJ, Go PM, Baeten CG, Kootstra G (1997) Longterm followup (12-15 years) of a randomized controlled trial comparing Bassini-Stetten, Shouldice, and high ligation with narrowing of the internal ring for primary inguinal hernia repair. J Am Coll Surg 185:352-357

37. Behnia R, Hashemi F, Stryker SJ, Ujiki GT, Poticha SM (1992) A comparison of general versus local anesthesia during inguinal herniorrhaphy. Surg Gynecol Obstet 174:277-280

38. Berndsen FH, Petersson U, Arvidsson D, Leijonmarck CE, Rudberg C, Smedberg S, Montgomery A; SMIL Study Group (2007) Discomfort five years after laparoscopic and Shouldice inguinal hernia repair: a randomised trial with 867 patients. A report from the SMIL study group. Hernia 11:307-313

39. Birkmeyer JD, Stukel TA, Siewers AE, Goodney PP, Wennberg DE, Lucas FL (2003) Surgeon volume and operative mortality in the United States. N Engl J Med 349:2117-2127

40. Bittner R, Schmedt CG, Schwarz J, Kraft K, Leibl BJ (2002) Laparoscopic transperitoneal procedure for routine repair of groin hernia. Br J Surg 89:1062-1066

41. Bittner R, Sauerland S, Schmedt CG (2005) Comparison of endoscopic techniques vs Shouldice and other open nonmesh techniques for inguinal hernia repair: a meta-analysis of randomized controlled trials. Surg Endosc 19:605-615

42. Bolognini S, Orsini V, Grandinetti PP, Pata F, Sacco R (2006) Lichtenstein vs. Rutkow-Robbins technique in the treatment of primary inguinal hernia. Analysis of the long term results. Ann Ital Chir 77:51-56

43. Borenstein SH, To T, Wajja A, Langer JC (2005) Effect of subspecialty training and volume on outcome after pediatric inguinal hernia repair. J Pediatr Surg 40:75-80

44. Bowley DM, Butler M, Shaw S, Kingsnorth AN (2003) Dispositional pessimism predicts delayed return to normal activities after inguinal hernia operation. Surgery 133:141-146

45. Bradley M, Morgan D, Pentlow B, Roe A (2003) The groin hernia-an ultrasound diagnosis? Ann R Coll Surg Engl $85: 178-180$

46. Bringman S, Ramel S, Heikkinen TJ, Englund T, Westman B, Anderberg B (2003) Tension-free inguinal hernia repair: TEP versus mesh-plug versus Lichtenstein: a prospective randomized controlled trial. Ann Surg 237:142-147

47. Bringman S, Heikkinen TJ, Wollert S, Osterberg J, Smedberg S, Granlund H, Ramel S, Felländer G, Anderberg B (2004) Early results of a single-blinded, randomized, controlled, Internetbased multicenter trial comparing Prolene and Vypro II mesh in Lichtenstein hernioplasty. Hernia 8:127-134
48. Bringman S, Wollert S, Osterberg J, Heikkinen T (2005) Early results of a randomized multicenter trial comparing Prolene and VyproII mesh in bilateral endoscopic extraperitoneal hernioplasty (TEP). Surg Endosc 19:536-540

49. Bringman S, Wollert S, Osterberg J, Smedberg S, Granlund H, Felländer G, Heikkinen T (2005) One year results of a randomised controlled multi-centre study comparing Prolene and Vypro II-mesh in Lichtenstein hernioplasty. Hernia 9:223-227

50. Bringman S, Wollert S, Osterberg J, Smedberg S, Granlund H, Heikkinen TJ (2006) Three-year results of a randomized clinical trial of lightweight or standard polypropylene mesh in Lichtenstein repair of primary inguinal hernia. Br J Surg 93:10561059

51. Browne J, Murphy D, Shorten G (2000) Pneumomediastinum, pneumothorax and subcutaneous emphysema complicating MIS herniorrhaphy. Can J Anaesth 47:69-72

52. Burney RE, Prabhu MA, Greenfield ML, Shanks A, O'Reilly M (2004) Comparison of spinal vs general anesthesia via laryngeal mask airway in inguinal hernia repair. Arch Surg 139:183-187

53. Butler RE, Burke R, Schneider JJ, Brar H, Lucha PA Jr (2007) The economic impact of laparoscopic inguinal hernia repair: results of a double-blinded, prospective, randomized trial. Surg Endosc 21:387-390

54. Butters M, Redecke J, Köninger J (2007) Long-term results of a randomized clinical trial of Shouldice, Lichtenstein and transabdominal preperitoneal hernia repairs. Br J Surg 94:562-565

55. Calder F, Evans R, Neilson D, Hurley P (2000) Value of herniography in the management of occult hernia and chronic groin pain in adults. Br J Surg 87:824-825

56. Callesen $T$ (2003) Inguinal hernia repair: anaesthesia, pain and convalescence. Dan Med Bull 50:203-218

57. Callesen T, Bech K, Kehlet H (1998) The feasibility, safety and cost of infiltration anaesthesia for hernia repair. Hvidovre Hospital Hernia Group. Anaesthesia 53:31-35

58. Callesen T, Klarskov B, Bech K, Kehlet H (1999) Short convalescence after inguinal herniorrhaphy with standardised recommendations: duration and reasons for delayed return to work. Eur J Surg 165:236-241

59. Callesen T, Bech K, Kehlet H (2001) One-thousand consecutive inguinal hernia repairs under unmonitored local anesthesia. Anesth Analg 93:1373-1376

60. Campanelli G, Pettinari D, Nicolosi FM, Cavalli M, Avesani EC (2006) Inguinal hernia recurrence: classification and approach. Hernia 10:159-161

61. Cannon DJ, Read RC (1981) Metastatic emphysema: a mechanism for acquiring inguinal herniation. Ann Surg 194:270278

62. Carbonell JF, Sanchez JL, Peris RT, Ivorra JC, Del Baño MJ, Sanchez CS, Arraez JI, Greus PC (1993) Risk factors associated with inguinal hernias: a case control study. Eur J Surg 159:481486

63. Caterino M, Finocchi V, Giunta S, De Carli P, Crecco M (2001) Bladder cancer within a direct inguinal hernia: CT demonstration. Abdom Imaging 26:664-666

64. Celdrán A, Frieyro O, de la Pinta JC, Souto JL, Esteban J, Rubio JM, Señarís JF (2004) The role of antibiotic prophylaxis on wound infection after mesh hernia repair under local anesthesia on an ambulatory basis. Hernia 8:20-22

65. Chiu AW, Chang LS, Birkett DH, Babayan RK (1995) The impact of pneumoperitoneum, pneumoretroperitoneum, and gasless laparoscopy on the systemic and renal hemodynamics. J Am Coll Surg 181:397-406

66. Chowbey PK, Bagchi N, Goel A, Sharma A, Khullar R, Soni V, Baijal M (2006) Mesh migration into the bladder after TEP repair: a rare case report. Surg Laparosc Endosc Percutan Tech $16: 52-53$ 
67. Chuback JA, Singh RS, Sills C, Dick LS (2000) Small bowel obstruction resulting from mesh plug migration after open inguinal hernia repair. Surgery 127:475-476

68. Chung RS, Rowland DY (1999) Meta-analyses of randomized controlled trials of laparoscopic vs conventional inguinal hernia repairs. Surg Endosc 13:689-694

69. Coates KW, Kuehl TJ, Bachofen CG, Shull BL (2001) Analysis of surgical complications and patient outcomes in a residency training program. Am J Obstet Gynecol 184:1380-1383

70. Collaboration EH (2000) Laparoscopic compared with open methods of groin hernia repair: systematic review of randomized controlled trials. Br J Surg 87:860-867

71. Collaboration EH (2000) Mesh compared with non-mesh methods of open groin hernia repair: systematic review of randomized controlled trials. Br J Surg 87:854-859

72. Collaboration EH (2002) Repair of groin hernia with synthetic mesh: meta-analysis of randomized controlled trials. Ann Surg 235:322-332

73. Courtney CA, Duffy K, Serpell MG, O’Dwyer PJ (2002) Outcome of patients with severe chronic pain following repair of groin hernia. Br J Surg 89:1310-1314

74. Cueto Rozon R, De Baerdemacker Y, Polliand C, Champault G (2006) Surgical training and inguinal hernia repair. Ann Chir 131:311-315

75. Davies BW, Campbell WB (1995) Inguinal hernia repair: see one, do one, teach one? Ann R Coll Surg Engl 77:299-301

76. Davies KE, Houghton K, Montgomery JE (2001) Obesity and day-case surgery. Anaesthesia 56:1112-1115

77. De Lathouwer C, Poullier JP (2000) How much ambulatory surgery in the World in 1996-1997 and trends? Ambul Surg 8:191-210

78. Dedemadi G, Sgourakis G, Karaliotas C, Christofides T, Kouraklis G, Karaliotas C (2006) Comparison of laparoscopic and open tension-free repair of recurrent inguinal hernias: a prospective randomized study. Surg Endosc 20:1099-1104

79. DeTurris SV, Cacchione RN, Mungara A, Pecoraro A, Ferzli GS (2002) Laparoscopic herniorrhaphy: beyond the learning curve. J Am Coll Surg 194:65-73

80. Devlin HB, Kingsnorth AN (1998) Management of abdominal hernias. Chapman and Hall Medical, London

81. Dierking GW, Ostergaard E, Ostergård HT, Dahl JB (1994) The effects of wound infiltration with bupivacaine versus saline on postoperative pain and opioid requirements after herniorrhaphy. Acta Anaesthesiol Scand 38:289-292

82. Dirksen CD, Beets GL, Go PM, Geisler FE, Baeten CG, Kootstra G (1998) Bassini repair compared with laparoscopic repair for primary inguinal hernia: a randomised controlled trial. Eur J Surg 164:439-447

83. Dogru O, Girgin M, Bulbuller N, Cetinkaya Z, Aygen E, Camci C (2006) Comparison of Kugel and Lichtenstein operations for inguinal hernia repair: results of a prospective randomized study. World J Surg 30:346-350

84. Douek M, Smith G, Oshowo A, Stoker DL, Wellwood JM (2003) Prospective randomised controlled trial of laparoscopic versus open inguinal hernia mesh repair: five year follow up. BMJ 326:1012-1013

85. Dudda W, Schunk R (1990) Lotheissen-McVay repair of hernia. Late follow-up analysis after 1202 operations for inguinal and femoral hernias. Langenbecks Arch Chir 375:351-358

86. Edwards CC 2nd, Bailey RW (2000) Laparoscopic hernia repair: the learning curve. Surg Laparosc Endosc Percutan Tech 10:149-153

87. Eklund A, Rudberg C, Smedberg S, Enander LK, Leijonmarck CE, Osterberg J, Montgomery A (2006) Short-term results of a randomized clinical trial comparing Lichtenstein open repair with totally extraperitoneal laparoscopic inguinal hernia repair. Br J Surg 93:1060-1068

88. Eklund A, Rudberg C, Leijonmarck CE, Rasmussen I, Spangen L, Wickbom G, Wingren U, Montgomery A (2007) Recurrent inguinal hernia: randomized multicenter trial comparing laparoscopic and Lichtenstein repair. Surg Endosc 21:634-640

89. Engbaek J, Bartholdy J, Hjorts $\varnothing$ NC (2006) Return hospital visits and morbidity within 60 days after day surgery: a retrospective study of 18,736 day surgical procedures. Acta Anaesthesiol Scand 50:911-919

90. Engeset J, Youngson GG (1984) Ambulatory peritoneal dialysis and hernial complications. Surg Clin North Am 64:385-392

91. Eubanks S, Newman L 3rd, Goehring L, Lucas GW, Adams CP, Mason E, Duncan T (1993) Meralgia paresthetica: a complication of laparoscopic herniorrhaphy. Surg Laparosc Endosc 3:381-385

92. Evans C, Pollock AV (1973) The reduction of surgical wound infections by prophylactic parenteral cephaloridine. A controlled clinical trial. Br J Surg 60:434-437

93. Fallon WF Jr, Wears RL, Tepas JJ 3rd (1993) Resident supervision in the operating room: does this impact on outcome? J Trauma 35:556-560

94. Farquharson EL (1955) Early ambulation; with special reference to herniorrhaphy as an outpatient procedure. Lancet 269:517519

95. Feliu-Palà X, Martín-Gómez M, Morales-Conde S, FernándezSallent E (2001) The impact of the surgeon's experience on the results of laparoscopic hernia repair. Surg Endosc 15:1467-1470

96. Felix EL, Harbertson N, Vartanian S (1999) Laparoscopic hernioplasty: significant complications. Surg Endosc 13:328-331

97. Ferzli GS, Kiel T, Hurwitz JB, Davidson P, Piperno B, Fiorillo MA, Hayek NE, Riina LL, Sayad P (1997) Pneumothorax as a complication of laparoscopic inguinal hernia repair. Surg Endosc 11:152-153

98. Finley RK Jr, Miller SF, Jones LM (1991) Elimination of urinary retention following inguinal herniorrhaphy. Am Surg $57: 486-488$

99. Fitzgibbons RJ Jr, Camps J, Cornet DA, Nguyen NX, Litke BS, Annibali R, Salerno GM (1995) Laparoscopic inguinal herniorrhaphy. Results of a multicenter trial. Ann Surg 221:3-13

100. Fitzgibbons RJ Jr, Giobbie-Hurder A, Gibbs JO, Dunlop DD, Reda DJ, McCarthy M Jr, Neumayer LA, Barkun JS, Hoehn JL, Murphy JT, Sarosi GA Jr, Syme WC, Thompson JS, Wang J, Jonasson O (2006) Watchful waiting vs repair of inguinal hernia in minimally symptomatic men: a randomized clinical trial. JAMA 295:285-292

101. Flich J, Alfonso JL, Delgado F, Prado MJ, Cortina P (1992) Inguinal hernia and certain risk factors. Eur J Epidemiol 8:277282

102. Foschi D, Corsi F, Cellerino P, Trabucchi A, Trabucchi E (1998) Late rejection of the mesh after laparoscopic hernia repair. Surg Endosc 12:455-457

103. Frey DM, Wildisen A, Hamel CT, Zuber M, Oertli D, Metzger J (2007) Randomized clinical trial of Lichtenstein's operation versus mesh plug for inguinal hernia repair. Br J Surg 94:3641

104. Friemert B, Faoual J, Hölldobler G, Becker HP, Lampl L, Gerngross H (2000) A prospective randomized study on inguinal hernia repair according to the Shouldice technique. Benefits of local anesthesia. Chirurg 71:52-57

105. Friis E, Lindahl F (1996) The tension-free hernioplasty in a randomized trial. Am J Surg 172:315-319

106. Fuchsjäger N, Feichter A, Hirbawi A, Kux M (1989) Bassini operation with polyglycolic acid or polyester. A prospective randomized study of 300 cases. Chirurg 60:273-276 
107. Gallegos NC, Dawson J, Jarvis M, Hobsley M (1991) Risk of strangulation in groin hernias. Br J Surg 78:1171-1173

108. Garner JP, Patel S, Glaves J, Ravi K (2006) Is herniography useful? Hernia 10:66-69

109. Gatt MT, Chevrel JP (1991) Treatment of neuralgia after surgical repair of inguinal hernia. Apropos of 47 cases. Chirurgie 117:96-103

110. Gholghesaei M, Langeveld HR, Veldkamp R, Bonjer HJ (2005) Costs and quality of life after endoscopic repair of inguinal hernia vs open tension-free repair: a review. Surg Endosc $19: 816-821$

111. Go PMNYH, Rutten CLG, Grasveld-van Berkel MA, van Montfort APWP (2002) Dagbehandeling in Nederland. Lemma, Utrecht

112. Godfrey PJ, Greenan J, Ranasinghe DD, Shabestary SM, Pollock AV (1981) Ventilatory capacity after three methods of anaesthesia for inguinal hernia repair: a randomized controlled trial. Br J Surg 68:587-589

113. Gönüllü NN, Cubukçu A, Alponat A (2002) Comparison of local and general anesthesia in tension-free (Lichtenstein) hernioplasty: a prospective randomized trial. Hernia 6:29-32

114. Goulbourne IA, Ruckley CV (1979) Operations for hernia and varicose veins in a day-bed unit. Br Med J 2:712-714

115. Grant AM; EU Hernia Trialists Collaboration (2002) Laparoscopic versus open groin hernia repair: meta-analysis of randomised trials based on individual patient data. Hernia 6:210

116. Grant AM; EU Hernia Trialists Collaboration (2002) Open mesh versus non-mesh repair of groin hernia: meta-analysis of randomised trials based on individual patient data [corrected]. Hernia 6:130-136

117. Grant AM, Scott NW, O’Dwyer PJ; MRC Laparoscopic Groin Hernia Trial Group (2004) Five-year follow-up of a randomized trial to assess pain and numbness after laparoscopic or open repair of groin hernia. Br J Surg 91:1570-1574

118. Gultekin FA, Kurukahvecioglu O, Karamercan A, Ege B, Ersoy E, Tatlicioglu E (2007) A prospective comparison of local and spinal anesthesia for inguinal hernia repair. Hernia 11:153-156

119. Gwanmesia II, Walsh S, Bury R, Bowyer K, Walker S (2001) Unexplained groin pain: safety and reliability of herniography for the diagnosis of occult hernias. Postgrad Med J 77:250-251

120. Haidenberg J, Kendrick ML, Meile T, Farley DR (2003) Totally extraperitoneal (TEP) approach for inguinal hernia: the favorable learning curve for trainees. Curr Surg 60:65-68

121. Hair A, Duffy K, McLean J, Taylor S, Smith H, Walker A, MacIntyre IM, O'Dwyer PJ (2000) Groin hernia repair in Scotland. Br J Surg 87:1722-1726

122. Hair A, Paterson C, Wright D, Baxter JN, O’Dwyer PJ (2001) What effect does the duration of an inguinal hernia have on patient symptoms? J Am Coll Surg 193:125-129

123. Hall C, Hall PN, Wingate JP, Neoptolemos JP (1990) Evaluation of herniography in the diagnosis of an occult abdominal wall hernia in symptomatic adults. Br J Surg 77:902-906

124. Hallén M, Bergenfelz A, Westerdahl J (2008) Laparoscopic extraperitoneal inguinal hernia repair versus open mesh repair: long-term follow-up of a randomized controlled trial. Surgery 143:313-317

125. Halm EA, Lee C, Chassin MR (2002) Is volume related to outcome in health care? A systematic review and methodologic critique of the literature. Ann Intern Med 137:511-520

126. Hamilton EC, Scott DJ, Kapoor A, Nwariaku F, Bergen PC, Rege RV, Tesfay ST, Jones DB (2001) Improving operative performance using a laparoscopic hernia simulator. Am J Surg 182:725-728

127. Hamlin JA, Kahn AM (1998) Herniography: a review of 333 herniograms. Am Surg 64:965-969
128. Hamy A, Paineau J, Savigny JL, Vasse N, Visset J (1997) Sigmoid perforation, an exceptional late complication of peritoneal prosthesis for treatment of inguinal hernia. Int Surg 82:307-308

129. Heikkinen TJ, Haukipuro K, Hulkko A (1998) A cost and outcome comparison between laparoscopic and Lichtenstein hernia operations in a day-case unit. A randomized prospective study. Surg Endosc 12:1199-1203

130. Heikkinen T, Bringman S, Ohtonen P, Kunelius P, Haukipuro K, Hulkko A (2004) Five-year outcome of laparoscopic and Lichtenstein hernioplasties. Surg Endosc 18:518-522

131. Heikkinen T, Wollert S, Osterberg J, Smedberg S, Bringman S (2006) Early results of a randomised trial comparing Prolene and VyproII-mesh in endoscopic extraperitoneal inguinal hernia repair (TEP) of recurrent unilateral hernias. Hernia 10:34-40

132. Heise CP, Starling JR (1998) Mesh inguinodynia: a new clinical syndrome after inguinal herniorrhaphy? J Am Coll Surg 187:514-518

133. Heise CP, Sproat IA, Starling JR (2002) Peritoneography (herniography) for detecting occult inguinal hernia in patients with inguinodynia. Ann Surg 235:140-144

134. Herzog U (1990) Late results following inguinal or femoral hernia surgery. Langenbecks Arch Chir 375:5-10

135. Hofbauer C, Andersen PV, Juul P, Qvist N (1998) Late mesh rejection as a complication to transabdominal preperitoneal laparoscopic hernia repair. Surg Endosc 12:1164-1165

136. Højer AM, Rygaard H, Jess P (1997) CT in the diagnosis of abdominal wall hernias: a preliminary study. Eur Radiol 7:1416-1418

137. Horstmann R, Hellwig M, Classen C, Röttgermann S, Palmes D (2006) Impact of polypropylene amount on functional outcome and quality of life after inguinal hernia repair by the TAPP procedure using pure, mixed, and titanium-coated meshes. World J Surg 30:1742-1749

138. Hsia M, Ponsky L, Rosenblatt S, Jones JS (2004) Laparoscopic inguinal hernia repair complicates future pelvic oncologic surgery. Ann Surg 240:922-923

139. Hutter MM, Glasgow RE, Mulvihill SJ (2000) Does the participation of a surgical trainee adversely impact patient outcomes? A study of major pancreatic resections in California. Surgery 128:286-292

140. Ismail W, Taylor SJ, Beddow E (2000) Advice on driving after groin hernia surgery in the United Kingdom: questionnaire survey. BMJ 321:1056

141. Jain SK, Jayant M, Norbu C (2008) The role of antibiotic prophylaxis in mesh repair of primary inguinal hernias using prolene hernia system: a randomized prospective double-blind control trial. Trop Doct 38:80-82

142. Jarrett PE (2001) Day care surgery. Eur J Anaesthesiol Suppl 23:32-35

143. Jeans S, Williams GL, Stephenson BM (2007) Migration after open mesh plug inguinal hernioplasty: a review of the literature. Am Surg 73:207-209

144. Jensen P, Mikkelsen T, Kehlet H (2002) Postherniorrhaphy urinary retention-effect of local, regional, and general anesthesia: a review. Reg Anesth Pain Med 27:612-617

145. Jess P, Hauge C, Hansen CR (1999) Long-term results of repair of the internal ring for primary inguinal hernia. Eur J Surg 165:748-750

146. Jones RL, Wingate JP (1998) Herniography in the investigation of groin pain in adults. Clin Radiol 53:805-808

147. Jones KR, Burney RE, Peterson M, Christy B (2001) Return to work after inguinal hernia repair. Surgery 129:128-135

148. Kark A, Kurzer M, Waters KJ (1994) Accuracy of clinical diagnosis of direct and indirect inguinal hernia. Br J Surg 81:1081-1082 
149. Kark AE, Kurzer MN, Belsham PA (1998) Three thousand one hundred seventy-five primary inguinal hernia repairs: advantages of ambulatory open mesh repair using local anesthesia. J Am Coll Surg 186:447-455

150. Kehlet H, Bay Nielsen M (2005) Anaesthetic practice for groin hernia repair-a nation-wide study in Denmark 1998-2003. Acta Anaesthesiol Scand 49:143-146

151. Kendell J, Wildsmith JA, Gray IG (2000) Costing anaesthetic practice. An economic comparison of regional and general anaesthesia for varicose vein and inguinal hernia surgery. Anaesthesia 55:1106-1113

152. Kesek P, Ekberg O, Westlin N (2002) Herniographic findings in athletes with unclear groin pain. Acta Radiol 43:603-608

153. Khan LR, Kumar S, Nixon SJ (2006) Early results for new lightweight mesh in laparoscopic totally extra-peritoneal inguinal hernia repair. Hernia 10:303-308

154. Kingsnorth AN, Porter CS, Bennett DH, Walker AJ, Hyland ME, Sodergren S (2000) Lichtenstein patch or Perfix plug-andpatch in inguinal hernia: a prospective double-blind randomized controlled trial of short-term outcome. Surgery 127:276-283

155. Kingsnorth AN, Wright D, Porter CS, Robertson G (2002) Prolene Hernia System compared with Lichtenstein patch: a randomised double blind study of short-term and medium-term outcomes in primary inguinal hernia repair. Hernia 6:113-119

156. Klein SM, Pietrobon R, Nielsen KC, Steele SM, Warner DS, Moylan JA, Eubanks WS, Greengrass RA (2002) Paravertebral somatic nerve block compared with peripheral nerve blocks for outpatient inguinal herniorrhaphy. Reg Anesth Pain Med 27:476-480

157. Klinge U, Zheng H, Si ZY, Bhardwaj R, Klosterhalfen B, Schumpelick V (1999) Altered collagen synthesis in fascia transversalis of patients with inguinal hernia. Hernia 4:181-187

158. Knapp RW, Mullen JT (1976) Clinical evalation of the the of local anesthesia for repair of inguinal hernia. Am Surg 42:908910

159. Knook MT, Weidema WF, Stassen LP, Boelhouwer RU, van Steensel CJ (1999) Endoscopic totally extraperitoneal repair of bilateral inguinal hernias. Br J Surg 86:1312-1316

160. Knook MT, Weidema WF, Stassen LP, van Steensel CJ (1999) Endoscopic total extraperitoneal repair of primary and recurrent inguinal hernias. Surg Endosc 13:507-511

161. Knook MT, Weidema WF, Stassen LP, van Steensel CJ (1999) Laparoscopic repair of recurrent inguinal hernias after endoscopic herniorrhaphy. Surg Endosc 13:1145-1147

162. Koch A, Edwards A, Haapaniemi S, Nordin P, Kald A (2005) Prospective evaluation of 6895 groin hernia repairs in women. Br J Surg 92:1553-1558

163. Koch CA, Greenlee SM, Larson DR, Harrington JR, Farley DR (2006) Randomized prospective study of totally extraperitoneal inguinal hernia repair: fixation versus no fixation of mesh. JSLS 10:457-460

164. Koch CA, Grinberg GG, Farley DR (2006) Incidence and risk factors for urinary retention after endoscopic hernia repair. Am J Surg 191:381-385

165. Köninger J, Redecke J, Butters M (2004) Chronic pain after hernia repair: a randomized trial comparing Shouldice, Lichtenstein and TAPP. Langenbecks Arch Surg 389:361-365

166. Kraft BM, Kolb H, Kuckuk B, Haaga S, Leibl BJ, Kraft K, Bittner R (2003) Diagnosis and classification of inguinal hernias. Surg Endosc 17:2021-2024

167. Kugel RD (1999) Minimally invasive, nonlaparoscopic, preperitoneal, and sutureless, inguinal herniorrhaphy. Am J Surg 178:298-302

168. Kuhry E, van Veen RN, Langeveld HR, Steyerberg EW, Jeekel J, Bonjer HJ (2007) Open or endoscopic total extraperitoneal inguinal hernia repair? A systematic review. Surg Endosc 21:161-166

169. Kulah B, Duzgun AP, Moran M, Kulacoglu IH, Ozmen MM, Coskun F (2001) Emergency hernia repairs in elderly patients. Am J Surg 182:455-459

170. Kulah B, Kulacoglu IH, Oruc MT, Duzgun AP, Moran M, Ozmen MM, Coskun F (2001) Presentation and outcome of incarcerated external hernias in adults. Am J Surg 181:101-104

171. Langenbach MR, Schmidt J, Zirngibl H (2003) Comparison of biomaterials in the early postoperative period. Surg Endosc 17:1105-1109

172. Langenbach MR, Schmidt J, Zirngibl H (2006) Comparison of biomaterials: three meshes and TAPP for inguinal hernia. Surg Endosc 20:1511-1517

173. Lau H (2005) Fibrin sealant versus mechanical stapling for mesh fixation during endoscopic extraperitoneal inguinal hernioplasty: a randomized prospective trial. Ann Surg 242:670-675

174. Lau H, Patil NG, Yuen WK, Lee F (2002) Learning curve for unilateral endoscopic totally extraperitoneal (TEP) inguinal hernioplasty. Surg Endosc 16:1724-1728

175. Lau H, Patil NG, Yuen WK, Lee F (2002) Urinary retention following endoscopic totally extraperitoneal inguinal hernioplasty. Surg Endosc 16:1547-1550

176. Lau H, Patil NG, Yuen WK (2006) Day-case endoscopic totally extraperitoneal inguinal hernioplasty versus open Lichtenstein hernioplasty for unilateral primary inguinal hernia in males: a randomized trial. Surg Endosc 20:76-81

177. Lau H, Fang C, Yuen WK, Patil NG (2007) Risk factors for inguinal hernia in adult males: a case-control study. Surgery 141:262-266

178. Lazorthes F, Chiotasso P, Massip P, Materre JP, Sarkissian M (1992) Local antibiotic prophylaxis in inguinal hernia repair. Surg Gynecol Obstet 175:569-570

179. Leander P, Ekberg O, Sjöberg S, Kesek P (2000) MR imaging following herniography in patients with unclear groin pain. Eur Radiol 10:1691-1696

180. LeBlanc KA (2001) Complications associated with the plugand-patch method of inguinal herniorrhaphy. Hernia 5:135-138

181. Lehnert B, Wadouh F (1992) High coincidence of inguinal hernias and abdominal aortic aneurysms. Ann Vasc Surg 6:134137

182. Leibl BJ, Däubler P, Schmedt CG, Kraft K, Bittner R (2000) Long-term results of a randomized clinical trial between laparoscopic hernioplasty and shouldice repair. Br J Surg 87:780783

183. Lichtenstein IL, Shulman AG, Amid PK, Montllor MM (1989) The tension-free hernioplasty. Am J Surg 157:188-193

184. Liem MS, van Steensel CJ, Boelhouwer RU, Weidema WF, Clevers GJ, Meijer WS, Vente JP, de Vries LS, van Vroonhoven TJ (1996) The learning curve for totally extraperitoneal laparoscopic inguinal hernia repair. Am J Surg 171:281-285

185. Liem MS, Halsema JA, van der Graaf Y, Schrijvers AJ, van Vroonhoven TJ (1997) Cost-effectiveness of extraperitoneal laparoscopic inguinal hernia repair: a randomized comparison with conventional herniorrhaphy. Coala trial group. Ann Surg 226:668-675

186. Liem MS, van der Graaf Y, van Steensel CJ, Boelhouwer RU, Clevers GJ, Meijer WS, Stassen LP, Vente JP, Weidema WF, Schrijvers AJ, van Vroonhoven TJ (1997) Comparison of conventional anterior surgery and laparoscopic surgery for inguinalhernia repair. N Engl J Med 336:1541-1547

187. Liem MS, van der Graaf Y, Zwart RC, Geurts I, van Vroonhoven TJ (1997) A randomized comparison of physical performance following laparoscopic and open inguinal hernia repair. The Coala Trial Group. Br J Surg 84:64-67 
188. Liem MS, van der Graaf Y, Zwart RC, Geurts I, van Vroonhoven TJ (1997) Risk factors for inguinal hernia in women: a case-control study. The Coala Trial Group. Am J Epidemiol 146:721-726

189. Lilly MC, Arregui ME (2002) Ultrasound of the inguinal floor for evaluation of hernias. Surg Endosc 16:659-662

190. Lodding P, Bergdahl C, Nyberg M, Pileblad E, Stranne J, Hugosson J (2001) Inguinal hernia after radical retropubic prostatectomy for prostate cancer: a study of incidence and risk factors in comparison to no operation and lymphadenectomy. J Urol 166:964-967

191. Loftus IM, Ubhi SS, Rodgers PM, Watkin DF (1997) A negative herniogram does not exclude the presence of a hernia. Ann R Coll Surg Engl 79:372-375

192. Lovisetto F, Zonta S, Rota E, Bottero L, Faillace G, Turra G, Fantini A, Longoni M (2007) Laparoscopic transabdominal preperitoneal (TAPP) hernia repair: surgical phases and complications. Surg Endosc 21:646-652

193. MacFadyen BV Jr, Mathis CR (1994) Inguinal herniorrhaphy: complications and recurrences. Semin Laparosc Surg 1:128-140

194. MacFadyen BV Jr, Arregui ME, Corbitt JD Jr, Filipi CJ, Fitzgibbons RJ Jr, Franklin ME, McKernan JB, Olsen DO, Phillips EH, Rosenthal D, Schultz LS, Sewell RW, Smoot RT, Spaw AT, Toy FK, Waddell RL, Zucker KA (1993) Complications of laparoscopic herniorrhaphy. Surg Endosc 7:155-158

195. Mahon D, Decadt B, Rhodes M (2003) Prospective randomized trial of laparoscopic (transabdominal preperitoneal) vs open (mesh) repair for bilateral and recurrent inguinal hernia. Surg Endosc 17:1386-1390

196. Malek S, Torella F, Edwards PR (2004) Emergency repair of groin herniae: outcome and implications for elective surgery waiting times. Int J Clin Pract 58:207-209

197. McCormack K, Scott NW, Go PM, Ross S, Grant AM; EU Hernia Trialists Collaboration (2003) Laparoscopic techniques versus open techniques for inguinal hernia repair. Cochrane Database Syst Rev CD001785

198. McCormack K, Wake B, Perez J, Fraser C, Cook J, McIntosh E, Vale L, Grant A (2005) Laparoscopic surgery for inguinal hernia repair: systematic review of effectiveness and economic evaluation. Health Technol Assess 9:1-203; iii-iv

199. McCormack K, Wake BL, Fraser C, Vale L, Perez J, Grant A (2005) Transabdominal pre-peritoneal (TAPP) versus totally extraperitoneal (TEP) laparoscopic techniques for inguinal hernia repair: a systematic review. Hernia 9:109-114

200. McEntee GP, O'Carroll A, Mooney B, Egan TJ, Delaney PV (1989) Timing of strangulation in adult hernias. Br J Surg 76:725-726

201. McGillicuddy JE (1998) Prospective randomized comparison of the Shouldice and Lichtenstein hernia repair procedures. Arch Surg 133:974-978

202. McIntosh A, Hutchinson A, Roberts A, Withers H (2000) Evidence-based management of groin hernia in primary care-a systematic review. Fam Pract 17:442-447

203. Memon MA, Cooper NJ, Memon B, Memon MI, Abrams KR (2003) Meta-analysis of randomized clinical trials comparing open and laparoscopic inguinal hernia repair. $\mathrm{Br} \mathrm{J}$ Surg 90:1479-1492

204. Michelsen M, Walter F (1982) Comparison of outpatient and inpatient operations for inguinal hernia (1971 to 1978) (author's transl). Zentralbl Chir 107:94-102

205. Miedema BW, Ibrahim SM, Davis BD, Koivunen DG (2004) A prospective trial of primary inguinal hernia repair by surgical trainees. Hernia 8:28-32

206. Millat B; Fédération de Recherche EN CHirurgie (FRENCH) (2007) Inguinal hernia repair. A randomized multicentric study comparing laparoscopic and open surgical repair. J Chir (Paris) 144:119-124

207. Miserez M (2004) Inguinal hernia repair in general surgical practice in 2004: more than laparoscopy, training is the keyword. Acta Chir Belg 104:422-424

208. Miserez M, Alexandre JH, Campanelli G, Corcione F, Cuccurullo D, Pascual MH, Hoeferlin A, Kingsnorth AN, Mandala V, Palot JP, Schumpelick V, Simmermacher RK, Stoppa R, Flament JB (2007) The European hernia society groin hernia classification: simple and easy to remember. Hernia 11:113-116

209. Mitchell JB, Harrow B (1994) Costs and outcomes of inpatient versus outpatient hernia repair. Health Policy 28:143-152

210. Morales R, Carmona A, Pagán A (2000) Utility of antibiotic prophylaxis in reducing wound infection in inguinal or femoral hernia repair using polypropylene mesh. Cir Esp 67:51-59

211. Moreno-Egea A, Torralba Martínez JA, Morales Cuenca G, Aguayo Albasini JL (2004) Randomized clinical trial of fixation vs nonfixation of mesh in total extraperitoneal inguinal hernioplasty. Arch Surg 139:1376-1379

212. Nadkarni S, Brown PW, van Beek EJ, Collins MC (2001) Herniography: a prospective, randomized study between midline and left iliac fossa puncture techniques. Clin Radiol 56:389-392

213. Negro P, Gossetti F, D'Amore L, Proposito D, Vermeil V, Battillocchi B, Della Rocca G, Carboni M (2000) 1,000 prosthetic hernia repairs: experience of a dedicated team. Chir Ital $52: 279-288$

214. Neuhauser D (1997) Elective inguinal herniorrhaphy versus a truss in the elderly. In: Bunker JP, Barnes BA, Mosteller F (eds) Costs, risks and benefits of surgery. Oxford University Press, New York

215. Neumayer L, Giobbie-Hurder A, Jonasson O, Fitzgibbons R Jr, Dunlop D, Gibbs J, Reda D, Henderson W; Veterans Affairs Cooperative Studies Program 456 Investigators (2004) Open mesh versus laparoscopic mesh repair of inguinal hernia. $\mathrm{N}$ Engl J Med 350:1819-1827

216. Neumayer LA, Gawande AA, Wang J, Giobbie-Hurder A, Itani KM, Fitzgibbons RJ Jr, Reda D, Jonasson O; CSP \#456 Investigators (2005) Proficiency of surgeons in inguinal hernia repair: effect of experience and age. Ann Surg 242:344-348

217. Nienhuijs SW, Boelens OB, Strobbe LJ (2005) Pain after anterior mesh hernia repair. J Am Coll Surg 200:885-889

218. Nienhuijs SW, van Oort I, Keemers-Gels ME, Strobbe LJ, Rosman C (2005) Randomized trial comparing the Prolene Hernia System, mesh plug repair and Lichtenstein method for open inguinal hernia repair. Br J Surg 92:33-38

219. Nilsson H, Stylianidis G, Haapamäki M, Nilsson E, Nordin P (2007) Mortality after groin hernia surgery. Ann Surg 245:656660

220. Nordin P, Bartelmess P, Jansson C, Svensson C, Edlund G (2002) Randomized trial of Lichtenstein versus Shouldice hernia repair in general surgical practice. Br J Surg 89:45-49

221. Nordin P, Zetterström H, Gunnarsson U, Nilsson E (2003) Local, regional, or general anaesthesia in groin hernia repair: multicentre randomised trial. Lancet 362:853-858

222. Nordin P, Hernell H, Unosson M, Gunnarsson U, Nilsson E (2004) Type of anaesthesia and patient acceptance in groin hernia repair: a multicentre randomised trial. Hernia 8:220-225

223. Nordin P, Zetterström H, Carlsson P, Nilsson E (2007) Costeffectiveness analysis of local, regional and general anaesthesia for inguinal hernia repair using data from a randomized clinical trial. Br J Surg 94:500-505

224. Nyhus LM (2004) Classification of groin hernia: milestones. Hernia 8:87-88

225. O'Dwyer PJ (2004) Current status of the debate on laparoscopic hernia repair. Br Med Bull 70:105-118 
226. O’Dwyer PJ, Chung L (2006) Watchful waiting was as safe as surgical repair for minimally symptomatic inguinal hernias. Evid Based Med 11:73

227. O’Dwyer PJ, Serpell MG, Millar K, Paterson C, Young D, Hair A, Courtney CA, Horgan P, Kumar S, Walker A, Ford I (2003) Local or general anesthesia for open hernia repair: a randomized trial. Ann Surg 237:574-579

228. O'Dwyer PJ, Kingsnorth AN, Molloy RG, Small PK, Lammers B, Horeyseck G (2005) Randomized clinical trial assessing impact of a lightweight or heavyweight mesh on chronic pain after inguinal hernia repair. Br J Surg 92:166-170

229. Ojo P, Abenthroth A, Fiedler P, Yavorek G (2006) Migrating mesh mimicking colonic malignancy. Am Surg 72:1210-1211

230. Oteiza F, Ciga MA, Ortiz H (2004) Antibiotic prophylaxis in inguinal herniaplasty. Cir Esp 75:69-71

231. Ozgün H, Kurt MN, Kurt I, Cevikel MH (2002) Comparison of local, spinal, and general anaesthesia for inguinal herniorrhaphy. Eur J Surg 168:455-459

232. Pascual JB, Baranda MM, Tarrero MT, Gutiérrez FM, Garrido IM, Errasti CA (1990) Subcutaneous emphysema, pneumomediastinum, bilateral pneumothorax and pneumopericardium after laparoscopy. Endoscopy 22:59

233. Payne JH Jr, Grininger LM, Izawa MT, Podoll EF, Lindahl PJ, Balfour J (1994) Laparoscopic or open inguinal herniorrhaphy? A randomized prospective trial. Arch Surg 129:973-979

234. Peiper C, Conze J, Ponschek N, Schumpelick V (1997) Value of subcutaneous drainage in repair of primary inguinal hernia. A prospective randomized study of 100 cases. Chirurg 68:6367

235. Perez AR, Roxas MF, Hilvano SS (2005) A randomized, doubleblind, placebo-controlled trial to determine effectiveness of antibiotic prophylaxis for tension-free mesh herniorrhaphy. J Am Coll Surg 200:393-397

236. Phillips EH, Arregui M, Carroll BJ, Corbitt J, Crafton WB, Fallas MJ, Filipi C, Fitzgibbons RJ, Franklin MJ, McKernan B, Olsen D, Ortega A, Payne JH Jr, Peters J, Rodriguez R, Rosette P, Schultz L, Seid A, Sewell R, Smoot R, Toy F, Waddell R, Watson $S$ (1995) Incidence of complications following laparoscopic hernioplasty. Surg Endosc 9:16-21

237. Pineault R, Contandriopoulos AP, Valois M, Bastian ML, Lance JM (1985) Randomized clinical trial of one-day surgery. Patient satisfaction, clinical outcomes, and costs. Med Care 23:171-182

238. Platt R, Zaleznik DF, Hopkins CC, Dellinger EP, Karchmer AW, Bryan CS, Burke JF, Wikler MA, Marino SK, Holbrook KF, Tosteson TD, Segal MR (1990) Perioperative antibiotic prophylaxis for herniorrhaphy and breast surgery. N Engl J Med 322:153-160

239. Pleumeekers HJ, De Gruijl A, Hofman A, Van Beek AJ, Hoes AW (1999) Prevalence of aortic aneurysm in men with a history of inguinal hernia repair. Br J Surg 86:1155-1158

240. Pokorny H, Klingler A, Schmid T, Fortelny R, Hollinsky C, Kawji R, Steiner E, Pernthaler H, Függer R, Scheyer M (2008) Recurrence and complications after laparoscopic versus open inguinal hernia repair: results of a prospective randomized multicenter trial. Hernia 12:385-389

241. Ponka JL (1980) Hernias of the abdominal wall. WB Saunders, Philadelphia

242. Poobalan AS, Bruce J, Smith WC, King PM, Krukowski ZH, Chambers WA (2003) A review of chronic pain after inguinal herniorrhaphy. Clin J Pain 19:48-54

243. Post S (1997) Against the principle surgical indications in inguinal hernia. Chirurg 68:1251-1255

244. Post S, Weiss B, Willer M, Neufang T, Lorenz D (2004) Randomized clinical trial of lightweight composite mesh for Lichtenstein inguinal hernia repair. Br J Surg 91:44-48
245. Prabhu A, Chung F (2001) Anaesthetic strategies towards developments in day care surgery. Eur J Anaesthesiol Suppl 23:36-42

246. Praseedom RK, Paisley A, Madhavan KK, Garden OJ, Carter DC, Paterson-Brown S (1999) Supervised surgical trainees can perform pancreatic resections safely. J R Coll Surg Edinb 44:16-18

247. Prescott RJ, Cutherbertson C, Fenwick N, Garraway WM, Ruckley CV (1978) Economic aspects of day care after operations for hernia or varicose veins. J Epidemiol Community Health 32:222-225

248. Primatesta P, Goldacre MJ (1996) Inguinal hernia repair: incidence of elective and emergency surgery, readmission and mortality. Int J Epidemiol 25:835-839

249. Prismant (2000) Jaardiskette voor ziekenhuizen 1999-2000

250. Quill DS, Devlin HB, Plant JA, Denham KR, McNay RA, Morris D (1983) Surgical operation rates: a twelve year experience in Stockton on Tees. Ann R Coll Surg Engl 65:248-253

251. Rai S, Chandra SS, Smile SR (1998) A study of the risk of strangulation and obstruction in groin hernias. Aust N Z J Surg 68:650-654

252. Ralphs DN, Brain AJ, Grundy DJ, Hobsley M (1980) How accurately can direct and indirect inguinal hernias be distinguished? Br Med J 280:1039-1040

253. Ramia JM, Pardo R, Cubo T, Padilla D, Hernández-Calvo J (1999) Pneumomediastinum as a complication of extraperitoneal laparoscopic inguinal hernia repair. JSLS 3:233-234

254. Ramsay CR, Grant AM, Wallace SA, Garthwaite PH, Monk AF, Russell IT (2001) Statistical assessment of the learning curves of health technologies. Health Technol Assess 5:1-79

255. Ramyil VM, Ognonna BC, Iya D (1999) Patient acceptance of outpatient treatment for inguinal hernia in Jos, Nigeria. Cent Afr J Med 45:244-246

256. Read RC, Barone GW, Hauer-Jensen M, Yoder G (1993) Properitoneal prosthetic placement through the groin. The anterior (Mahorner-Goss, Rives-Stoppa) approach. Surg Clin North Am 73:545-555

257. Reid I, Devlin HB (1994) Testicular atrophy as a consequence of inguinal hernia repair. Br J Surg 81:91-93

258. Rider MA, Baker DM, Locker A, Fawcett AN (1993) Return to work after inguinal hernia repair. Br J Surg 80:745-746

259. Ris HB, Aebersold P, Küpfer K, Stucki U, Stirnemann H, Doran J (1987) 10 years' experience using a modified Shouldice surgical technic for inguinal hernia in adults. II. Which factors modify the recurrence of inguinal hernia? Chirurg 58:100-105

260. Robinson P, Hensor E, Lansdown MJ, Ambrose NS, Chapman $\mathrm{AH}$ (2006) Inguinofemoral hernia: accuracy of sonography in patients with indeterminate clinical features. AJR Am J Roentgenol 187:1168-1178

261. Robson AJ, Wallace CG, Sharma AK, Nixon SJ, PatersonBrown S (2004) Effects of training and supervision on recurrence rate after inguinal hernia repair. Br J Surg 91:774-777

262. Ruckley CV, Cuthbertson C, Fenwick N, Prescott RJ, Garraway WM (1978) Day care after operations for hernia or varicose veins: a controlled trial. Br J Surg 65:456-459

263. Rutkow IM (1998) Epidemiologic, economic, and sociologic aspects of hernia surgery in the United States in the 1990s. Surg Clin North Am 78:941-951; v-vi

264. Ryan JA Jr, Adye BA, Jolly PC, Mulroy MF 2nd (1984) Outpatient inguinal herniorrhaphy with both regional and local anesthesia. Am J Surg 148:313-316

265. Sakorafas GH, Halikias I, Nissotakis C, Kotsifopoulos N, Stavrou A, Antonopoulos C, Kassaras GA (2001) Open tension free repair of inguinal hernias; the Lichtenstein technique. BMC Surg 1:3 
266. Salcedo-Wasicek MC, Thirlby RC (1995) Postoperative course after inguinal herniorrhaphy. A case-controlled comparison of patients receiving workers' compensation vs patients with commercial insurance. Arch Surg 130:29-32

267. Sanabria A, Domínguez LC, Valdivieso E, Gómez G (2007) Prophylactic antibiotics for mesh inguinal hernioplasty: a metaanalysis. Ann Surg 245:392-396

268. Sanchez-Manuel FJ, Seco-Gil JL (2004) Antibiotic prophylaxis for hernia repair. Cochrane Database Syst Rev 4:CD003769

269. Sandbichler P, Gstir H, Baumgartner C, Furtschegger A, Egender G, Steiner E (1994) Laparoscopic surgery of inguinal hernia by transperitoneal implantation of an artificial mesh. Technique and early results. Chirurg 65:64-67

270. Sanjay P, Harris D, Jones P, Woodward A (2006) Randomized controlled trial comparing prolene hernia system and lichtenstein method for inguinal hernia repair. ANZ J Surg 76:548-552

271. Schmedt CG, Leibl BJ, Bittner R (2002) Endoscopic inguinal hernia repair in comparison with Shouldice and Lichtenstein repair. A systematic review of randomized trials. Dig Surg 19:511-517

272. Schmedt CG, Sauerland S, Bittner R (2005) Comparison of endoscopic procedures vs Lichtenstein and other open mesh techniques for inguinal hernia repair: a meta-analysis of randomized controlled trials. Surg Endosc 19:188-199

273. Schmitz R, Shah S, Treckmann J, Schneider K (1997) Extraperitoneal, "tension free" inguinal hernia repair with local anesthesia-a contribution to effectiveness and economy. Langenbecks Arch Chir Suppl Kongressbd 114:1135-1138

274. Schwetling R, Bärlehner E (1998) Is there an indication for general perioperative antibiotic prophylaxis in laparoscopic plastic hernia repair with implantation of alloplastic tissue? Zentralbl Chir 123:193-195

275. Scott NW, McCormack K, Graham P, Go PM, Ross SJ, Grant AM (2002) Open mesh versus non-mesh for repair of femoral and inguinal hernia. Cochrane Database Syst Rev CD002197

276. Shaked A, Calderom I, Durst A (1991) Safety of surgical procedures performed by residents. Arch Surg 126:559-560

277. Shulman AG, Amid PK, Lichtenstein IL (1995) A survey of non-expert surgeons using the open tension-free mesh patch repair for primary inguinal hernias. Int Surg 80:35-36

278. Simons MP, Obertop H (2001) Lichamelijk onderzoek geeft uitsluitsel over een mediale dan wel een laterale liesbreuk. In: van Everdingen JJE (ed) Medische misvattingen. Bohn Stafleu Van Loghum, Houten

279. Simons MP, Kleijnen J, van Geldere D, Hoitsma HF, Obertop H (1996) Role of the Shouldice technique in inguinal hernia repair: a systematic review of controlled trials and a meta-analysis. Br J Surg 83:734-738

280. Smietański M, Renke M, Bigda J, Smietańska I, Rutkowski B, Witkowski P, Sledziński Z (2006) Management of inguinal hernia on peritoneal dialysis: an audit of current Polish practice and call for a standard. Int J Artif Organs 29:573-577

281. Smith AI, Royston CM, Sedman PC (1999) Stapled and nonstapled laparoscopic transabdominal preperitoneal (TAPP) inguinal hernia repair. A prospective randomized trial. Surg Endosc 13:804-806

282. Song D, Greilich NB, White PF, Watcha MF, Tongier WK (2000) Recovery profiles and costs of anesthesia for outpatient unilateral inguinal herniorrhaphy. Anesth Analg 91:876-881

283. Sorensen LT, Friis E, Jorgensen T, Vennits B, Andersen BR, Rasmussen GI, Kjaergaard J (2002) Smoking is a risk factor for recurrence of groin hernia. World J Surg 26:397-400

284. Sparkman RS (1962) Bilateral exploration in inguinal hernia in juvenile patients. Review and appraisal. Surgery 51:393-406

285. Stengel D, Bauwens K, Ekkernkamp A (2004) Recurrence risks in randomized trials of laparoscopic versus open inguinal hernia repair: to pool or not to pool (this is not the question). Langenbecks Arch Surg 389:492-498

286. Stoppa RE, Rives JL, Warlaumont CR, Palot JP, Verhaeghe PJ, Delattre JF (1984) The use of Dacron in the repair of hernias of the groin. Surg Clin North Am 64:269-285

287. Stranne J, Hugosson J, Iversen P, Morris T, Lodding P (2005) Inguinal hernia in stage M0 prostate cancer: a comparison of incidence in men treated with and without radical retropubic prostatectomy — an analysis of 1105 patients. Urology 65:847851

288. Stranne J, Hugosson J, Lodding P (2006) Post-radical retropubic prostatectomy inguinal hernia: an analysis of risk factors with special reference to preoperative inguinal hernia morbidity and pelvic lymph node dissection. J Urol 176:2072-2076

289. Stranne J, Hugosson J, Lodding P (2007) Inguinal hernia is a common complication in lower midline incision surgery. Hernia $11: 247-252$

290. Sultana A, Jagdish S, Pai D, Rajendiran KM (1999) Inguinal herniorrhaphy under local anaesthesia and spinal anaesthesia-a comparative study. J Indian Med Assoc 97:169-170, 175

291. Tamme C, Scheidbach H, Hampe C, Schneider C, Köckerling F (2003) Totally extraperitoneal endoscopic inguinal hernia repair (TEP). Surg Endosc 17:190-195

292. Taylor EW, Dewar EP (1983) Early return to work after repair of a unilateral inguinal hernia. Br J Surg 70:599-600

293. Taylor SG, O’Dwyer PJ (1999) Chronic groin sepsis following tension-free inguinal hernioplasty. Br J Surg 86:562-565

294. Taylor EW, Byrne DJ, Leaper DJ, Karran SJ, Browne MK, Mitchell KJ (1997) Antibiotic prophylaxis and open groin hernia repair. World J Surg 21:811-814

295. Teasdale C, McCrum AM, Williams NB, Horton RE (1982) A randomised controlled trial to compare local with general anaesthesia for short-stay inguinal hernia repair. Ann R Coll Surg Engl 64:238-242

296. The MRC Laparoscopic Groin Hernia Trial Group (1999) Laparoscopic versus open repair of groin hernia: a randomised comparison. Lancet 354:185-190

297. Tobin GR, Clark S, Peacock EE Jr (1976) A neuromuscular basis for development of indirect inguinal hernia. Arch Surg 111:464-466

298. Tocchi A, Liotta G, Mazzoni G, Lepre L, Costa G, Maggiolini F, Miccini M (1998) Learning curve for "tension-free" reparation of inguinal hernia. G Chir 19:199-203

299. Truong S, Pfingsten FP, Dreuw B, Schumpelick V (1993) Value of sonography in diagnosis of uncertain lesions of the abdominal wall and inguinal region. Chirurg 64:468-475

300. Tschudi JF, Wagner M, Klaiber C, Brugger JJ, Frei E, Krähenbühl L, Inderbitzi R, Boinski J, Hsu Schmitz SF, Hüsler J (2001) Randomized controlled trial of laparoscopic transabdominal preperitoneal hernioplasty vs Shouldice repair. Surg Endosc 15:1263-1266

301. Tucker JG, Wilson RA, Ramshaw BJ, Mason EM, Duncan TD, Lucas GW (1995) Laparoscopic herniorrhaphy: technical concerns in prevention of complications and early recurrence. Am Surg 61:36-39

302. Tverskoy M, Cozacov C, Ayache M, Bradley EL Jr, Kissin I (1990) Postoperative pain after inguinal herniorrhaphy with different types of anesthesia. Anesth Analg 70:29-35

303. Tzovaras G, Delikoukos S, Christodoulides G, Spyridakis M, Mantzos F, Tepetes K, Athanassiou E, Hatzitheofilou C (2007) The role of antibiotic prophylaxis in elective tension-free mesh inguinal hernia repair: results of a single-centre prospective randomised trial. Int J Clin Pract 61:236-239

304. Vale L, McCormack K, Scott N, Grant A (2000) Systematic review of the effectiveness and cost-effectiveness of laparoscopic versus open repair of inguinal hernia. Health Services 
Research Unit, Health Economics Research Unit, University of Aberdeen

305. Vale L, Ludbrook A, Grant A (2003) Assessing the costs and consequences of laparoscopic vs. open methods of groin hernia repair: a systematic review. Surg Endosc 17:844-849

306. van den Berg JC, de Valois JC, Go PM, Rosenbusch G (1999) Detection of groin hernia with physical examination, ultrasound, and MRI compared with laparoscopic findings. Invest Radiol 34:739-743

307. van den Berg JC, de Valois JC, Go PM, Rosenbusch G (2000) Radiological anatomy of the groin region. Eur Radiol 10:661670

308. van Veen RN, van Wessem KJ, Halm JA, Simons MP, Plaisier PW, Jeekel J, Lange JF (2007) Patent processus vaginalis in the adult as a risk factor for the occurrence of indirect inguinal hernia. Surg Endosc 21:202-205

309. van Veen RN, Wijsmuller AR, Vrijland WW, Hop WC, Lange JF, Jeekel J (2007) Long-term follow-up of a randomized clinical trial of non-mesh versus mesh repair of primary inguinal hernia. Br J Surg 94:506-510

310. van Veen RN, Mahabier C, Dawson I, Hop WC, Kok NF, Lange JF, Jeekel J (2008) Spinal or local anesthesia in lichtenstein hernia repair: a randomized controlled trial. Ann Surg 247:428433

311. Velanovich V, Shadduck P, Khaitan L, Morton J, Maupin G, Traverso LW (2006) Analysis of the SAGES Outcomes Initiative groin hernia database. Surg Endosc 20:191-198

312. Voitk AJ (1998) The learning curve in laparoscopic inguinal hernia repair for the community general surgeon. Can J Surg 41:446-450

313. Wake BL, McCormack K, Fraser C, Vale L, Perez J, Grant AM (2005) Transabdominal pre-peritoneal (TAPP) vs totally extraperitoneal (TEP) laparoscopic techniques for inguinal hernia repair. Cochrane Database Syst Rev CD004703

314. Wantz GE (1989) Giant prosthetic reinforcement of the visceral sac. Surg Gynecol Obstet 169:408-417

315. Wantz GE (1993) Testicular atrophy and chronic residual neuralgia as risks of inguinal hernioplasty. Surg Clin North Am 73:571-581
316. Wellwood J, Sculpher MJ, Stoker D, Nicholls GJ, Geddes C, Whitehead A, Singh R, Spiegelhalter D (1998) Randomised controlled trial of laparoscopic versus open mesh repair for inguinal hernia: outcome and cost. BMJ 317:103-110

317. Weyhe D, Winnemöller C, Hellwig A, Meurer K, Plugge H, Kasoly K, Laubenthal H, Bauer KH, Uhl W (2006) (section sign) $115 \mathrm{~b}$ SGB $\mathrm{V}$ threatens outpatient treatment for inguinal hernia. Analysis of outcome and economics. Chirurg 77:844855

318. Wijsmuller AR, van Veen RN, Bosch JL, Lange JF, Kleinrensink GJ, Jeekel J, Lange JF (2007) Nerve management during open hernia repair. Br J Surg 94:17-22

319. Wilkiemeyer M, Pappas TN, Giobbie-Hurder A, Itani KM, Jonasson O, Neumayer LA (2005) Does resident post graduate year influence the outcomes of inguinal hernia repair? Ann Surg 241:879-882

320. Wilson MS, Irving SO, Iddon J, Deans GT, Brough WA (1998) A measurement of the ability to drive after different types of inguinal hernia repair. Surg Laparosc Endosc 8:384-387

321. Wright D, Paterson C, Scott N, Hair A, O'Dwyer PJ (2002) Five-year follow-up of patients undergoing laparoscopic or open groin hernia repair: a randomized controlled trial. Ann Surg 235:333-337

322. Yerdel MA, Akin EB, Dolalan S, Turkcapar AG, Pehlivan M, Gecim IE, Kuterdem E (2001) Effect of single-dose prophylactic ampicillin and sulbactam on wound infection after tension-free inguinal hernia repair with polypropylene mesh: the randomized, double-blind, prospective trial. Ann Surg 233:26-33

323. Zieren J, Küpper F, Paul M, Neuss H, Müller JM (2003) Inguinal hernia: obligatory indication for elective surgery? A prospective assessment of quality of life before and after plug and patch inguinal hernia repair. Langenbecks Arch Surg 387:417-420

324. Zollinger RM Jr (2003) Classification systems for groin hernias. Surg Clin North Am 83:1053-1063 